University of Tennessee Health Science Center UTHSC Digital Commons

\title{
The Role of Secretory Phospholipase A2 Group IIA in Obesity and Metabolism
}

\author{
Michael S. Kuefner \\ University of Tennessee Health Science Center
}

Follow this and additional works at: https://dc.uthsc.edu/dissertations

Part of the Medicine and Health Sciences Commons, and the Pharmacology Commons

\section{Recommended Citation}

Kuefner, Michael S. (0000-0002-2965-365X), "The Role of Secretory Phospholipase A2 Group IIA in Obesity and Metabolism" (2019). Theses and Dissertations (ETD). Paper 496. http://dx.doi.org/10.21007/ etd.cghs.2019.0581.

This Dissertation is brought to you for free and open access by the College of Graduate Health Sciences at UTHSC Digital Commons. It has been accepted for inclusion in Theses and Dissertations (ETD) by an authorized administrator of UTHSC Digital Commons. For more information, please contact jwelch30@uthsc.edu. 


\title{
The Role of Secretory Phospholipase A2 Group IIA in Obesity and Metabolism
}

\begin{abstract}
Secretory phospholipase A2 group IIA (PLA2G2A) is a member of a family of secretory phospholipases previously implicated in inflammation, atherogenesis, and antibacterial actions. These enzymes hydrolyze glycerophospholipids at the sn-2 position releasing lysophospholipids and fatty acids. Though studies have shown PLA2G2A is pro-inflammatory and promotes atherosclerosis, no research has analyzed the role of this enzyme in obesity and metabolism. Studies in the past 5-10 years utilizing various knock- out or over-expression mouse models have analyzed the role of different secretory phospholipase A2s (sPLA2) in metabolic diseases. From these studies, it is known that at least seven of the $11 \mathrm{sPLA} 2$ isozymes contribute to the prevention or causation of metabolic diseases such as obesity and Type 2 diabetes. Increased energy expenditure, insulin sensitivity, and glucose tolerance are common characteristics of a mouse with a beneficial metabolic phenotype. We studied the impact of a high-fat diet on wild-type C57BL/6 and C57BL/6 mice over-expressing the human PLA2G2A gene (IIA+ mice). Despite no differences in food intake, the IIA+ mice weighed less, had increased oxygen consumption and energy expenditure, increased glucose tolerance and insulin sensitivity, and significantly less fat mass. There was a significant induction of uncoupling protein 1 (UCP1) in the brown adipose tissue (BAT) of IIA+ mice, suggesting enhanced mitochondrial uncoupling in their adipose tissue to drive this metabolic phenotype. Previous work in Dr. Park's laboratory suggested that hepatic PLA2G2A expression is negatively regulated by acute thyroid hormone administration. We also examined the regulation of PLA2G2A and the metabolic changes that occur in response to variations in thyroid status. The impact of PLA2G2A on brown adipose tissue thermogenic gene expression was explored. C57BL/6 and IIA+ mice were made hypothyroid over a 10-week period or treated with thyroid hormone (T3) for 5 weeks. There were no significant changes in PLA2G2A abundance in response to thyroid status. The hypothyroid IIA+ mice did not increase their energy expenditure even with T3 administration. However, the energy expenditure, substrate utilization, insulin sensitivity and glucose tolerance were all elevated in the IIA+ mice given T3. Moreover, white adipocytes from IIA+ mice were much more prone to 'beiging,' including increased expression of brown adipose thermogenic markers such as uncoupling protein 1 (UCP1), PR domain containing 16 (PRDM-16), and early B cell factor 2 (EBF2). Finally, the brown adipose tissue of IIA+ mice had increased UCP1. Further metabolic analysis of C57BL/ 6 and IIA+ mice revealed that even at thermoneutrality the IIA+ mice display elevated oxygen consumption and energy expenditure. Moreover, citrate synthase activity was increased in the brown adipose tissue but not muscle in IIA+ animals, suggesting that the elevated thermogenesis is adipose tissue-specific. Finally, RNA microarray analysis of brown adipose tissue from $\mathrm{C} 57 \mathrm{BL} / 6$ and IIA+ mice showed induction of numerous genes related to fuel substrate transport and metabolism, indicating an increase in substrate flux to fuel brown adipose tissue activity the IIA+ mice. These data illustrate that PLA2G2A elevates thermogenesis in an adipose tissuespecific manner, in part through elevated fuel utilization and increased mitochondrial content in BAT.
\end{abstract}

\section{Document Type}

Dissertation

Degree Name

Doctor of Philosophy (PhD)

Program

Biomedical Sciences

Research Advisor

Park, Edwards 


\section{Keywords}

Brown Adipose Tissue, Metabolism, Mitochondria, Obesity, Uncoupling

\section{Subject Categories}

Medicine and Health Sciences | Pharmacology 
University of TENNESSEE HeAlth SCIENCE CENTER

DOCTOR OF PHILOSOPHY DISSERTATION

\section{The Role of Secretory Phospholipase A2 Group IIA in Obesity and Metabolism.}

Author:

Michael S. Kuefner
Advisor:

Edwards A. Park

A Dissertation Presented for The Graduate Studies Council of The University of Tennessee Health Science Center in Partial Fulfillment of the Requirements for the Doctor of Philosophy degree from The University of Tennessee

$$
\text { in }
$$

Biomedical Sciences: Molecular and Systems Pharmacology College of Graduate Health Sciences 
Chapter 2 (C) 2017 by American Society for Biochemistry and Molecular Biology. Chapter 3 (C) 2019 by the Federation of American Societies for Experimental Biology. All other material (C) 2019 by Michael S. Kuefner. All rights reserved. 


\section{DEDICATION}

This dissertation is dedicated to my incredible parents, Mark and Christie. Thank you for everything you have done for Matt, Pat and me. Without your constant love and support none of this would be possible. 


\section{ACKNOWLEDGEMENTS}

I would like to acknowledge Dr. Edwards Park for his invaluable mentorship and guidance throughout my degree completion and for his assistance with this dissertation. I would also like to recognize collaborators Dr. Marshall B. Elam, Dr. Rajendra Raghow, and Dr. Erin Stephenson, as well as committee members Dr. Kafait U. Malik, Dr. Joan Han, Dr. Valeria Vasquez, and Dr. George Cook for their work and input on a variety of experiments included in my dissertation. Lastly, I would like to thank my former lab members Kevin Pham, Dr. Xiong Deng, and Dr. QingMing Dong for their contributions to the research reported here. 


\begin{abstract}
Secretory phospholipase $\mathrm{A}_{2}$ group IIA (PLA2G2A) is a member of a family of secretory phospholipases previously implicated in inflammation, atherogenesis, and antibacterial actions. These enzymes hydrolyze glycerophospholipids at the $s n-2$ position releasing lysophospholipids and fatty acids. Though studies have shown PLA2G2A is pro-inflammatory and promotes atherosclerosis, no research has analyzed the role of this enzyme in obesity and metabolism. Studies in the past 5-10 years utilizing various knockout or over-expression mouse models have analyzed the role of different secretory phospholipase $\mathrm{A}_{2} \mathrm{~S}\left(\mathrm{sPLA}_{2}\right)$ in metabolic diseases. From these studies, it is known that at least seven of the $11 \mathrm{sPLA}_{2}$ isozymes contribute to the prevention or causation of metabolic diseases such as obesity and Type 2 diabetes.

Increased energy expenditure, insulin sensitivity, and glucose tolerance are common characteristics of a mouse with a beneficial metabolic phenotype. We studied the impact of a high-fat diet on wild-type C57BL/6 and C57BL/6 mice over-expressing the human PLA2G2A gene (IIA+ mice). Despite no differences in food intake, the IIA+ mice weighed less, had increased oxygen consumption and energy expenditure, increased glucose tolerance and insulin sensitivity, and significantly less fat mass. There was a significant induction of uncoupling protein 1 (UCP1) in the brown adipose tissue (BAT) of IIA + mice, suggesting enhanced mitochondrial uncoupling in their adipose tissue to drive this metabolic phenotype. Previous work in Dr. Park's laboratory suggested that hepatic PLA2G2A expression is negatively regulated by acute thyroid hormone administration. We also examined the regulation of PLA2G2A and the metabolic changes that occur in response to variations in thyroid status. The impact of PLA2G2A on brown adipose tissue thermogenic gene expression was explored. C57BL/6 and IIA+ mice were made hypothyroid over a 10-week period or treated with thyroid hormone (T3) for 5 weeks. There were no significant changes in PLA2G2A abundance in response to thyroid status. The hypothyroid IIA + mice did not increase their energy expenditure even with T3 administration. However, the energy expenditure, substrate utilization, insulin sensitivity and glucose tolerance were all elevated in the IIA+ mice given T3. Moreover, white adipocytes from IIA+ mice were much more prone to 'beiging,' including increased expression of brown adipose thermogenic markers such as uncoupling protein 1 (UCP1), PR domain containing 16 (PRDM-16), and early B cell factor 2 (EBF2). Finally, the brown adipose tissue of IIA + mice had increased UCP1. Further metabolic analysis of $\mathrm{C} 57 \mathrm{BL} / 6$ and IIA+ mice revealed that even at thermoneutrality the IIA + mice display elevated oxygen consumption and energy expenditure. Moreover, citrate synthase activity was increased in the brown adipose tissue but not muscle in IIA+ animals, suggesting that the elevated thermogenesis is adipose tissue-specific. Finally, RNA microarray analysis of brown adipose tissue from C57BL/6 and IIA+ mice showed induction of numerous genes related to fuel substrate transport and metabolism, indicating an increase in substrate flux to fuel brown adipose tissue activity the IIA+ mice. These data illustrate that PLA2G2A elevates thermogenesis in an adipose tissue-specific manner, in part through elevated fuel utilization and increased mitochondrial content in BAT.
\end{abstract}


We discovered a novel role for PLA2G2A as an anti-obesogenic secretory phospholipase. My data indicate that PLA2G2A protects from diet-induced obesity and insulin resistance primarily through increased BAT activity and 'beiging' of WAT, resulting in improved whole-body thermogenesis. The rise in thermogenesis likely drives increased glucose utilization by various adipose depots, decreasing blood glucose which enhances glucose tolerance and insulin sensitivity. Further studies should be done to determine any specific fatty acids and/or lysophospholipids that may be released in response to PLA2G2As activity which may be driving this metabolic effect. 


\section{TABLE OF CONTENTS}

\section{CHAPTER 1. THE FUNCTIONS OF PLA2G2A AND SPLA2S IN

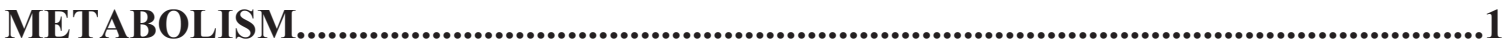

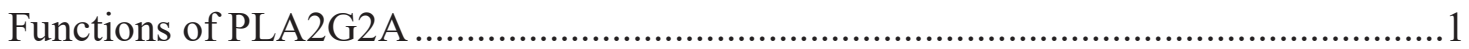

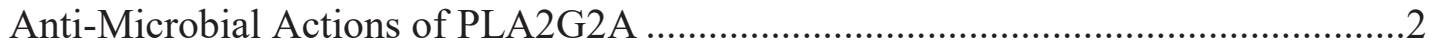

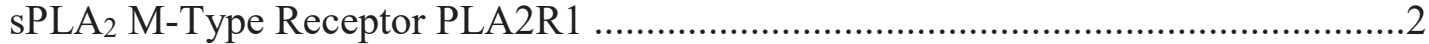

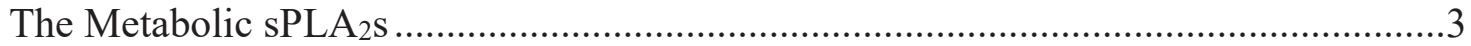

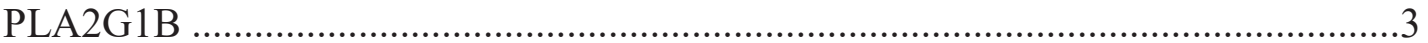

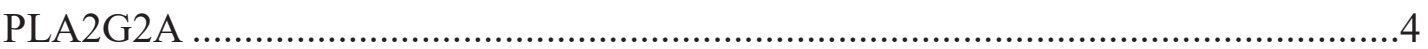

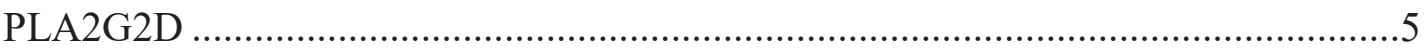

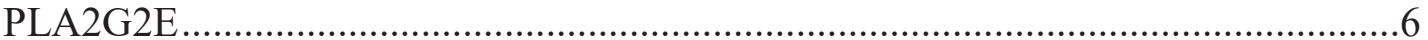

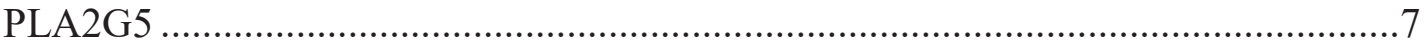

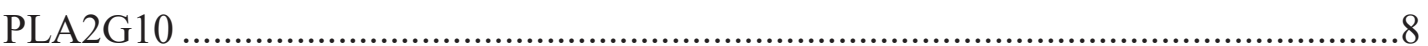

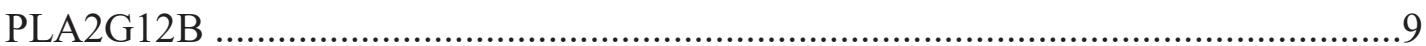

Concluding Remarks on Metabolic sPLA $2 \mathrm{~S}$......................................................... 10

\section{CHAPTER 2. PLA2G2A MODULATES INSULIN SENSITIVITY AND}

METABOLISM............................................................................................................12

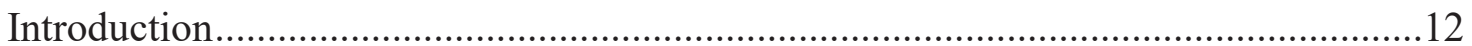

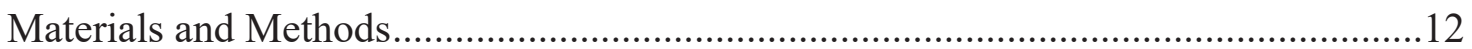

PLA2G2A Expressing C57BL/6 Mice (IIA+ mice) ……….....................................12

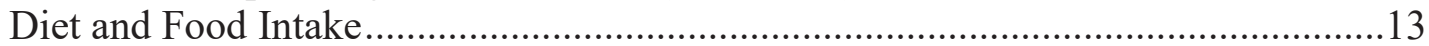

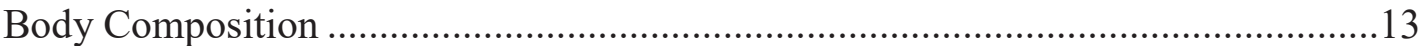

Glucose and Insulin Tolerance Tests ...................................................................13

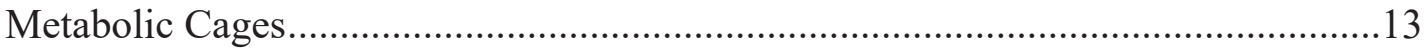

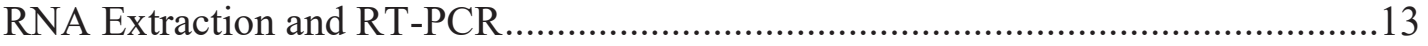

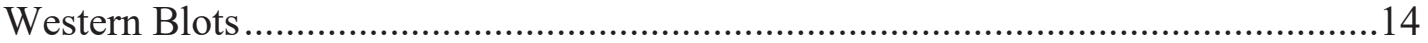

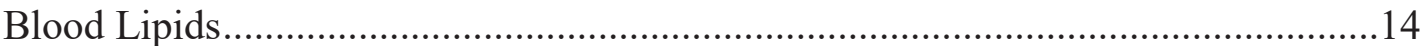

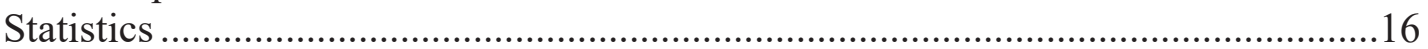

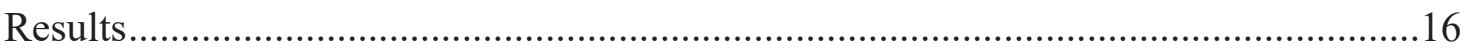

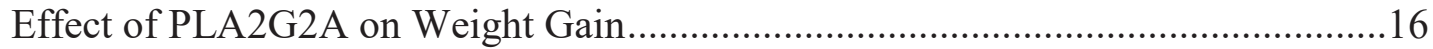

PLA2G2A Stimulates Energy Expenditure …………………………………........16

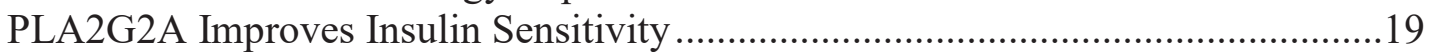

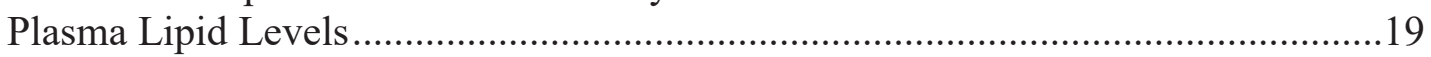

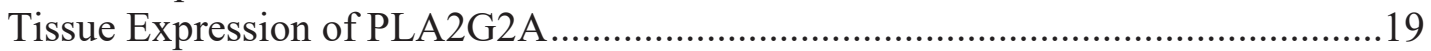

Metabolic Gene Expression in Liver of IIA+ Mice ………….................................23

Thermogenic Protein Expression in BAT ..............................................................23

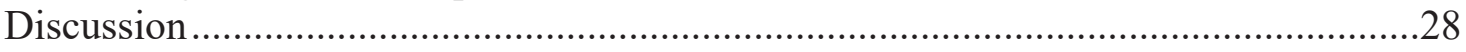

CHAPTER 3. PLA2G2A ENHANCES THE METABOLIC RATE AND INCREASES GLUCOSE UTILIZATION IN RESPONSE TO THYROID

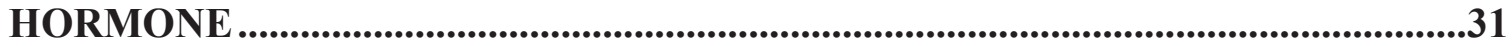

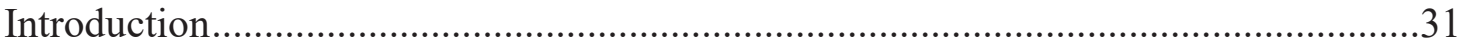

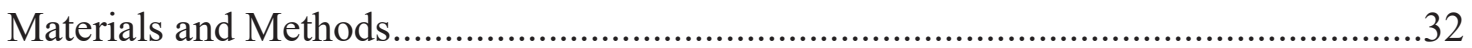




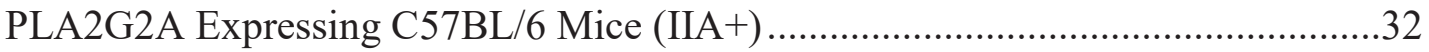

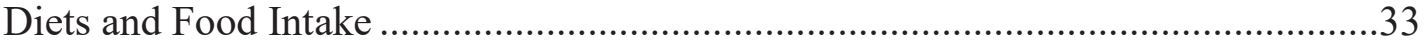

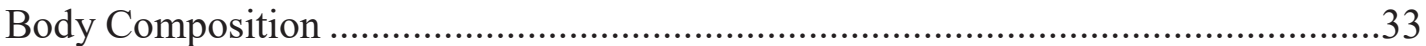

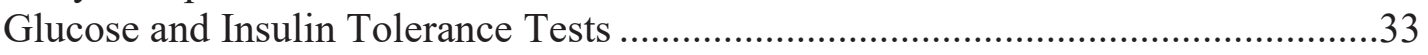

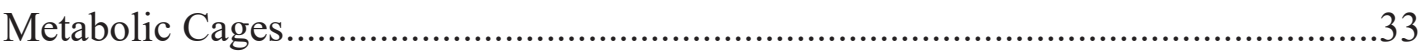

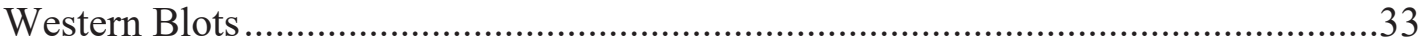

Thyroid Hormone Levels ....................................................................................

Primary Culture of Adipocytes ............................................................................34

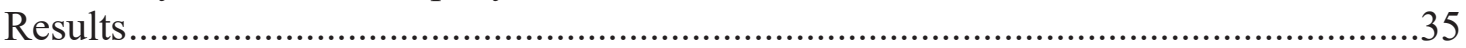

PLA2G2A Decreases Body Weight and Fat Mass …………………………….......35

PLA2G2A Increases Energy Expenditure and Carbohydrate Utilization with T3 ....35

Increased Insulin Sensitivity and Glucose Tolerance in IIA+ Mice ...........................39

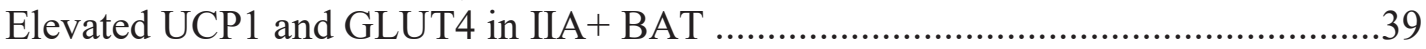

Thermogenic Protein Expression in IIA+ Adipocytes ..............................................42

Hepatic Protein Expression in IIA+ Mice ...........................................................42

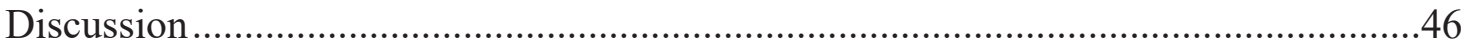

\section{CHAPTER 4. PLA2G2A AUGMENTS ADIPOSE TISSUE THERMOGENESIS ..50}

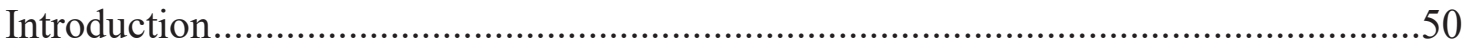

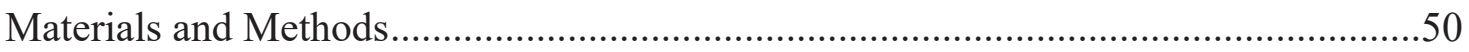

PLA2G2A Expressing C57BL/6 Mice (IIA+) ....................................................50

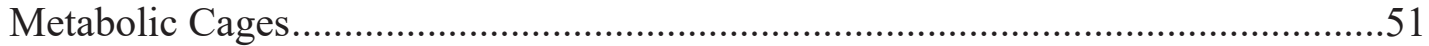

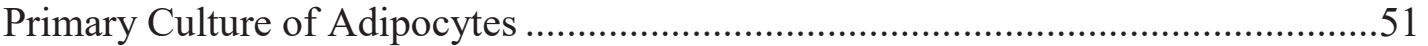

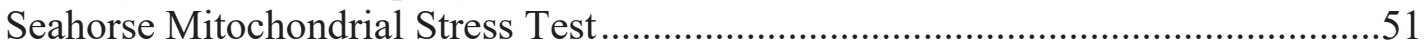

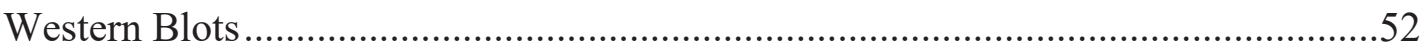

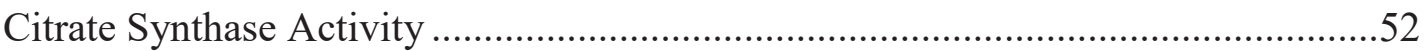

Recombinant PLA2G2A Enzyme ……………………….............................52

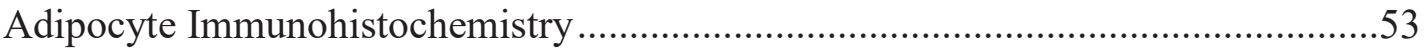

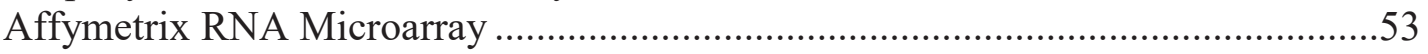

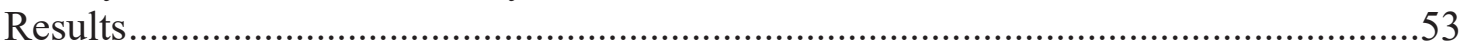

IIA+ Energy Expenditure at Thermoneutrality .....................................................53

Citrate Synthase Activity in BAT, WAT, and Muscle ………………………........55

Mitochondrial Complex Protein Expression in BAT..............................................55

Improved Mitochondrial Respiration in IIA+ Adipocytes..........................................55

IIA+ Adipocyte Protein Expression...........................................................................55

Recombinant PLA2G2A Enzyme Improves Thermogenic Protein Expression in

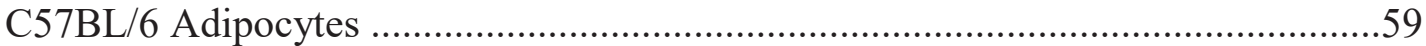

Changes in Substrate Transport and Chemokine Production in IIA+ BAT................59

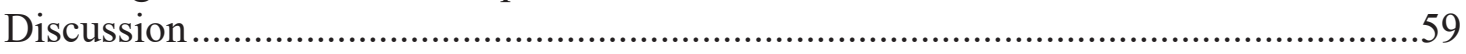

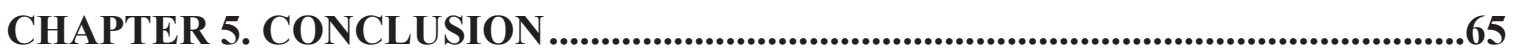

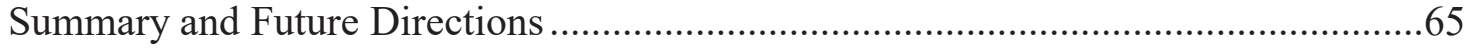

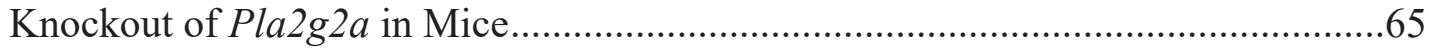

Impact of sPLA $\mathrm{P}_{2}$ Knockdown in Brown and White Adipocytes .................................66

Tissue-specific Effect of PLA2G2A Expression in the Liver and BAT......................66

Identification of Lysophospholipids and FFAs in IIA+ BAT .....................................66 
LIST OF REFERENCES ................................................................................................67

VITA 


\section{LIST OF TABLES}

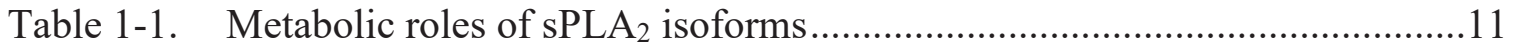

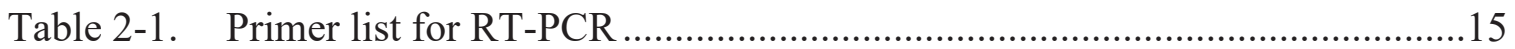




\section{LIST OF FIGURES}

Figure 2-1. Effects of PLA2G2A on body composition with HFD.................................17

Figure 2-2. Metabolic cage analysis of C57BL/6 and IIA+ mice on HFD .....................18

Figure 2-3. PLA2G2As effect on insulin and glucose tolerance .....................................20

Figure 2-4. Plasma lipid levels of C57BL/6 and IIA+ mice.........................................21

Figure 2-5. Tissue expression of PLA2G2A in IIA + mice...........................................22

Figure 2-6. Metabolic gene expression in IIA+ mice on HFD ......................................24

Figure 2-7. Metabolic protein expression in IIA+ mice on HFD ....................................25

Figure 2-8. Hepatic expression of insulin signaling markers .......................................26

Figure 2-9. Thermogenic protein expression induced in IIA+ BAT …….......................27

Figure 3-1. Serum T3 levels in mice on various thyroid diets.........................................36

Figure 3-2. Body composition of IIA+ mice in response to thyroid diets ........................37

Figure 3-3. Metabolic cage analysis of mice on various thyroid diets ............................38

Figure 3-4. ITTs and GTTs of mice on various thyroid diets.........................................40

Figure 3-5. Thermogenic and substrate transport protein expression in BAT ................41

Figure 3-6. Thermogenic protein expression elevated in IIA+ adipocytes .....................43

Figure 3-7. Differentiation timeline of C57BL/6 and IIA+ adipocytes............................4

Figure 3-8. Hepatic protein expression in mice on various thyroid diets .........................45

Figure 4-1. IIA+ mice have elevated energy expenditure at thermoneutrality .................54

Figure 4-2. Citrate synthase activity increased in IIA+ BAT ........................................56

Figure 4-3. Mitochondrial complex proteins induced in IIA+ mice.................................57

Figure 4-4. Mitochondrial respiration elevated in IIA+ white and brown adipocytes ....58

Figure 4-5. Thermogenic protein expression elevated in IIA + mice...............................60

Figure 4-6. Addition of PLA2G2A to brown adipocytes increases UCP1 expression....61

Figure 4-7. Changes in BAT transcriptome in the IIA+ mice 


\section{CHAPTER 1. THE FUNCTIONS OF PLA2G2A AND SPLA2S IN METABOLISM}

Phospholipase $\mathrm{A}_{2} \mathrm{~S}\left(\mathrm{PLA}_{2} \mathrm{~s}\right)$ are a group of enzymes that hydrolyze the $s n-2$ acyl bond in phospholipids to release non-esterified free fatty acids and lysophospholipids. There are four phospholipase $\mathrm{A}_{2}$ families including secretory phospholipase $\mathrm{A}_{2}\left(\mathrm{sPLA}_{2}\right)$, cytosolic phospholipase $\mathrm{A}_{2}$ (cPLA $)$, calcium-independent PLA 2 (iPLA 2 ), and platelet activating factor-acetylhydrolase (PAF-AH), also known as lipoprotein-associated phospholipase $\mathrm{A}_{2}$ (LpPLA 2 ). Secretory phospholipases $\mathrm{A}_{2}$ are low molecular weight enzymes that require millimolar amounts of $\mathrm{Ca}^{2+}$ for their activity. Currently, eleven ${ }_{\text {SPLA }}$ isoforms have been identified and of these ten are catalytically active (IB, IIA, IIC, IID, IIE, IIF, III, V, X, XIIA), while one is inactive (XIIB). Over one third of the $\mathrm{PLA}_{2}$ enzymes belong to the $\mathrm{SPLA}_{2}$ family. These secretory enzymes possess variable tissue-specific expression patterns and phospholipid substrate preferences, suggesting that each has distinct biological roles. Over the last 30 years, great progress has been made in defining the biological roles of sPLA $_{2}$ isoforms in a variety of pathologies. In addition, recent studies have discovered that some $\mathrm{sPLA}_{2}$ isoforms modulate obesity and metabolism through the production of lipid mediators that impact lipid mobilization, fatty acid oxidation, and other processes related to obesity and metabolic syndrome (MetS). Previous reviews on $\mathrm{sPLA}_{2} \mathrm{~s}$ have described their properties, subcellular localizations (13 ) and multitude of functions $(4,5)$.

Secretory phospholipase $\mathrm{A}_{2}$ group IIA (PLA2G2A) is a member of the $\mathrm{sPLA}_{2}$ family that participates in a variety of biological processes including atherogenesis, cardiovascular disease, anti-microbial actions, and may be involved in tumor suppression as well. The first portion of chapter 1 will review the function and past research on PLA2G2A focusing on its involvement in cardiovascular disease, inflammation, and actions as an anti-bacterial enzyme. The second section of this chapter will discuss recent studies highlighting the role that other $\mathrm{SPLA}_{2}$ isoforms have in the modulation of metabolic diseases such as obesity and metabolic syndrome.

\section{Functions of PLA2G2A}

sPLA2 group IIA (PLA2G2A) was first purified from the platelets and synovial fluids of patients suffering from arthritis (6). It has high affinity for anionic phospholipids such as phosphatidylserine (PS), phosphatidylethanolamine (PE), and phosphatidylglycerol (PG) (3), suggesting that membrane phospholipids, which are rich in phosphatidylcholine (PC) on the outer membrane (7), may not be a primary substrate for PLA2G2A. Many cell types, including hepatocytes, vascular smooth muscle cells, and endothelial cells, secrete PLA2G2A $(1,8)$. PLA2G2A can also act as a ligand, where it will bind with high affinity to the M-type receptor (PLA2R1), which is expressed in muscle, lung, spleen, and kidney $(9,10)$. Expression of PLA2G2A is induced by various stimuli including interleukin (IL)-1, IL-6, tumor necrosis factor (TNF $\alpha$ ), lipopolysaccharides, and cyclic AMP (9-12). The finding that PLA2G2A is highly 
abundant in biological fluids of patients suffering from inflammatory diseases, like arthritis and sepsis suggested that PLA2G2A promotes inflammation $(3,11,12)$. In support of this concept, under inflammatory conditions Pla2g2a knockout BALB/c mice have attenuated joint inflammation compared with wild-type BALB/c mice (13).

In addition to its inflammatory properties, PLA2G2A is proatherogenic with elevated levels of PLA2G2A being a biomarker for cardiovascular disease (14). Evidence of the participation of PLA2G2A in atherosclerosis came from work utilizing PLA2G2A transgenic mice. C57BL/6 (BL/6) mice do not express Pla2g2a due to frameshift mutation in exon 3. The human PLA2G2A gene was introduced into BL/6 mice to create PLA2G2A-expressing (IIA+) mice $(15,16)$. When fed a high cholesterol diet, the IIA+ mice had increased atherosclerotic lesions, reduced plasma high density lipoproteins (HDL) and slightly increased low density lipoproteins (LDL) (15). The hepatic cholesterol levels were elevated suggesting that PLA2G2A affects hepatic cholesterol uptake (17). Furthermore, bone marrow transplantation from IIA+ mice into LDL receptor-deficient mice increased atherosclerotic lesions, and expression of PLA2G2A in mouse macrophages accelerated the development of arterial wall lesions and the movement of cholesterol from LDL to foam cells $(18,19)$. Oral dosing of varespladib, an inhibitor of PLA2G2A, for 16 weeks reduced aortic atherosclerosis in ApoE-deficient mice (20). However, the failure of varespladib to reduce myocardial infarction and cardiovascular death in clinical trials has called into question the importance of PLA2G2A in atherogenesis $(21,22)$. Small nucleotide polymorphisms (SNPs) of secretory PLA2G2A have shown significant association with increased PLA2G2A expression, but not enzymatic activity, in patients with stable coronary heart disease, Type 2 diabetes, and recent cardiovascular surgical interventions $(23,24)$.

\section{Anti-Microbial Actions of PLA2G2A}

PLA2G2A was identified as an anti-microbial agent after its presence and bactericidal activity against gram-positive bacteria were detected in human tears (25). Subsequent studies have found that Pla2g2a was extremely effective at killing $L$. monocytogenes in Paneth cells of the intestine (26), as well as $S$. aureus in rabbit ascitic fluid (27). Importantly, the potent anti-microbial activity of PLA2G2A required $\mathrm{Ca}^{2+}$ and was only observed in gram-positive bacterial populations (25). The reason for this anti-

microbial activity is more than likely due to its substrate specificity. Phosphatidylglycerol is the principal component of gram-positive microbial membranes, and PLA2G2A hydrolyzes it with much higher efficacy compared to phosphatidylcholine which is typically abundant in mammalian cell membranes $(28,29)$.

\section{SPLA2 M-Type Receptor PLA2R1}

As mentioned previously, in addition to the enzymatic hydrolysis of glycerophospholipids, PLA2G2A can act as a ligand via binding to the sPLA2 receptor PLA2R1. This receptor may be a tumor suppressor gene critical to the induction of 
cellular senescence (30), a permanent form of cell-cycle arrest that prevents malignant cancer cell transformation and progression. Augert et al., found that with the knock-down of PLA2R1 using short hairpin RNA (shRNA), cells continued to grow in multiple tumor cell lines, but ectopic overexpression of PLA2R1 triggered premature cell senescence and prevented cell growth (30). The group also showed that PLA2G2A expression was induced 20-fold in senescent cells and overexpression induced cell senescence in a PLA2R1-dependent manner, suggesting that PLA2G2A may act as a ligand to suppress tumor growth (30). Interestingly, other studies have observed the involvement of PLA2G2A as an anti-cancer agent in gastric cancer $(31,32)$. Ganesan et al., detected a significant loss in PLA2G2A expression in late-stage cancers in vivo, and overexpression of PLA2G2A in the human gastric carcinoma cell line N87 dramatically inhibited tumor migration and invasion, suggesting that PLA2G2A may act as a general tumor suppressor in gastric cells (31). However, the authors made no mention of expression or involvement of the PLA2R1 receptor.

\section{The Metabolic sPLA2s}

\section{PLA2G1B}

Regarding secretory phospholipase A2 group IB, the role of PLA2G1B in obesity and metabolism has been elucidated by the studies from the Hui laboratory using Pla2g1b knockout (Pla2g1b-/-) mice or cell-specific PLA2G1B overexpression. PLA2G1B is primarily expressed in pancreatic acinar cells although it is also found in the lungs. The enzyme remains in an inactive state until it is released into the pancreatic juice in response to feeding. It is secreted into the intestinal lumen and proteolytically cleaved into its active form (33). Activated PLA2G1B contributes to lipid digestion and absorption within the digestive tract. When Pla $2 \mathrm{~g} 1 \mathrm{~b}$-/- mice on a C57BL/6 background were placed on a hypercaloric diet (58.5\% fat, $25 \%$ sucrose, $16.5 \%$ protein) for either 3 or 10 weeks, they were resistant to diet-induced obesity (34). The Pla2glb-/- mice showed a $37 \%$ reduction in plasma triglyceride (TG) levels primarily due to a decrease in hepatic VLDL production and an increase in TG-rich lipoprotein clearance. Pla $2 g 1 b$-/mice also displayed a $61 \%$ reduction in plasma cholesterol following 10 weeks on the hypercaloric diet compared to age-matched wild-type controls. It is noteworthy that Pla2g $1 b$-/- Ldlr-/- mice fed the same hypercaloric diet for 10 weeks displayed a similar phenotype, including reductions in fasting glucose, insulin, and plasma lipid levels (35). Moreover, wild-type mice consuming a high-fat, high-carbohydrate diet supplemented with the general sPLA2 inhibitor methyl indoxam showed a drastic reduction in body weight after 10 weeks (36). This decrease in body weight was accompanied by enhanced glucose tolerance and suppression of post-prandial plasma lysophospholipid levels. Importantly, transgenic mice over-expressing the human PLA2G1B in pancreatic acinar cells gained significantly more weight when given the hypercaloric high-fat/high-carb diet, and these mice also had reduced glucose tolerance and insulin resistance (37). 
The evidence supporting PLA2G1B inhibition as an avenue for improving metabolic health is quite strong. Mechanistically, these effects are mediated through the action of PLA2G1B on dietary phosphatidylcholine. Absorption of lysophosphatidylcholine (LPC) into the portal blood, plasma, and livers of Pla $2 \mathrm{~g} 1 \mathrm{~b}-/-$ mice was significantly reduced in mice that were fasted for 12 hours followed by a glucose-lipid mixed meal (38). These data suggest that phospholipid digestion in the intestinal lumen and absorption of the digested lysophospholipid product through the portal blood is enhanced by Pla2glb enzymatic activity (38). As pointed out by Richmond et al., while Pla2g1b is the major enzyme for phospholipid hydrolysis within the intestinal lumen, other lipolytic enzymes may partially compensate in its absence to preserve lipid and cholesterol absorption $(38,39)$. In regard to the enhanced glucose tolerance in Pla2g $1 \mathrm{~b}$-/- mice, there is evidence that LPC alone has a negative impact on hyperglycemia as shown with a glucose tolerance test (GTT) (38). Furthermore, the Pla $2 \mathrm{~g} 1 \mathrm{~b}$-/- mice have elevated fatty acid oxidation which can be directly suppressed by administration of LPC, suggesting that Pla2g1b enzymatic products reduce fatty acid oxidation (40).

Taken together, multiple studies on the metabolic impact of Pla2g1b inhibition indicate that it can dramatically reduce obesity in response to high fat feeding. Recent experiments have also demonstrated that the benefits of PLA2G1B inhibition mimic those seen in response to bariatric surgery in mice, including prevention of dyslipidemia as well as protection and remission from diet-induced Type 2 diabetes (41).

\section{PLA2G2A}

The roles of PLA2G2A in inflammation, obesity and metabolism continue to be defined (42). PLA2G2A is induced by several cytokines and second messengers including interleukins 1 and 6 (IL-1 and IL-6), tumor necrosis factor (TNF $\alpha$ ), lipopolysaccharides (LPS), and cyclic AMP suggesting a pro-inflammatory role (43-46). The Pla2g2a knockout (Pla2g2a-/-) BALB/c mice have significantly less joint inflammation than their wild-type counterparts with inflammatory stimuli (13). Elevated Pla2g2a is also associated with atherosclerosis as Pla2g2a participates in the conversion of LDL to the more atherogenic oxidized LDL (oxLDL) (47). In human studies, elevated PLA2G2A has been proposed to be a biomarker for cardiovascular disease since high levels of plasma PLA2G2A are a predictor for early stage atherosclerosis $(14,48)$. PLA2G2A polymorphisms have also been associated with hypercholesterolemia and subclinical atherosclerosis in patients with MetS (49).

With the focus of PLA2G2A studies being on its role in inflammation and atherosclerosis, studies on the actions of PLA2G2A in obesity and metabolism are quite limited. One consistent observation in the metabolic studies involving PLA2G2A is that its expression is significantly up-regulated in response to a high-fat diet $(46,50,51)$. In male Wistar rats, Pla2g2a expression was elevated 6-fold after 16 weeks on a highcarbohydrate high-fat (HCHF) diet (50). At the 8-week point, these rats were orally administered the PLA2G2A inhibitor KH064 which drastically reduced weight gain, fat 
mass, and prevented the increase in adipocyte crown formation and macrophage infiltration seen in the wild-type rats (50). The study also found that inhibition of Pla2g2a by KH064 was accompanied by an increase in lipolytic gene expression, attributed to an increase in hormone-sensitive lipase (HSL) phosphorylation. Treatment with KH064 also improved glucose tolerance and insulin sensitivity as shown by GTTs and ITTs (50).

Recently, we studied the metabolic phenotype of C57BL/6 mice that were genetically engineered to overexpress human PLA2G2A. In these mice, PLA2G2A improved the metabolic parameters and obesogenic symptoms $(51,52)$. C57BL/6 mice do not express the murine Pla2g2a due to a frameshift mutation in exon 3 (16). Overexpression of human PLA2G2A protected mice from weight gain on a chow or high-fat diet compared to wild-type C57BL/6 mice after 10 weeks. PLA2G2A expression also enhanced oxygen consumption (VO2) and energy expenditure. The expression of thermogenic genes in brown adipose tissue (BAT), including uncoupling protein 1 (UCP1), peroxisome proliferator-activated receptor $\gamma$ coactivator $1 \alpha(\mathrm{PGC}-1 \alpha)$, and Sirtuin-1 (SIRT1) was elevated $(51,52)$. PLA2G2A-expressing primary adipocytes from epididymal and inguinal white adipose tissue (WAT), and interscapular BAT showed elevated protein expression of several genes involved in adaptive thermogenesis compared to C57BL/6 wild-type adipocytes(52). The PLA2G2A-expressing mice showed significant improvements in glucose disposal and insulin tolerance based on GTTs and ITTs. To accompany this phenotype, mice expressing PLA2G2A also had reduced 6-hour fasting blood glucose levels and an increase in glucose transporter type 4 (GLUT4) in BAT suggesting that PLA2G2A enhances BAT glucose utilization (52).

There is abundant evidence that PLA2G2A contributes to the inflammatory response, but the enzyme's role in obesity and metabolism is still unclear. The current investigations of PLA2G2As metabolic role used different designs. The human PLA2G2A gene was expressed in mice, whereas the rat Pla2g2a enzyme was inhibited pharmacologically $(50,51)$. It is possible that overexpression of PLA2G2A in mice alters the expression of other secretory phospholipases in different tissues, which could influence metabolism. Similarly, the activity of various sPLA2 isozymes has not been examined in response to PLA2G2A inhibition by KH064. Moreover, food intake following KH064 administration was not reported in this study, and the impact of KH064 on intestinal lipid absorption was not assessed. Any of these factors may have contributed to the different metabolic outcomes of these studies of PLA2G2A.

\section{PLA2G2D}

Currently, no direct evidence on the function of PLA2G2D in obesity exists. However, it is known that this isoform, called the "resolving-sPLA2," plays a significant role in mediating inflammation in mice (53). PLA2G2D is abundantly expressed in lymphoid organs (53-56). Based on studies using Pla2g2d-/- mice, the resolution of hapten-induced contact hypersensitivity (CHS), a marker of the inflammatory response, was markedly impaired due to up-regulation of Th1 cytokines Ifng and IL12a in the lymph nodes of Pla2g2d deficient mice (53). The lipid profiles from lymph nodes of 
Pla2g2d-/- mice were altered, as arachidonic acid (AA), eicosapentaenoic acid (EPA), docosahexaenoic acid (DHA), and the DHA-derived metabolite resolvin D1 (RvD1) were significantly reduced in the Pla2g2d-/- mice. The addition of recombinant PLA2G2D protein to lipids extracted from lymph nodes resulted in a decrease in phosphatidylethanolamine (PE) species containing AA and DHA, suggesting that PLA2G2D preferentially hydrolyzes polyunsaturated fatty acid (PUFA)-containing PE (53).

Although there are no investigations on the participation of PLA2G2Ds in metabolism, its preferential enzymatic activity to release beneficial $\omega-3$ PUFAs and the lipid mediators is intriguing. A substantial body of literature indicates that $\omega-3$ PUFAs (primarily DHA, EPA) are favorable for metabolic health with respect to obesity and MetS $(57,58)$. Specifically, supplementation with $\omega-3$ PUFAs has demonstrated a protective role against high fat diet (HFD)-induced insulin resistance (59-62) and may elevate $\beta$-oxidation (63). In humans, circulating levels of $\omega$-3 PUFAs are elevated in healthy individuals when compared to patients with MetS, classifying low $\omega$-3 PUFA levels as a risk for the development of MetS (64). The presence of DHA metabolite RvD1 positively impacts metabolism as well $(65,66)$. RvD1 levels in adipose tissue are markedly diminished in obese mice, and addition of RvD1 to isolated epididymal fat pads from these animals ex vivo reduced expression of numerous pro-inflammatory signaling molecules while elevating the adipokine adiponectin, a signaling molecule that has numerous metabolic actions to combat obesity and Type 2 diabetes (65). Administration of RvD1 to $d b / d b$ mice resulted in improved glucose tolerance and insulin sensitivity (66).

Based on the lipid analysis described above, products produced by PLA2G2D enzymatic activity may be advantageous with respect to obesity and MetS as a whole, principally through their actions in adipose tissue. While PLA2G2D expression and activity is primarily restricted to lymph nodes, it is important to note that a significant portion of lymph are embedded and attached to mammalian adipose tissue $(67,68)$, indicating potential for immediate crosstalk between the two organs. Nonetheless, an analysis on the disruption of PLA2G2D activity needs to be carried out to fully understand its role in obesity and MetS.

\section{PLA2G2E}

The expression of PLA2G2E is elevated in the white adipose tissue (WAT) and BAT of female C57BL/6 mice fed a HFD (69). Studies using female Pla2g2e-/- mice found that they gained less weight on HFD over 18 weeks, with marked reductions in fat mass, hepatic lipid deposition, plasma aspartate aminotransferase (AST) and alanine aminotransferase (ALT) levels. PLA2G2E preferentially hydrolyzes PE and PS on very low density lipoproteins (VLDL), LDL, and HDL, although with weak enzymatic activity compared to that of other sPLA2s $(70,71)$. Mass spectrometry analysis of the lipoprotein particles from Pla2g2e-/- mice revealed a reduction in phospholipids (PL), triglyceride (TG), and cholesterol accumulation in VLDL, LDL, and HDL (69). These data suggest that Pla2g2e promotes obesity through elevated hepatic lipogenesis and VLDL assembly 
in the liver. It was previously reported that anionic phospholipids decrease the affinity for ApoE to bind the LDL receptor (LDL-r), which could impact lipoprotein particle clearance (72). Finally, whether lysophosphatidylserine (LPS) or lysophosphatidylethanolamine (LPE) impacts metabolism has yet to be determined.

In contrast to the work described above, another study using male Pla2g2e-/mice discovered that the enzyme plays a significant role in the regulation of lipolysis in adipocytes, likely through enhanced ERK1/2 signaling (73). Pla2g2e-/- mice had increased epididymal fat compared to C57BL/6 wild-type mice and accumulated significantly more triglyceride (TG) in the stromal vascular fraction (SVF) isolated from adipose tissue as well. The group also overexpressed Pla2g2e in OP9 stem cells or treated 3T3-L1 cells with Pla2g2e protein and observed reduced lipid accumulation and increased release of free glycerol, indicative of elevated lipolysis. Interestingly, these Pla2g2e-/- animals had reduced ERK1/2 signaling and HSL, the intracellular lipase responsible for hydrolyzing TG to FFAs. Finally, treatment of adipocytes with mouse Pla2g2e protein induced ERK1/2 signaling, demonstrating that Pla2g2e regulates adipocyte lipolysis through ERK/HSL signaling (73).

Currently, our understanding of PLA2G2E's role in obesity in metabolism is limited to the two studies described above which report contrasting phenotypes with Pla2g2e-/- mice. One notable difference is that Sato et al. used only female mice for their metabolic characterization while Zhi et al. used only male mice $(69,73)$. This emphasizes the possibility for sex-specific differences in Pla2g2e activity and its impact on obesity. However, the differences may also reflect the differences in generation of the knockout mice, diets and animal facilities.

\section{PLA2G5}

PLA2G5, which is expressed in WAT and elsewhere, provides protection from diet-induced obesity. Sato et al. (69) found that PLA2G5 expression was elevated in response to feeding a HFD in female C57BL/6 mice. When placed on a HFD, the Pla2g5-/- mice gained a large amount weight gain that was primarily located in the visceral fat (69). In GTT and ITT, the Pla2g5-/- mice were less glucose tolerant and had increased insulin resistance. Furthermore, there was a striking induction of plasma ALT levels and hepatic fat deposition, indicating exacerbated hepatosteatosis (69). It has previously been shown that PLA2G5 preferentially hydrolyzes phosphatidylcholine (PC) in low-density lipoprotein (LDL) (74). In the Pla2g5-/- mice, phospholipid, cholesterol, and TG levels were considerably higher in LDL (69).

PLA2G5 modulates bone marrow derived macrophage (BMDM) polarization as well. BMDM were treated with palmitic acid or lipopolysaccharides to induce the inflammatory response. Addition of recombinant PLA2G5 enzyme augmented expression of M2 makers Arg1 and Cd206 in the BMDM, suggesting that PLA2G5 has antiinflammatory effects (69). The capacity for PLA2G5 to push macrophage polarization from an M1 to M2 state broadens its impact as the metabolic benefits of M2 macrophages 
are well documented (75-77). Genetic deletion of Th2 or M2 inducers increases the risk for metabolic disorders (76), and M2 macrophage infusion into obese mice has proven to be effective in treating obesity and improving insulin sensitivity (77). In humans, M2 macrophages are more prevalent in lean adipose tissue as well (75). While additional work needs to be done to elucidate the effects of PLA2G5 on metabolism, it appears that these actions are mediated in part by the fatty acid and/or PC-released induction of M2 macrophage polarization in adipose tissue.

The role of PLA2G5 in glucose-stimulated insulin secretion (GSIS) is complex and depends on the experimental model utilized. A recent study published by Shridas et al., discovered that GSIS was decreased in isolated pancreatic islets from Pla2g5 knockout mice and in pancreatic MIN6 cells following siRNA-mediated PLA2G5 knockdown (78). Additionally, PLA2G5 over-expression in the MIN6 cell line resulted in elevated GSIS and increased AA release into the media, despite no change in prostaglandin E2 (PGE2) compared to control cells (78). In contrast to the studies with isolated islets, the GSIS of Pla2g5-/- mice on a standard chow diet was significantly elevated compared to WT mice, which was attributed to increased pancreatic islet size and number of proliferating cells in the $\beta$-islets of the pancreas. The in vivo data from this study suggests a reduction in the release of AA, a fatty acid that is contained in over $30 \%$ of the glycerolipids in rodent islets (79) by Pla2g5 may be beneficial for insulin secretion and $\beta$-cell proliferation. Previous work has shown that AA generally induces GSIS, while inhibition of the release of AA inhibits GSIS (80). Interestingly, one of the major metabolites of AA is PGE2, a well-known inhibitor of GSIS (81). PGE2 interacts with the PGE2 receptor EP3 resulting in a decrease in adenylyl cyclase activity and subsequent reduction in cAMP $(82,83)$. These data show that PLA2G5 plays a role in insulin secretion and $\beta$-cell proliferation, which may be dependent on the amount of AA released versus the amount of AA used for PGE2 production.

\section{PLA2G10}

Studies with transgenic mice discovered that PLA2G10 mediates adipogenesis and strongly reduces corticosteroid production in mice, leading to the hypothesis that it may protect from diet-induced obesity and improve metabolism $(84,85)$. PLA2G10 binds to zwitterionic phospholipids such as $\mathrm{PC}$ with high affinity resulting in the potent release of AA and LPC. PLA2G10 is expressed in a variety of tissues including the lungs, adrenal glands, brain, heart, and adipose tissue (70, 86-89). Pla2g10-/- mice on a C57BL/6 background gain significantly more weight and fat mass over 40 weeks compared to the wild type mice even on a chow diet (84). Pre-adipocytes prepared from the WAT of Pla2g10-/- mice accumulated more TG when induced to differentiate ex vivo, suggesting that PLA2G10 has a direct effect in adipose tissue to reduce lipid accumulation. Similarly, when the OP9 cell line was modified to overexpress PLA2G10, the cells had considerably less TG accumulation following differentiation (84).

Interestingly, the reduction in TG accumulation may be attributed to PLA2G10s ability to generate lipid products that suppress liver X receptor (LXR)-induced adipogenic genes 
including sterol regulatory element-binding protein 1c (SREBP-1c), stearoyl-CoA desaturase-1 (SCD-1), and fatty acid synthase (FAS) (85, 90-92).

As mentioned previously, PLA2G10 is expressed in adrenal cells and has a regulatory role in adrenal corticosteroid production. Overexpression of PLA2G10 in C57BL $/ 6$ mice resulted in a $30-40 \%$ reduction in corticosteroid production, and this effect was reversed by methyl indoxam administration (85). Furthermore, PLA2G10 overexpression dramatically reduced expression of the LXR-target gene steroidogenic acute regulatory protein (StAR), a nuclear encoded mitochondrial protein that mediates the rate-limiting step of steroid synthesis (93).

The current data on PLA2G10 indicate it may alleviate symptoms of obesity and Type 2 diabetes in a bimodal fashion. First, PLA2G10 expression clearly reduces weight gain and overall adiposity in mice. The secondary effects come from its ability to decrease corticosteroid production. Excessive use or production of glucocorticoids will induce insulin resistance, weight gain and adiposity while also exacerbating Type 2 diabetes (93-96). Based on our current understanding of PLA2G10, it is a promising sPLA2 to favorably improve metabolism.

Pla2g10 is also expressed in the pancreatic beta cells and suppresses glucose stimulated insulin secretion (GSIS) (97). Like Pla2g5 which was discussed in the previous section, Pla2g10 generates AA. However, this pool of AA is converted to prostaglandin E2 which binds to the EP3 receptor. EP3 elevates cAMP leading to decreased insulin secretion. The exact reason that Pla2g5 and Pla2g10 have opposite effects on GSIS is unclear. However, Pla2g10 does have to be proteolytically activated and this may give it access to a different pool of phospholipids. Finally, older Pla2g10 $\mathrm{KO}$ mice appear to be protected from age related glucose intolerance.

The current data on PLA2G10 indicate it impacts multiple aspects of mouse metabolism and hormonal action. Regarding metabolism, PLA2G10 expression reduces weight gain and overall adiposity in mice. With respect to hormone actions, Pla2g10 decreases corticosteroid production. Excessive use or production of glucocorticoids will induce insulin resistance, weight gain and adiposity while also exacerbating Type 2 diabetes (93-96). However, Pla2g10 reduces GSIS giving this phospholipase a complex contribution to the overall metabolic state.

\section{PLA2G12B}

PLA2G12B is the only phospholipase implicated in metabolism showing no catalytic activity due to a point mutation in its active site, and thus it is hypothesized to act as a ligand for receptors that are currently unidentified $(98,99)$. PLA2G12B is highly expressed in the liver, small intestine and kidneys, and this tissue specific pattern of expression is consistent between humans and mice (98). Using Pla2g $12 \mathrm{~b}$-/- mice fed an ad libitum chow diet, Guan et al. discovered that knockout of the Pla2g12b gene increased TG, cholesterol, and free fatty acids in the liver, resulting in severe 
hepatosteatosis (100). Hepatocyte nuclear factor-4alpha (HNF-4 $\alpha$ ) and its co-activator PGC-1 $\alpha$ induce Pla2g $12 b$ expression $(100,101)$, resulting in the induction of genes involved in lipoprotein packaging (microsomal triglyceride transfer protein, MTP) and VLDL secretion $(102,103)$. Moreover, liver-specific $H N F-4 \alpha-/-$ mice are phenotypically very similar to the Pla2g $12 \mathrm{~b}-/$ - animals, as both lines have reduced serum TG and cholesterol levels and display severe hepatosteatosis $(100,104)$. These observations suggest that PLA2G12B is one gene involved in the control of lipid metabolism downstream of HNF-4 $\alpha$. Infection of mice with an adenovirus encoding Pla2g12b into Pla2g12b-/- mice improved hepatic VLDL secretion and restored the decline in serum TGs (100). These data indicate that Pla2g12b is under the regulation of HNF-4 $\alpha$ and plays an important role in metabolism by regulating lipoprotein packaging and VLDL secretion in the liver. However, it was recently discovered that PLA2G12B is negatively regulated by farnesoid X receptor (FXR), which controls bile acid and TG homeostasis in the liver (105). Drug-induced activation of FXR resulted in substantial repression of hepatic Pla2g12b in C57BL/6 mice. Pla2g12b repression was accompanied by a decrease in serum TGs, attributed to diminished de novo lipogenesis and hepatic secretion of TGrich VLDL (105). This suggests that down-regulation of Pla2g12b is part of the process by which hepatic FXR activation reduces VLDL-TG secretion. Importantly, HNF-4 $\alpha$ and FXR are functionally active and involved in metabolic processes in the small intestine, a tissue which shows strong expression of PLA2G12B, indicating that the phospholipase may have an impact in lipid metabolism, secretion and/or absorption there as well (106108).

\section{Concluding Remarks on Metabolic sPLA2s}

Recent advancements in the field of sPLA2s have made it evident that they have considerable impact on protection from or progression towards metabolic diseases such as obesity and metabolic syndrome, as shown in Table 1-1. One issue in analyzing the metabolic impact of sPLA2s is that many of the sPLA2s influence intertwined pathologies such as atherosclerosis, heart disease, and cancer $(3,75)$. For this reason, future studies on the SPLA2s should carefully consider the role they might play based on tissue localization, as their distinct functions may be altered based on the tissue being analyzed. Furthermore, the expression and compensation of additional sPLA2 isoforms in transgenic animal models is another factor that might result in large phenotypic changes, and thus should also be observed to advance what we know about sPLA2s in obesity, metabolism, and more. 
Table 1-1. Metabolic roles of SPLA 2 isoforms

\begin{tabular}{|c|c|c|c|}
\hline Isoforms & Primary localization & Metabolic implications & Literature \\
\hline PLA2G1B & Pancreas, lung & $\begin{array}{l}\text { Promotes weight gain; increases TG } \\
\text { and cholesterol levels through } \\
\text { elevated intestinal LPC absorption }\end{array}$ & $(34-41)$ \\
\hline PLA2G2A & $\begin{array}{l}\text { Platelets, liver, } \\
\text { leukocytes, paneth cells, } \\
\text { adipose tissue }\end{array}$ & $\begin{array}{l}\text { Controversial; promotes weight gain, } \\
\text { insulin resistance in rats. Improves } \\
\text { metabolic parameters in mice }\end{array}$ & $\begin{array}{l}(46,49- \\
52)\end{array}$ \\
\hline PLA2G2D & $\begin{array}{l}\text { Lymph tissue dendritic } \\
\text { cells }\end{array}$ & $\begin{array}{l}\text { Undocumented; May be } \\
\text { metabolically beneficial due to } \\
\text { release of anti-inflammatory } \\
\text { FAs/lipid mediators }\end{array}$ & $(53-56)$ \\
\hline PLA2G2E & Adipose, skin & $\begin{array}{l}\text { Controversial; Pla2g2 } e^{-/} \text {male mice } \\
\text { display blunted lipolysis and elevated } \\
\text { TG storage. Other experiments using } \\
\text { Pla2g2e } 2 e^{-/} \text {female mice found reduced } \\
\text { lipid accumulation }\end{array}$ & $\begin{array}{l}(69-71 \\
73)\end{array}$ \\
\hline PLA2G5 & $\begin{array}{l}\text { Adipose, bronchial } \\
\text { epithelial cells, } \\
\text { hepatocytes, islets, } \\
\text { macrophages, } \\
\text { cardiomyocytes }\end{array}$ & $\begin{array}{l}\text { Protects from diet-induced obesity } \\
\text { and insulin resistance; pushes adipose } \\
\text { tissue macrophages from } \mathrm{M} 1 \rightarrow \mathrm{M} 2 \\
\text { state }\end{array}$ & $(69,78)$ \\
\hline PLA2G10 & $\begin{array}{l}\text { Lung, adrenal gland, } \\
\text { brain, heart, adipose }\end{array}$ & $\begin{array}{l}\text { Protects from diet-induced obesity. } \\
\text { Elevates TG clearance in adipose and } \\
\text { suppresses glucocorticoid production } \\
\text { in adrenal cells }\end{array}$ & $\begin{array}{l}(70,84-86, \\
88,89)\end{array}$ \\
\hline PLA2G12B & $\begin{array}{l}\text { Liver, small intestine, } \\
\text { kidneys }\end{array}$ & $\begin{array}{l}\text { Strongly regulates hepatic lipoprotein } \\
\text { packaging and VLDL secretion; } \\
\text { expression protects from } \\
\text { hepatosteatosis }\end{array}$ & $\begin{array}{l}(98,100 \\
101,105)\end{array}$ \\
\hline
\end{tabular}




\section{CHAPTER 2. PLA2G2A MODULATES INSULIN SENSITIVITY AND METABOLISM*}

\section{Introduction}

Phospholipases A2 (PLA2s) are a group of esterase enzymes that hydrolyze the second carbon of membrane phospholipids to release nonesterified free fatty acids and lysophospholipids $(4,109)$. PLA2 enzymes have been classified into four groups based on their $\mathrm{Ca}^{2+}$ requirement and cellular localization. These include secretory phospholipase A2 (sPLA2), cytosolic PLA2, calcium-independent PLA2, and lipoprotein-associated PLA2 $(110,111)$. The sPLA2s are low molecular mass phospholipases $(14-18 \mathrm{kDa})$ that are secreted into the extracellular environment in response to various stimuli. Currently, eleven sPLA2s have been identified $(3,110)$. These phospholipases mediate multiple biologic actions by targeting various noncellular phospholipids, such as microbial membranes, dietary phospholipids, and lipoproteins (3).

As discussed in the introduction, there is a relationship between PLA2G2A, inflammatory processes, and cardiovascular disease. However, the contributions of PLA2G2A to metabolic regulation have not been examined. In this chapter, we describe my investigations into the metabolic effects of PLA2G2A in relation to hypercaloric high fat feeding. My findings demonstrated that IIA + mice are resistant to body weight and fat mass gains in response to a high fat diet. Moreover, IIA+ mice have superior glucose tolerance and insulin sensitivity, and these changes are associated with elevations in total energy expenditure. This study has identified a novel role of PLA2G2A in the regulation of whole-body metabolism.

\section{Materials and Methods}

\section{PLA2G2A Expressing C57BL/6 Mice (IIA+ mice)}

The IIA+ mice, which express the human PLA2G2A gene under the regulation of the human promoter, were provided by Dr. Eric Boilard at the Université Laval (CHUL), Quebec, Canada (46). Male mice heterozygous for the PLA2G2A gene were bred with $\mathrm{BL} / 6$ females. For these experiments, male BL/6 and IIA+ mice were used. Mice were placed on diets 8 weeks after birth. In each dietary group, we had $11 \mathrm{BL} / 6$ chow-fed mice, 10 IIA + chow-fed mice, $11 \mathrm{BL} / 6$ high fat diet-fed mice, and 12 IIA+ high fat dietfed mice. Mice were housed with a constant light and dark phase of $12 \mathrm{~h}$ at $20-23^{\circ} \mathrm{C}$.

*Reprinted from final submission with permission. Kuefner M.S., Pham K., Redd J.R., Stephenson E.J., Harvey I., Deng X., Bridges D., Boilard E., Elam M.B., Park E.A. Secretory phospholipase A2 group IIA modulates insulin sensitivity and metabolism. JLR, 2017; 58:1822-1833 https://doi.org/10.1194/jlr.M076141 (51). 


\section{Diet and Food Intake}

The chow diet was the Teklad LM-485 mouse/rat diet (7012) and the high fat diet was D12451 from Research Diets Inc. The high fat diet provided 45\% of the calories from fat, while the chow diet contained $5 \%$ of the calories as fat. The mice were given free access to food throughout the study. The food was weighed at weekly intervals and the kilocalorie consumption was calculated based on grams of food eaten, the calorie density of the food, and the weight of the mice.

\section{Body Composition}

Mice were weighed and total fat and fat-free mass were determined weekly by EchoMRI-1100.

\section{Glucose and Insulin Tolerance Tests}

Following $6 \mathrm{~h}$ fasts, baseline blood glucose concentrations were determined in blood collected from a small tail incision using a hand-held glucometer (AccuCheck). Dglucose in PBS [2 mg/kg fat-free mass; glucose tolerance test (GTT)] or insulin [1 U/kg fat-free mass; insulin tolerance test (ITT)] was injected intraperitoneally and blood glucose concentrations measured at 15, 30, 45, 60, 75, 90, 105, and 120 min postinjection (112). The data are presented as blood glucose levels versus time and the area under the glucose curve.

\section{Metabolic Cages}

After 11 weeks of diet, mice were individually housed in the comprehensive laboratory animal monitoring system (CLAMS) chambers. They were maintained on either chow or high fat diets. Total energy expenditure was determined using indirect calorimetry (113). VO2 and heat production are expressed relative to fat-free mass. The respiratory exchange ratio (RER) was measured to determine energy substrate preference. Physical activity was determined by the number of infrared beam breaks.

\section{RNA Extraction and RT-PCR}

Mice were allowed access to food prior to tissue collection for RNA and protein. The mice were anesthetized by isoflurane and then euthanized by cervical dislocation. Liver, quadriceps muscle, brown adipose tissue (BAT), and epididymal white adipose tissue (eWAT) were harvested and stored in liquid nitrogen. Total RNA was extracted from mouse liver by RNA-STAT 60 (Tel-Test). cDNA was synthesized via reverse transcription using Superscript III (Invitrogen). The parameters for RT-PCR were as follows: $95^{\circ} \mathrm{C}$ for $5 \mathrm{~min}, 40$ cycles of $95^{\circ} \mathrm{C}$ for $15 \mathrm{~s}, 60^{\circ} \mathrm{C}$ for $30 \mathrm{~s}$, and $72^{\circ} \mathrm{C}$ for $10 \mathrm{~s}$. 
Cyclophilin D was used as a reference gene. The quantification of the PCR products was carried out using the $\Delta \Delta \mathrm{Ct}$ method (114). The forward and reverse primers used for realtime PCR are shown in Table 2-1. The PLA2G2A primers were from Qiagen (catalog number PPH05823B).

\section{Western Blots}

Total protein from mouse livers, quadriceps, BAT, and eWAT was isolated in RIPA buffer containing protease inhibitors [50 mM Tris- $\mathrm{HCl}(\mathrm{pH}$ 7.4), $100 \mathrm{mM} \mathrm{NaCl}, 5$ mM EDTA (pH 8.0), 1\% Triton, $1 \mathrm{mM}$ benzamidine, and $0.5 \mathrm{mM}$ PMSF] (115). Protein lysates were prepared in loading buffer and equal amounts were loaded on 4-20\% gradient Tris/glycine precast acrylamide gels. Proteins were separated using SDS-PAGE and transferred to nitrocellulose membranes. Membranes were blocked with 5\% nonfat milk in TBS-T prior to antibody incubation. Membranes were immunoblotted with the appropriate primary antibody in 5\% BSA in TBS-T and secondary antibody in 5\% nonfat milk in TBS-T. Immunoreactive proteins were detected using Super Signal West Femto chemiluminescent substrate (Thermo Scientific) (114). The following antibodies were used: acetyl-CoA carboxylase (ACC1), catalog number C83B10 from Cell Signaling Technology; p-p70S6K(Thr389), catalog number 108D2 from Cell Signaling Technology; p70S6K, catalog number 9202 from Cell Signaling Technology; Akt, catalog number C67E7 from Cell Signaling Technology; $\beta$-actin, catalog number 13E5 from Cell Signaling Technology; uncoupling protein 1 (UCP1), catalog number D9D6X from Cell Signaling Technology; UCP2, catalog number D105V from Cell Signaling Technology; GAPDH, catalog number 14C10 from Cell Signaling Technology; alphatubulin, catalog number 11H10 from Cell Signaling Technology; p-Akt (T308), catalog number 244F9 from Cell Signaling Technology; PLA2G2A, catalog number AB23705 from AbCam; sirtuin 1 (SIRT1), catalog number A21993 from Life Technologies; SREBP-1c, catalog number 5351581 from BD Pharmingen.

\section{Blood Lipids}

Plasma cholesterol and lipoproteins in serum were measured at the University of Tennessee Endocrinology/Lipoprotein Laboratory. Blood was obtained by cardiac puncture from anesthetized mice and the serum was collected from the clotted blood. For hepatic triglyceride measurements, liver samples were first lysed in homogenization buffer [50 mM Tris (pH 8), $5 \mathrm{mM}$ EDTA, $30 \mathrm{mM}$ EDTA, $30 \mathrm{mM}$ mannitol, and phosphatase inhibitors]. Lipids were then extracted with a chloroform/methanol mixture using the Folch extraction procedure (116). Once the chloroform phase was evaporated, hepatic triglycerides were measured using the Sigma triglyceride assay kit as directed by the manufacturer. 
Table 2-1. Primer list for RT-PCR

\begin{tabular}{lll}
\hline Gene & Forward & Reverse \\
\hline Cyclophilin D & 5'-TGGAGAGCACCAAGACAGACA-3' & 5'-TGCCGGAGTCGACAATGAT-3' \\
Gapdh & 5'-AAGGTCATCCCAGAGCTGAA-3' & 5'-CTGCTTCACCACCTTCTTGA-3' \\
Hprt1 & 5'-CACAGGACTAGAACACCTGC-3' & 5'-GCTGGTGAAAAGGACCTCT-3' \\
Sirt1 & 5'-GCTCTAGTGACTGGACTCCG-3' & 5'-GCCACAGCGTCATATCATCC-3' \\
Ppargcla & 5'-GTCCTTCCTCCATGCCTGAC-3' & 5'-AGTGCTAAGACCGCTGCATT-3' \\
Srebf1 & 5'-GGAGCCATGGATTGCACATT-3' & 5'-GCTTCCAGAGAGGAGGCCAG-3' \\
Acaca & 5'-GGAGGAGGAGGGAAAGGGAT-3' & 5'-CTCCCCAAGGAGATACCCCA-3' \\
Cpt1a & 5'-GACGAATCGGAACAGGGATATAG-3' & 5'-GGGCTAGAGAACTTGGAAGAAA-3' \\
Ucp1 & 5'-GGCCCTTGTAAACAACAAAATAC-3' & 5'-GGCAACAAGAGCTGACAGTAAAT-3' \\
\hline
\end{tabular}




\section{Statistics}

Data analysis was performed using the JMP Statistical Discovery program. Data presented are the mean results of 3-4 replicate experiments \pm the SE. Data were analyzed by Student's one/two-tailed t-test or ANCOVA. P $<0.05$ was considered to be statistically significant.

\section{Results}

\section{Effect of PLA2G2A on Weight Gain}

Initially, we evaluated the effect of PLA2G2A on weight gain and lipid accumulation by monitoring the body composition in response to 10 weeks of high fat diet consumption in both BL/6 control and the IIA+ mice (13). In response to the high fat diet, BL/6 mice gained significantly more weight than the chow-fed BL/6 mice or the IIA + mice on either the chow or high fat diet. The IIA + mice were resistant to high fat diet-induced weight gain (Figure 2-1A). The fat-free mass of BL/6 and IIA+ mice was similar on both the chow and high fat diets (Figure 2-1B). BL/6 mice receiving the high fat diet had significantly elevated total body fat compared with their chow counterparts, whereas fat mass was slightly lower in the IIA+ groups regardless of the diet (Figure 2-1C). We measured the caloric intake of the mice and found that over the 10week feeding period the BL/6 and IIA+ mice consumed similar kilocalories of food per gram of body weight (Figure 2-1D). The unexpected observation was that the IIA+ mice on the high fat diet were protected from lipid accumulation, unlike the BL/6 mice on the high fat diet.

\section{PLA2G2A Stimulates Energy Expenditure}

Next, we assessed energy expenditure and physical activity using CLAMS. The BL/6 mice on the high fat diet had higher oxygen consumption than the chow-fed BL/6 mice (Figure 2-2A). Surprisingly, the IIA + mice on chow and high fat diets had higher oxygen consumption than the BL/6 controls, suggesting increased energy expenditure regardless of diet. In addition to the elevated VO2 consumption, the IIA+ mice had increased heat production (Figure 2-2B). The BL/6 and IIA + mice on the high fat diet had lower RER compared with their chow counterparts, indicating less carbohydrate utilization (Figure 2-2C). The activity levels of the IIA+ mice were similar to the BL/6 mice on the same diets, indicating the increased energy expenditure was not due to increases in activity (Figure 2-2D). However, the mice on the high fat diet were less active than those on the chow diet. Overall, the data in Figures 2-1 and 2-2 suggest that the lean phenotype of the IIA+ mice was due to elevated basal metabolism. 
A.
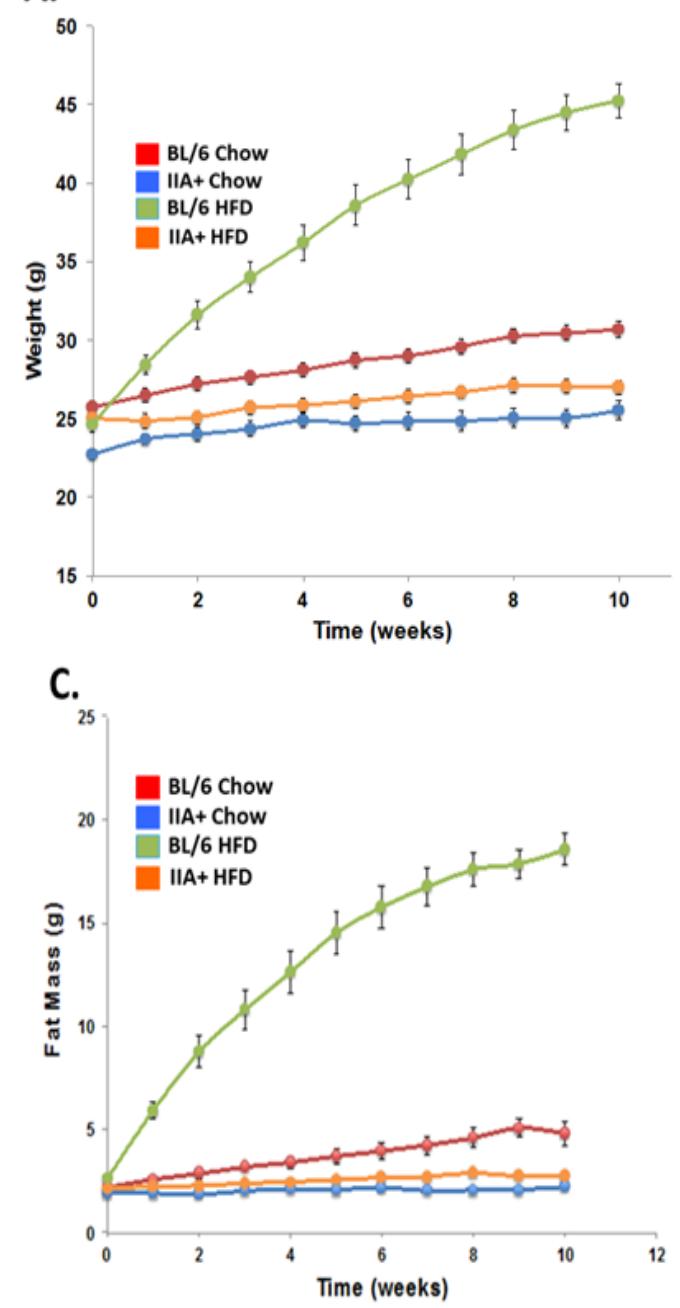

B.
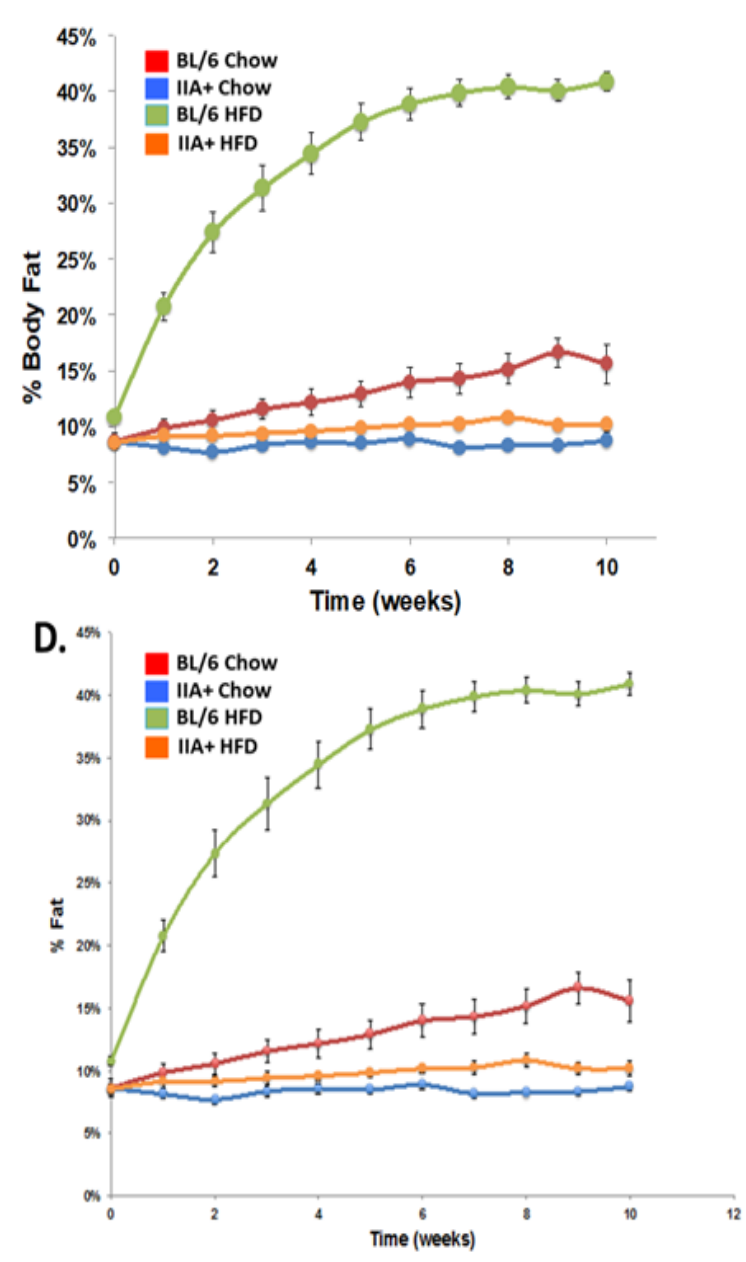

Figure 2-1. Effects of PLA2G2A on body composition with HFD

Weight gain and body composition of IIA + mice on high fat diets. BL/6 and IIA + mice were placed on chow diet or HFD for 10 weeks, as described in the Materials and Methods. A: The weight gain (grams) of the mice in the four groups was assessed on a weekly basis. B, C: MRI analysis was performed weekly and used to determine the lean mass and percent body fat. D: The average kilocalories consumed per mouse per gram of body weight were calculated from the food intake and the calorie content of the various diets. In all groups, there were 10-12 mice. The differences in the various time points were determined by two-way ANOVA, using genotype and diet as two separate factors. 


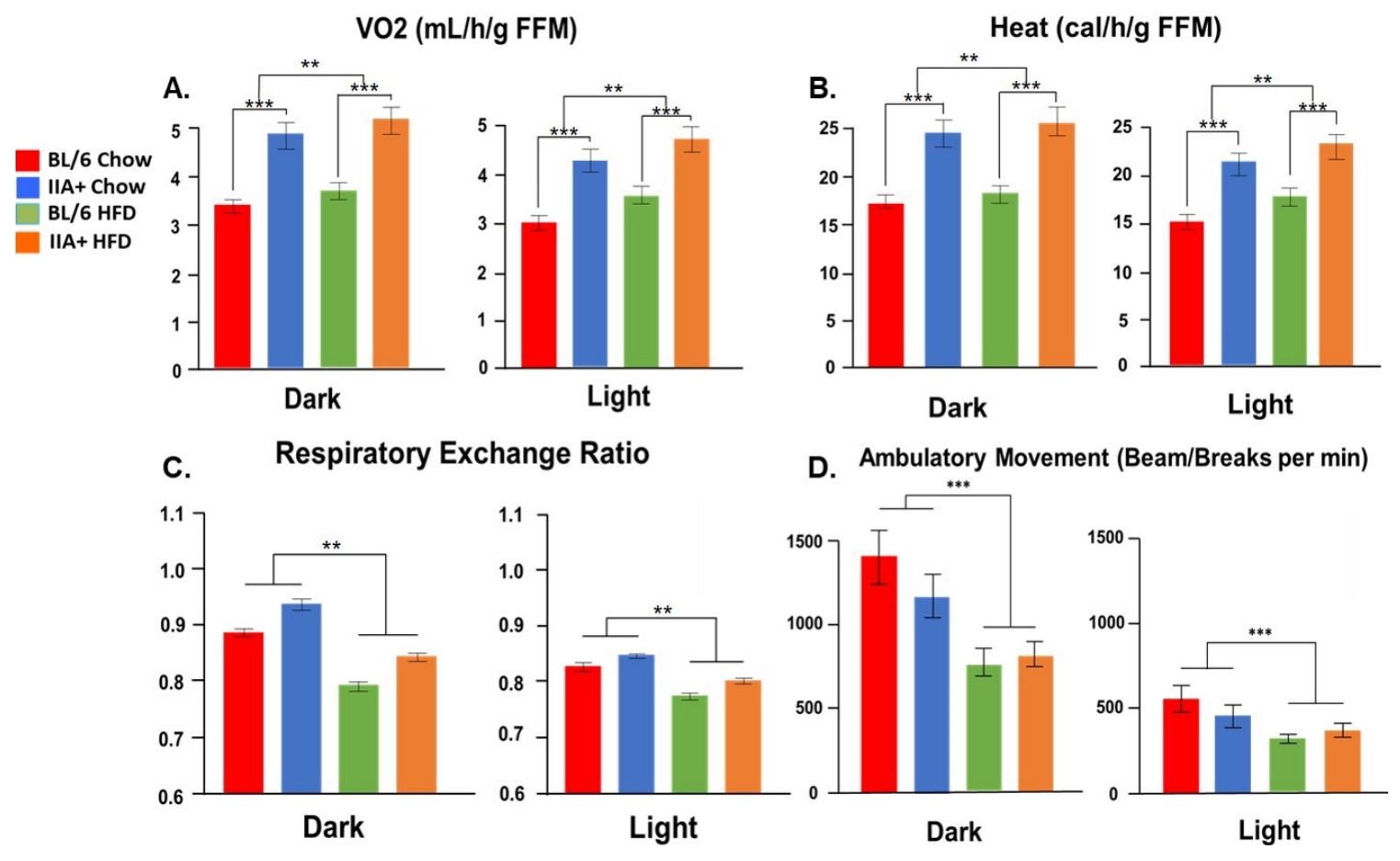

Figure 2-2. Metabolic cage analysis of C57BL/6 and IIA+ mice on HFD

Metabolic analysis and activity of IIA+ mice on the high fat diet. After 10 weeks on the chow diet or HFD, mice were placed individually in CLAM chambers and their metabolism was assessed with respect to fat free mass (FFM). A: VO2 consumption was measured as milliliters per hour of $\mathrm{O} 2$ consumption over a $24 \mathrm{~h}$ period. B: Body heat production was expressed as calories per hour. C: RER was determined for all four groups of mice. D: Activity was determined by the number of beam breaks in both the light and dark cycles and is expressed as ambulatory movement. Each assessment is the average of measurements from 12 animals. Differences between groups were determined by two-way ANOVA, using genotype and diet as two separate factors. ${ }^{*} \mathrm{P}<0.05, * * \mathrm{P}<$ $0.01, * * * \mathrm{P}<0.001$. 


\section{PLA2G2A Improves Insulin Sensitivity}

We examined the glucose and insulin tolerance of these mice after 10 weeks on the diets. Results from the GTT show that the IIA+ mice were able to clear glucose quite rapidly, even after 10 weeks on a high fat diet (Figure 2-3A). However, the BL/6 mice on the high fat diet had reduced glucose clearance and higher levels of blood glucose $2 \mathrm{~h}$ after glucose administration compared with the BL/6 chow-fed mice. There was no effect of the high fat diet on glucose uptake in the IIA+ mice. In Figure 2-3B, the total blood glucose amount over the $2 \mathrm{~h}$ assay is shown. To assess insulin sensitivity, we conducted an ITT. The BL/6 mice on the high fat diet had reduced insulin sensitivity and little change in blood glucose in response to insulin administration. In contrast, the IIA + mice remained insulin sensitive regardless of the diet (Figure 2-3C) and blood glucose remained low $2 \mathrm{~h}$ after insulin administration. The total change in blood glucose level over the $2 \mathrm{~h}$ assay is shown in Figure 2-3D. These data suggest that PLA2G2A enhances whole-body insulin sensitivity.

\section{Plasma Lipid Levels}

Because previous studies have shown that the IIA+ mice have a propensity to develop atherosclerosis, we evaluated the blood lipids of the mice $(15,18)$. The BL/6 mice on the high fat diet had significantly elevated serum cholesterol compared with all other groups. The IIA+ mice on the high fat diet had elevated cholesterol relative to the IIA + chow-fed group, but the overall cholesterol was lower in the IIA+ mice when compared with the BL/6 mice (Figure 2-4A). Plasma triglycerides were lowest in the IIA + chow animals (Figure 2-4B). Triglycerides were highest in the IIA+ mice on the high fat diet, but this elevation was only significant when comparing them to their chow diet counter-part. HDL was greatly elevated in the BL/6 mice on high fat diet, and HDL levels were lower in the IIA+ groups relative to their dietary BL/6 controls (Figure 2-4C). LDL was higher in the IIA+ mice on a high fat diet, although there was great variability among the animals (Figure 2-4D). The increased LDL may reflect the higher triglycerides in the high fat-fed IIA+ mice. Because energy expenditure was higher in the IIA+ mice, we measured the T4 levels in the animals. There was no difference in the T4 between $\mathrm{BL} / 6$ and IIA + mice, although the T4 was lower in both BL/6 and IIA+ animals on the high fat diet (Figure 2-4E). Finally, we measured the triglyceride levels in the liver. The hepatic triglycerides were greatly elevated in the BL/6 mice on the high fat diet, but the lipid levels in the IIA+ mice were only slightly increased (Figure 2-4F).

\section{Tissue Expression of PLA2G2A}

To determine where PLA2G2A was present, we tested various tissues for the expression of PLA2G2A. We observed PLA2G2A mRNA in liver, muscle, eWAT, and BAT (Figure 2-5A through D). The level of PLA2G2A mRNA in the liver was especially high. In the liver and muscle, PLA2G2A mRNA and protein abundance were increased by the high fat diet, indicating that the gene is stimulated by high fat feeding. 
A.

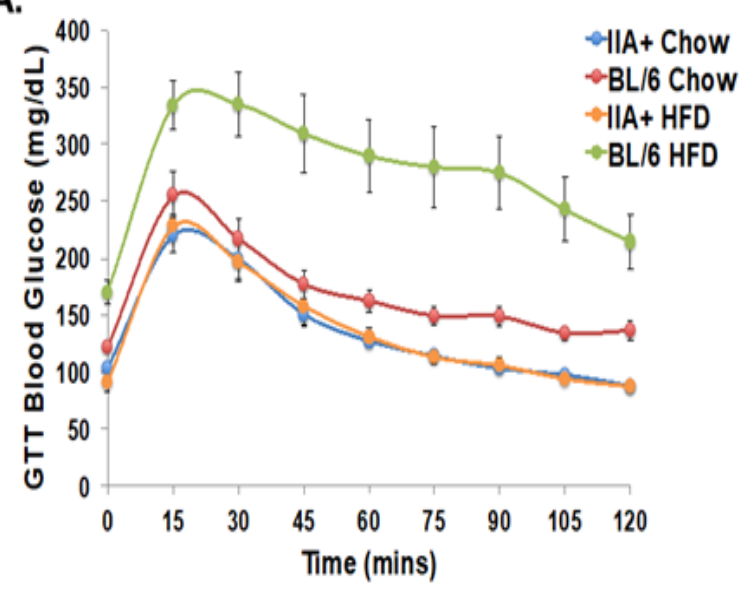

C.

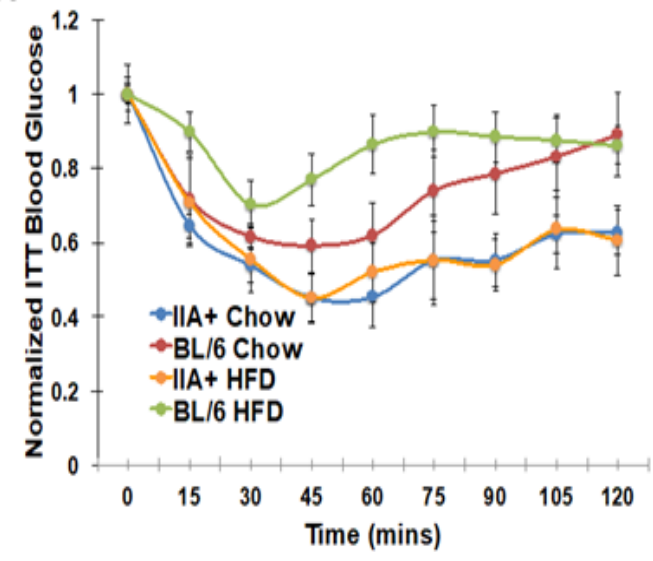

B. 3000

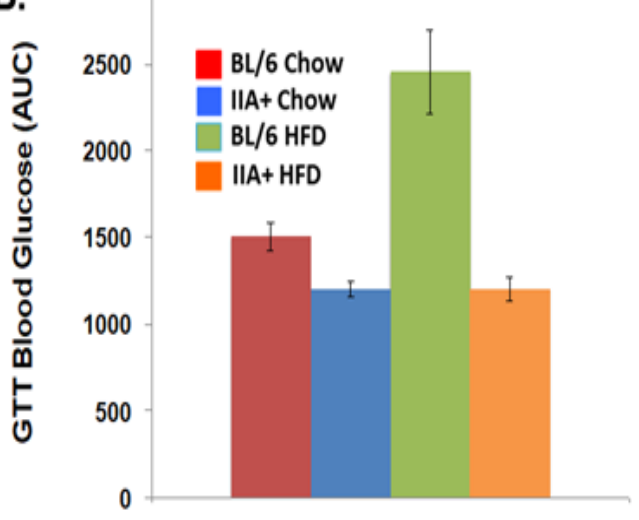

D.

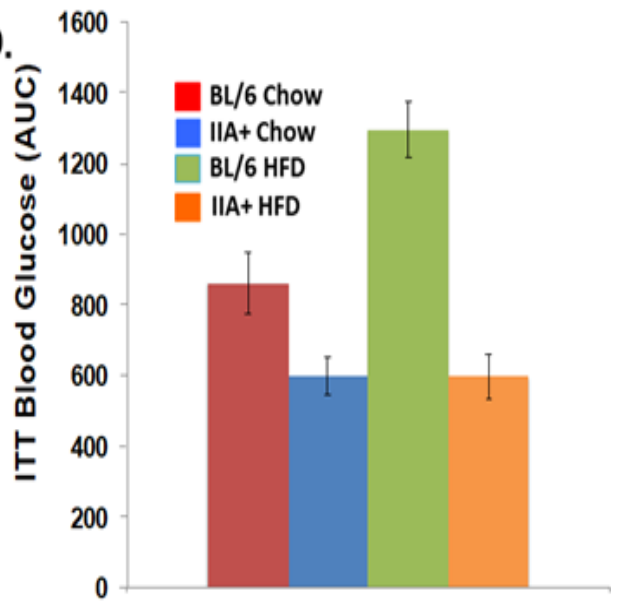

Figure 2-3. PLA2G2As effect on insulin and glucose tolerance

Glucose and insulin tolerance in IIA + mice on the high fat diet. A: GTTs were conducted on all the mice in week 11 of the diet study, as described in the Materials and Methods. The blood glucose was measured at 15 min intervals. Each time point is the average of 11-15 mice. B: The total blood glucose from all time points is shown. C: ITTs were conducted on all the mice in week 12 of the diet study, as described in the Materials and Methods. The blood glucose was measured at 15 min intervals following insulin injection. Each time point is the average of seven to nine mice. D: Total blood glucose for all time points in the ITT is shown. 


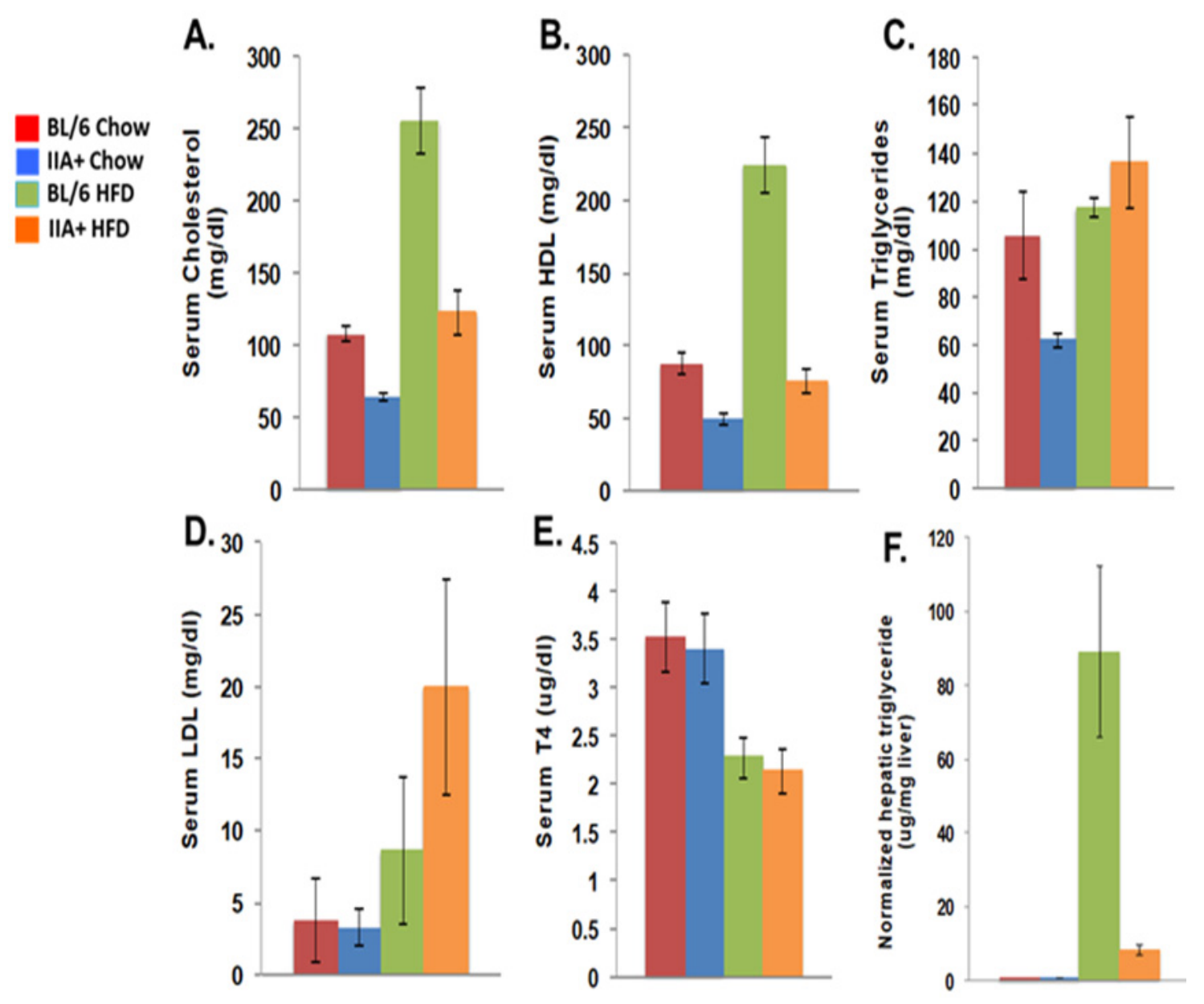

Figure 2-4. Plasma lipid levels of C57BL/6 and IIA+ mice

At the time of euthanization, serum was collected from the mice and blood lipids and thyroid hormone (T4) were measured at the University of Tennessee endocrinology laboratory. A: Cholesterol was measured as milligrams per deciliter of serum. B: Triglycerides were assessed as milligrams per deciliter of serum. C: HDLs were measured as milligrams per deciliter of serum. D: LDLs were measured as milligrams per deciliter of serum. E: T4s were measured as micrograms per deciliter of serum. F: Hepatic triglycerides were measured. Each point is the average of lipids from five animals. 


\section{A. PLA2G2A gene expression}

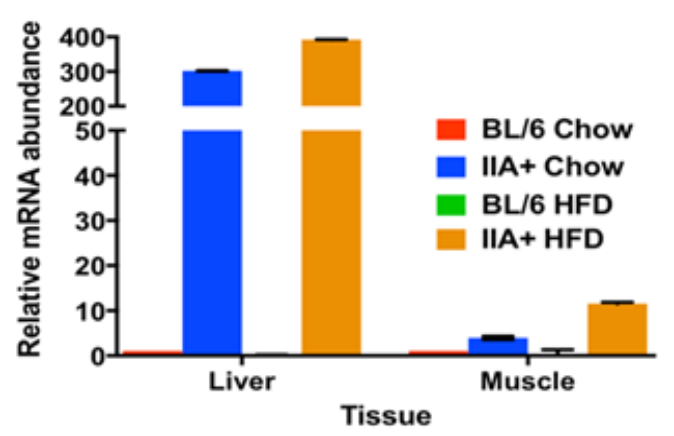

C.

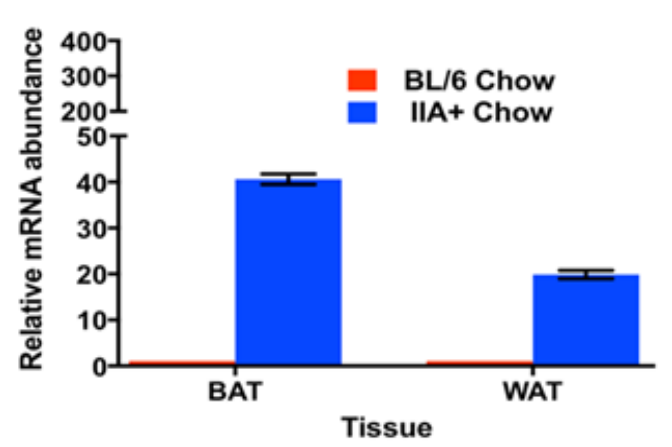

B. PLA2G2A protein expression

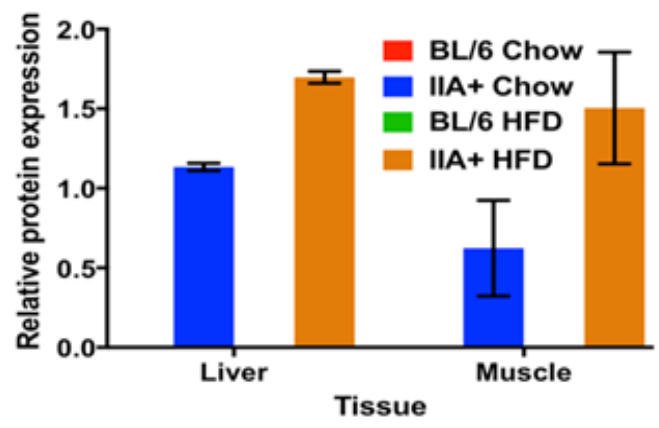

D. PLA2G2A protein expression

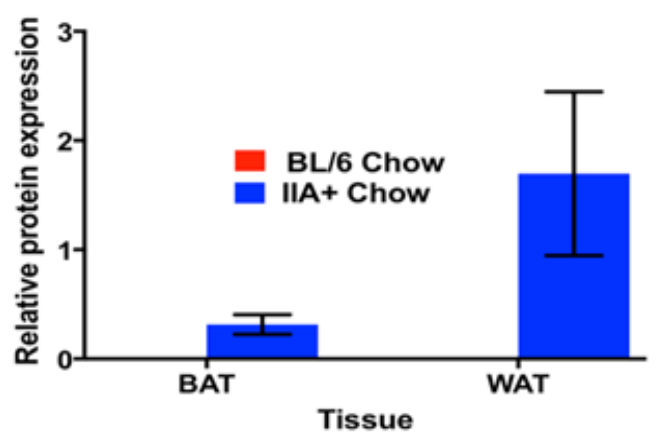

Figure 2-5. Tissue expression of PLA2G2A in IIA+ mice

Expression of PLA2G2A in various tissues. RNA and proteins were collected from mice at the end of the feeding period and PLA2G2A abundance was determined via RT-PCR or Western blot. A: PLA2G2A gene expression from livers and muscle of mice on either chow diet or HFD. B: PLA2G2A protein expression from livers of chow diet or HFD mice. C: PLA2G2A gene expression from BAT and WAT of mice fed only chow diet. D: PLA2G2A protein expression from BAT and WAT of mice fed only chow diet. 


\section{Metabolic Gene Expression in Liver of IIA+ Mice}

To understand the molecular mechanisms underlying the metabolic effects of PLA2G2A, we measured the impact of PLA2G2A on the abundance of various mRNAs encoding metabolic genes in the liver. First, we measured the mRNA abundance of genes involved in fatty acid synthesis and oxidation, including Srebfl, Acaca, and carnitine palmitoyltransferase (Cpt1a). Acaca mRNA levels trended higher in the chow-fed IIA+ mice compared with the IIA+ mice on high fat diet $(\mathrm{P}=0.2871)$, but the levels were not significant. (Figure 2-6A). Surprisingly, the Srebf1 mRNA was reduced in the IIA+ mice (Figure 2-6B). Cptla was elevated in response to the high fat diet in BL/6 mice, but not in the IIA + mice (Figure 2-6C). Next, we measured the hepatic abundance of two genes important for mitochondrial biogenesis, Ppargcla and Sirt1. The mRNA levels of Ppargcla and Sirt1 were higher in the IIA+ mice on the chow diet, but not the high fat diet (Figure 2-6D, E). To identify changes in protein levels, we measured the abundance of hepatic proteins involved in metabolic regulation by Western analyses. The abundance of ACC1 was elevated in both the BL/6 and IIA+ mice on high fat diet (Figure 2-7A). The precursor SREBP-1c protein was elevated in the BL/6 mice in response to the high fat diet, while nuclear SREBP-1c was elevated in the IIA+ mice on the high fat diet (Figure 2-7B, C). We examined inducers of mitochondrial biogenesis, including PPAR $\gamma$ coactivator $1 \alpha($ PGC-1 $\alpha)$ and SIRT1. We did not see any increase in either PGC-1 $\alpha$ or SIRT1 protein in the liver, suggesting that these factors, at least in the liver, do not promote hepatic mitochondrial biogenesis (Figure 2-7D, E).

Because the IIA + mice are more insulin sensitive, we tested to determine whether kinases in the insulin signaling pathway were activated in the IIA + mice. There was increased Akt in the IIA+ mice and there was elevated activation of Akt based on the phospho-Akt(T308) to Akt ratio in the IIA+ mice both on the chow and on the high fat diet (Figure 2-8A, C). In addition, we examined S6K, which is downstream of mTORC1. The data show that there was increased abundance of S6 kinase and phospho-S6 kinase(Thr389), particularly in the IIA+ mice (Figure 2-8B, C). These data suggest that there is enhanced insulin signaling in the IIA + mice.

\section{Thermogenic Protein Expression in BAT}

To determine whether there were changes in other tissues that might account for the increased energy expenditure and heat production in the IIA+ mice, we measured mRNA and protein levels of UCP1, PGC-1 $\alpha$, and SIRT1 in eWAT, BAT, and muscle (Figure 2-9). We did not detect differences in PGC-1 $\alpha$ and SIRT1 abundance in muscle.In eWAT, there was very little gene or protein expression of these metabolic proteins (data not shown). However, in the BAT, Ucp1, Ppargcla, and Sirt1 mRNA levels were greatly increased (Figure 2-9A). We measured the levels of these proteins as well and found that all three were elevated (Figure 2-9B, C). These data suggest that part of the activation of the metabolic rate comes from increased mitochondrial uncoupling and, thus, increased energy dissipation as heat from BAT. 


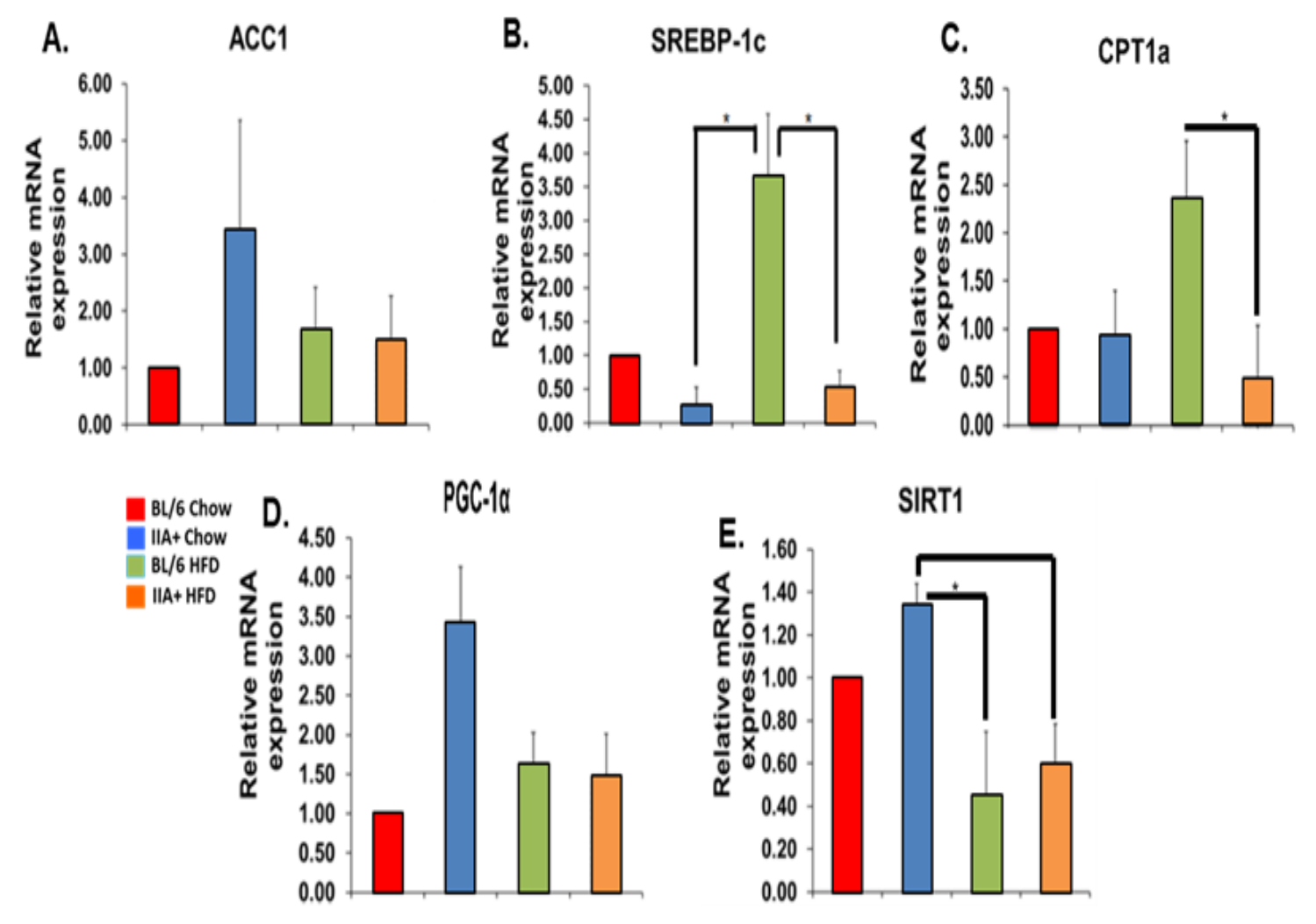

Figure 2-6. Metabolic gene expression in IIA+ mice on HFD

Expression of metabolic genes in livers of IIA+ mice. RNA was collected from the livers at the end of the feeding period. The data are expressed as relative mRNA abundance. $A C C 1$ (A), SREBP-1c (B), CPTla (C), PGC-1 $\alpha$ (D), SIRTl (E). Each point is the average of mRNA from four animals. The data were analyzed by t-test. $* \mathrm{P}<0.05, * * * \mathrm{P}<0.001$. 

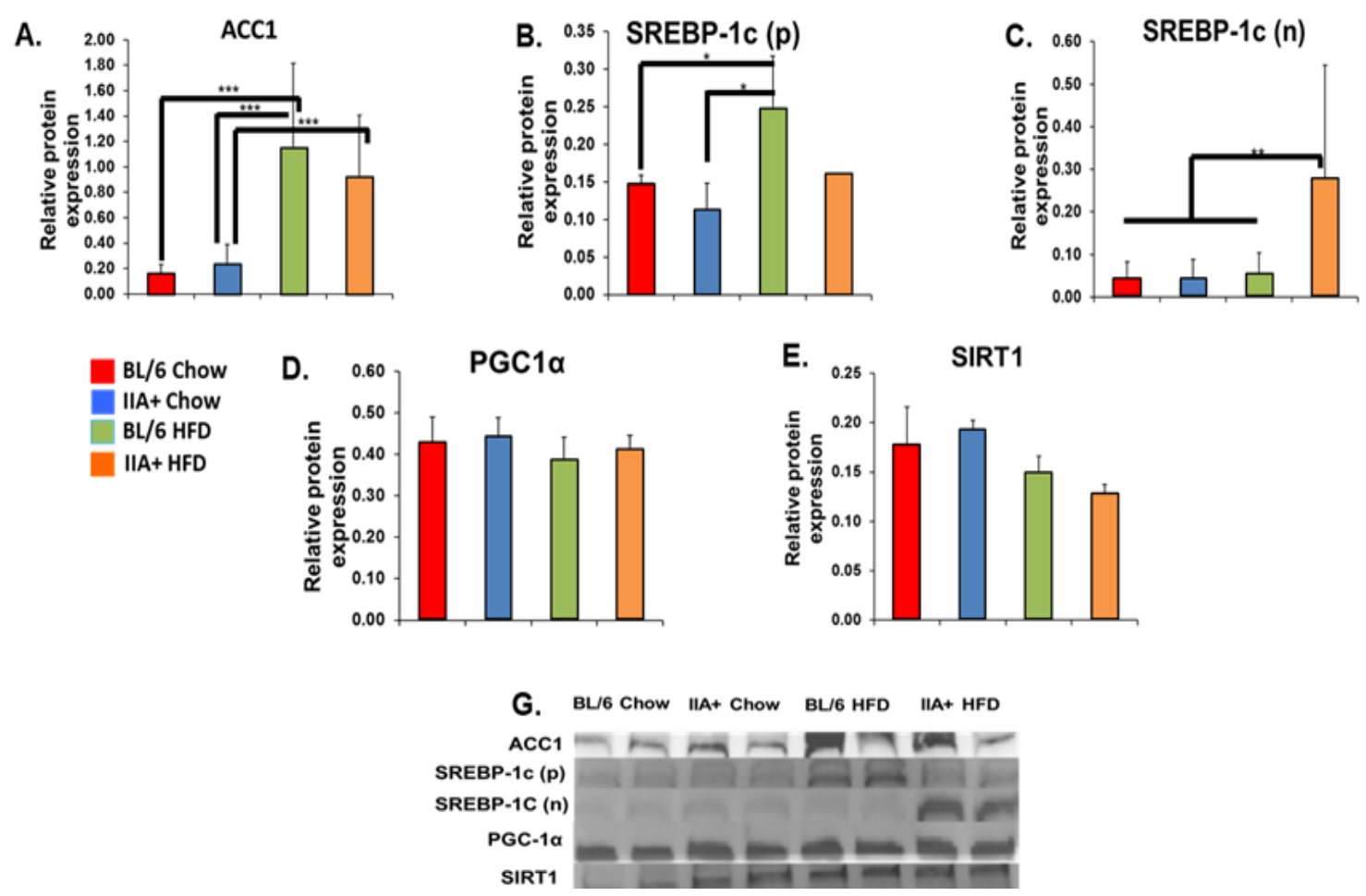

Figure 2-7. Metabolic protein expression in IIA+ mice on HFD

Changes in metabolic factors in livers of IIA + mice. Proteins were collected from the livers at the end of the feeding period and the abundance of various proteins was analyzed by Western blot. Representative Western blots are shown and the data was quantified. ACC1 (A), precursor SREBP-1c (B), nuclear SREBP-1c (C), PGC-1 $\alpha$ (D), SIRT1 (E), representative blots are shown $(\mathrm{F})$. The data are expressed as relative protein abundance. Each point is the average of Western data blot from four animals. The data were analyzed by t-test. $* \mathrm{P}<0.05, * * \mathrm{P}<0.01, * * * \mathrm{P}<0.001$. 
A.

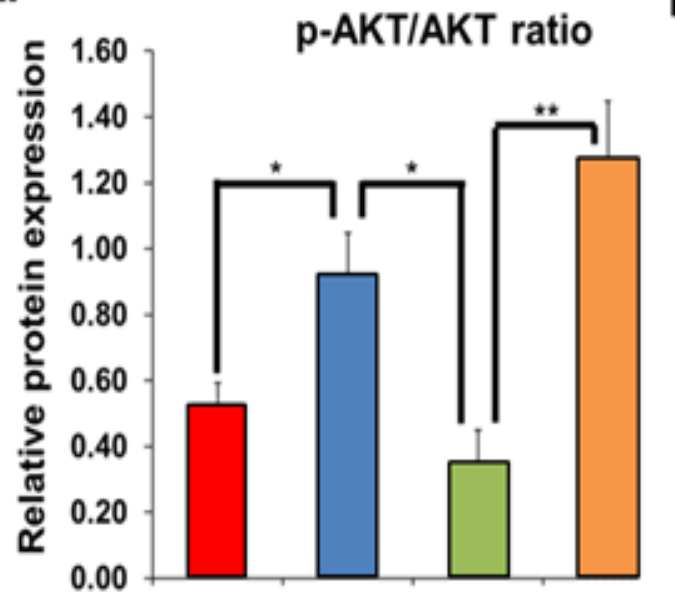

B.

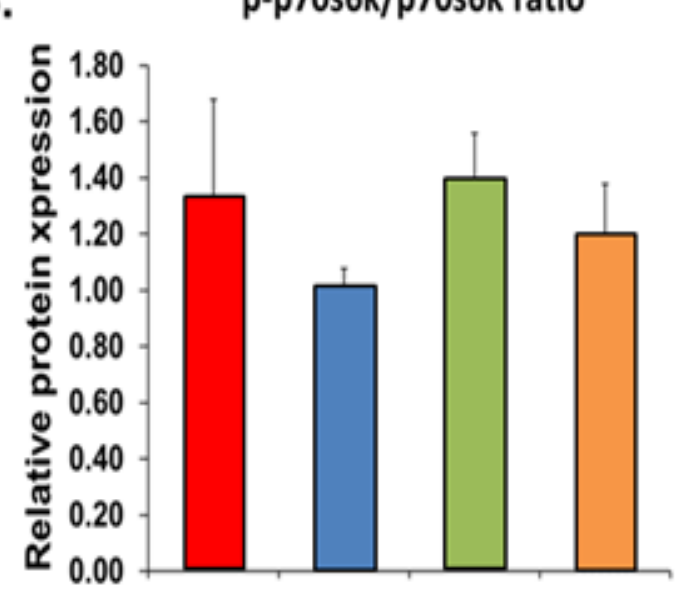

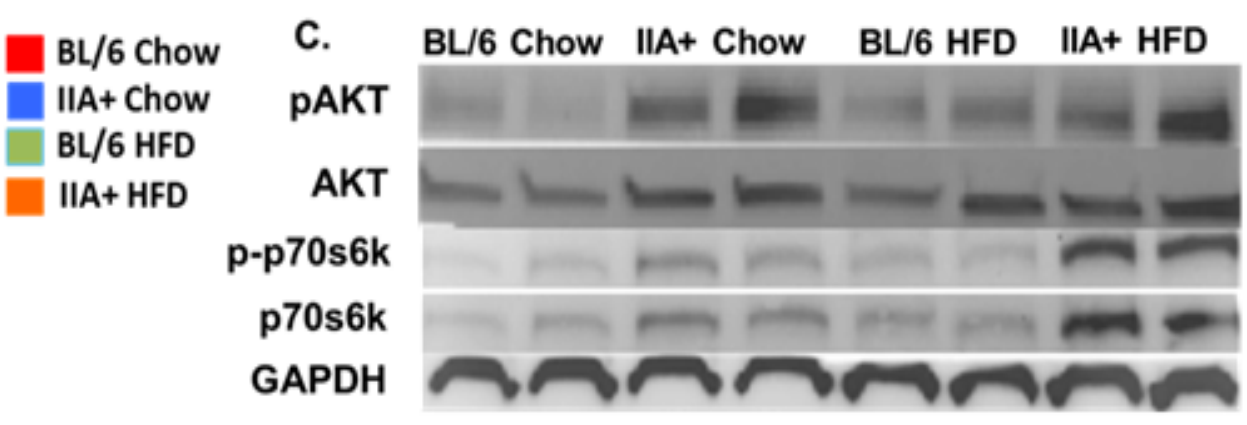

Figure 2-8. Hepatic expression of insulin signaling markers

Alterations in insulin signaling pathways in IIA+ mice. Proteins were collected from the livers at the end of the feeding period and the abundance of various kinases was analyzed by Western blot. Kinase abundance was measured and the ratio of phospho-kinase to kinase is shown. pAkt/Akt (A), pS6K/S6K (B), representative Western blots are shown (C). Each point is the average of proteins from four animals. The data were analyzed by $\mathrm{t}-$ test. $* \mathrm{P}<0.05, * * \mathrm{P}<0.01$. 

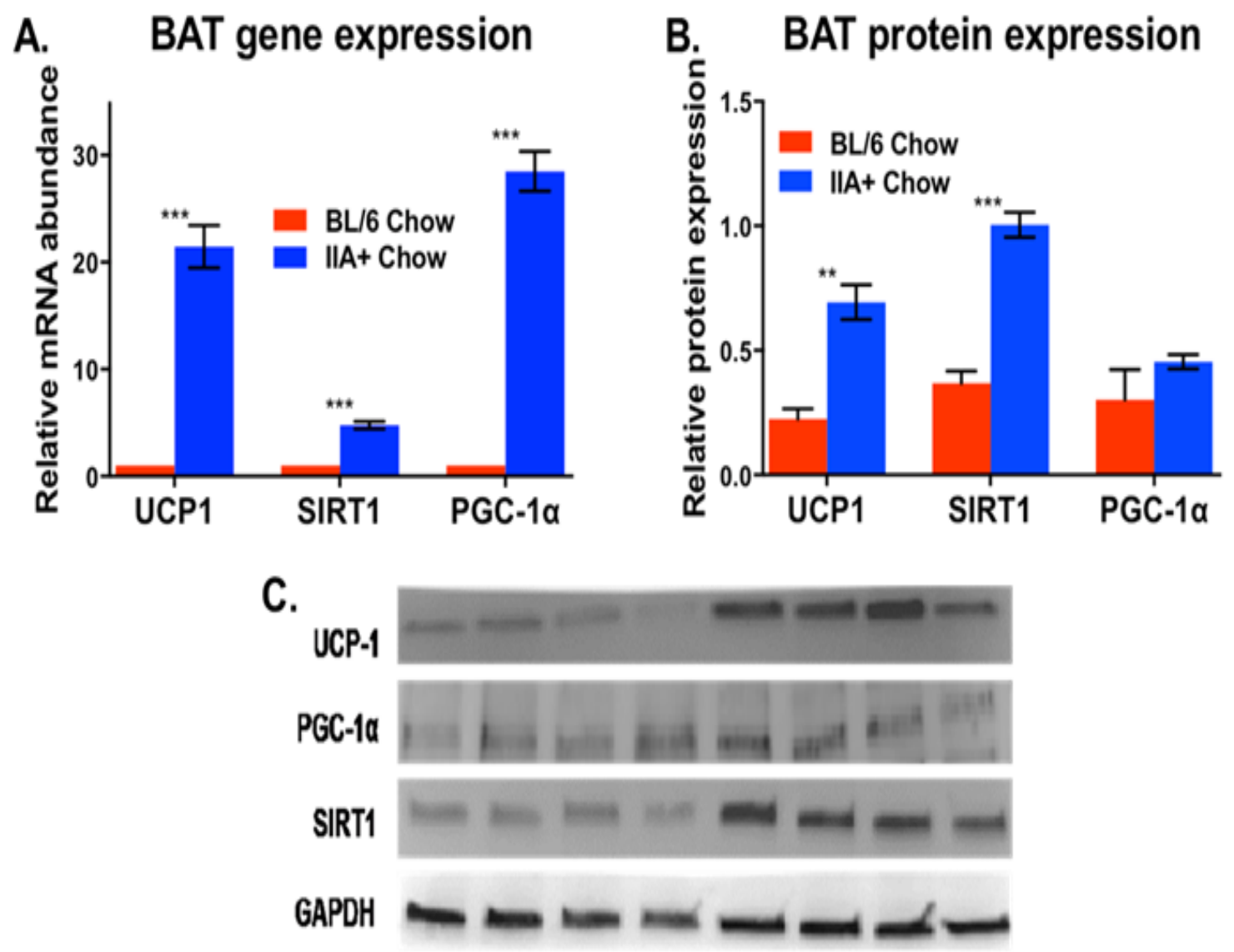

Figure 2-9. Thermogenic protein expression induced in IIA+ BAT

Increased mitochondrial uncoupling in IIA + mice on chow-fed diet. RNA and proteins were collected from the BAT of BL/6 and IIA+ mice after a 10 week feeding period. A: The relative mRNA levels of Ucp1, Sirt1, and Ppargc1a are shown. B: The relative protein abundance of UCP1, SIRT1, and PGC-1 $\alpha$ are shown. C: The Western blots from the BAT are shown. Each point is the average of mRNA/protein from four animals. Data were analyzed by t-test. $* \mathrm{P}<0.05,{ }^{*} * \mathrm{P}<0.01, * * * \mathrm{P}<0.001$. 


\section{Discussion}

Elevated levels of the PLA2G2A are associated with inflammation and cardiovascular disease (117). Here, we investigated the metabolic response of IIA+ mice to a high fat diet. My data indicate that PLA2G2A provided protection from the deleterious effects of high fat diets. First, the IIA+ mice did not gain weight or accumulate fat mass in response to the high fat diet and, additionally, remained insulin sensitive and glucose tolerant. Second, the IIA+ mice had elevated energy expenditure and heat generation, likely due to the induction of mitochondrial uncoupling in BAT. My results demonstrate that PLA2G2A enhances insulin responsiveness and overall energy utilization.

The focus of many previous studies on PLA2G2A has been on the regulation of cholesterol levels and the impact of PLA2G2A on atherogenesis $(18,19,118)$. sPLA2 enzymes, including PLA2G2A, PLA2G3, and PLA2G5, are pro-atherogenic due to their ability to promote the oxidation of LDL and HDL particles and to increase the formation of lipid-laden foam cells from macrophages (3). Introduction of PLA2G2A-expressing bone marrow cells into $\mathrm{Ldlr}-/$ - mice increased the formation of atherosclerotic lesions (19). Likewise, PLA2G3 has been found to enhance the development of atherosclerosis and small pro-atherogenic LDL particles (74). PLA2G2A promotes atherogenesis in IIA+ mice fed a high cholesterol diet (15) and IIA+ mice had hepatic cholesterol accumulation on an atherogenic diet (17). My experimental design differed from previous studies in that the diets used contained significantly greater amounts of fat, but less cholesterol. Consistent with the mice on atherogenic diets, we found that there was reduced blood cholesterol in the IIA+ mice (Figure 2-4A). The chow-fed IIA+ mice also had lower HDL and reduced serum triglycerides. The lower HDL raises the possibility that the mice have diminished reverse cholesterol transport. The elevated triglycerides in the IIA+ mice on the high fat diet are likely associated with increased LDL particles (Figure 2-4D). The lower HDL and elevated LDL may contribute to the atherosclerosis observed in the IIA+ mice (15). A trend toward elevated VLDL/LDL particles was previously reported in IIA+ mice on an atherogenic diet (15). The IIA+ mice also had reduced Cptla mRNA, suggesting less hepatic fatty acid oxidation (Figure 2-6C). In addition, the IIA+ mice had higher levels of ACC1 and nuclear SREBP-1c, which may also contribute to the hyperlipidemia (Figure 2-7). Overall, my data are consistent with previous observations of changes in blood lipids in the IIA+ mice.

An unexpected finding of these experiments was that the IIA + mice did not gain weight or accumulate fat mass on the high fat diet. Only the BL/6 mice on the high fat diet accumulated fat. The IIA+ mice were smaller than the BL/6 mice and consumed similar kilocalories per gram of body weight over the 10 -week period. The increased energy expenditure was likely the major cause of the reduced weight gain by the IIA+ mice. Increased energy expenditure was not due to increased activity in the IIA + mice, as they were not more active than the BL/6 mice (Figure 2-2D). Elevated thyroid hormone concentrations were not responsible (Figure 2-4E). In the liver, key regulators of proteins involved in fatty acid oxidation, such as PGC-1 $\alpha$ and SIRT1, were not elevated. The transcriptional events that specify a brown adipogenic program have been well- 
characterized. PGC-1 $\alpha$ and the transcription factor PR domain containing 16 (PDRM16) directly regulate BAT induction, and SIRT1 may promote adipose tissue browning as well (119-121). We observed increased expression of UCP1, PGC-1 $\alpha$, and SIRT1 in the $\mathrm{BAT}$ of the IIA+ mice, suggesting that PLA2G2A promotes mitochondrial uncoupling in BAT (Figure 2-9).

It was previously discovered that lipoproteins modulated by sPLA2 were more susceptible to lipid peroxidation, resulting in the production of oxidized lipoproteins and the by product, 4-hydroxy-2-nonenal (4-HNE). The 4-HNE can induce mitochondrial uncoupling through covalent modification of UCPs and adenine nucleotide translocase (ANT), an ATP exporter (122). Given the increases we have observed in overall brown adipogenic programming through induction of PGC- $1 \alpha$ and SIRT1, as well as induction of UCP1 itself, in the IIA+ mice, a possible explanation is that overexpression of PLA2G2A enhanced lipid peroxidation and, therefore, 4-HNE. The 4-HNE could activate mitochondrial uncoupling through its stimulatory actions on proton conductance.

One of the many fatty acids released by sPLA2 activity is arachidonic acid, a necessary precursor for biosynthesis of a diverse group of eicosanoids and other signaling molecules involved in the inflammatory response (123). However, it was recently discovered that arachidonic acid alone can also directly activate the mitochondrial proton leak caused by UCP 1 in both beige and brown adipocytes through experiments using inner mitochondrial matrix mitoplasts (124). Moreover, a similar study found that UCP1 can be controlled by fatty acid products generated from phospholipid hydrolysis by cytosolic PLA2 (125). The IIA+ mice on chow and high fat diets had elevated VO2 (Figure 2-2A) and heat dissipation (Figure 2-2B). This may be due to enhanced release of arachidonic acid or other long chain fatty acid (LCFA) signaling molecules by sPLA2. This LCFA then migrates to BAT and induces mitochondrial uncoupling through UCP1's LCFA anion $/ \mathrm{H}+$ symporter activity.

Because PLA2G2A is frequently associated with inflammation and rheumatoid disease, we anticipated that the IIA+ mice would be insulin resistant, as inflammation has been frequently linked with insulin resistance $(126,127)$. However, another unexpected observation was that the IIA+ mice were much more sensitive to insulin, as demonstrated by both the GTT and the ITT (Figure 2-3). Furthermore, the hepatic insulin signaling pathways were clearly activated, as shown by the increased abundance and phosphorylation of Akt (Figure 2-8). One possibility is that as the IIA+ mice were refractory to high fat diet-induced obesity, the insulin sensitivity was secondary to the different obesity states. Previous work found that cholesterol accumulated in the liver of IIA+ mice on an atherogenic diet (17). The livers of the BL/6 mice on high fat diet contained much more triglyceride than those of all other groups (Figure 2-4F). We observed a slight increase in hepatic triglycerides in the IIA+ mice on high fat diet compared with the chow groups. The attenuated accumulation of triglycerides in the IIA+ mice may provide an additional reason as to why the IIA+ mice on high fat diet are not insulin resistant. 
Recent studies have found that several sPLA2 isoforms possess different and tissue-specific roles in obesity and metabolic dysfunction. Hui and colleagues reported that the knockout of PLA2g1b provided protection against diet-induced obesity and insulin resistance (128). PLA2 $\mathrm{g} 1 \mathrm{~b}$ is secreted into the intestinal lumen from the pancreas and the protection arose from reduced lysophosphatidylcholine absorption. The PLA2g1b knockout mice also had elevated hepatic fatty acid oxidation, indicating that the livers relied more on fatty acids for energy $(35,40)$. Addition of lysophosphatidylcholine to isolated hepatocytes directly reduced mitochondrial oxidation of fatty acids (129). Work from Murakami and colleagues examined the interplay of Pla2g5 and Pla2g2e in the adipose tissue with respect to metabolic actions (69). They found that PLA2g5 knockout mice displayed insulin resistance and hyperlipidemia, and were increasingly prone to diet-induced obesity, suggesting that PLA2g5 provides protection from obesity. In the same study, it was also discovered that knocking out PLA2g2e in mice decreased hyperlipidemia and diet-induced obesity, and improved overall liver health, indicating that PLA2g2e may promote obesity (69). A metabolic role for PLA2g2a was investigated in rats (50). With a high fat diet, the levels of PLA2g2a were elevated 20-fold in rat white adipose tissue (WAT) (50). In concurrence with these studies, our lab previously reported that BALB/c mice on a high fat diet had increased Pla $2 g 2 a$ mRNA in the liver and that the addition of oleic acid to rat hepatocytes increased PLA2g2a (46). In rats, chronic administration by gavage of the PLA2g2a inhibitor, KH064, protected against diet induced weight gain and reduced fasting glucose concentrations (50). The authors proposed that PLA2g2a inhibition improved WAT function via stimulating lipolysis, thereby decreasing fat stores in WAT (50). These results differ from my observations that elevated PLA2G2A activity enhances energy expenditure in BAT and overall insulin sensitivity. It may be that the results in the rat study stem from an impairment of intestinal lipid absorption, thereby reducing the impact of the high fat diet, as Iyer et al. (50) observed increased fat in the feces of the KH064 treated rats. In addition, Iyer et al did not measure food intake with KH064 administration. It is clear from multiple studies that the various secretory phospholipases can have isoform-specific and tissue-specific effects. For example, Boilard et al. (13) reported that PLA2g5 has an anti-inflammatory role that counteracts the inflammation mediated by PLA2g2a in rheumatoid arthritis. Therefore, the role of PLA2G2A and other sPLA2 isoforms in metabolism requires further investigation.

In summary, we have found that PLA2G2A improves insulin sensitivity and provides resistance to high fat diet-induced weight gain in mice. Furthermore, PLA2G2A increases the energy expenditure of the mice independent of activity and diet, partly through UCP1 and induction of BAT. We have identified a previously undescribed role of this secretory phospholipase in the modulation of BAT and metabolism. Future studies will determine the mechanisms underlying this phenomenon. 


\section{CHAPTER 3. PLA2G2A ENHANCES THE METABOLIC RATE AND INCREASES GLUCOSE UTILIZATION IN RESPONSE TO THYROID HORMONE*}

\section{Introduction}

Obesity is a metabolic condition marked by excessive fat accumulation and a high body-mass index. Obesity is associated with life-threatening health complications such as cardiovascular disease, diabetes and high blood pressure. Recently, the beiging of white adipose tissue (WAT), which is the conversion of adipose tissue from an energy storing to energy-dissipating tissue, and the activation of brown adipose tissue (BAT) have emerged as potential therapeutic targets to treat obesity. BAT has a thermogenic role in rodents and human infants (130). It generates heat by uncoupling oxidative phosphorylation to dissipate the protons required for ATP generation through uncoupling protein-1 (UCP1) (131). Increased amount and activity of BAT have been associated with improved glucose and triglyceride clearance (132), reduced fat mass, and enhanced insulin sensitivity (133). Furthermore, beige-like adipose tissue has elevated mitochondrial activity and counteracts high fat diet-induced weight gain (134). While it was generally accepted that humans contained little to no BAT following infancy, studies using fluorodeoxyglucose positron emission tomography (FDG-PET) revealed that the tissue does exist in humans (135).

Triiodothyronine (thyroid hormone; T3) is a major regulator of metabolic processes $(136,137)$, including hepatic cholesterol production and disposal, adipose tissue lipolysis and basal metabolic rate (138). With respect to adipose tissue, T3 status is a major determinant of BAT activity and the beiging of WAT. A recent study found that hyperthyroidism in mice increased BAT mass and activity, while hypothyroid mice had diminished BAT activity (139). Interestingly, both hypo- and hyperthyroid mice displayed an enhanced induction of a thermogenic gene profile in inguinal WAT, indicating WAT thermogenesis may compensate for the reduced BAT activity in a hypothyroid state. Similar effects were observed in hyperthyroid rats. Although the hyperthyroid rats had elevated food intake, they gained less weight, most notably due to induction of BAT thermogenic genes including PR domain containing 16 (PRDM16), Cell Death-Inducing DFFA-Like Effector A (CIDEA), and UCP1 in the inguinal and epididymal WAT (140). Perhaps most significantly, T3 levels positively correlate with markers for the beiging of WAT in humans (140), which suggests WAT beiging may account for the increased energy expenditure seen in hyperthyroidism $(141,142)$.

* Reprinted from final submission with permission. Kuefner M.S., Deng X., Stephenson E.J., Pham K., Park E.A. Secretory phospholipase A2 group IIA enhances the metabolic rate and increases glucose utilization in response to thyroid hormone. FASEB J. 2019; 33:738-749 https://doi.org/10.1096/fj.201800711R (52). 
Previously, PLA2G2A was investigated for its involvement in the development and progression of inflammation and atherosclerosis $(6,111)$. However other than our work, its role in metabolism has not been studied which is significant given the emerging information that several sPLA2s have roles in the regulation of obesity, hepatic steatosis, hyperlipidemia and insulin signaling (143). Our lab's previous findings showed that acute treatment with T3 inhibits PLA2G2A gene expression in the livers of rats and mice (46). However, the effects of PLA2G2A in altered thyroid states on energy expenditure and insulin sensitivity are not known. C57BL/6 (BL/6) mice do not express PLA2g2a due to a frameshift mutation in exon 3. The human PLA2G2A gene was introduced into C57BL/6 $(\mathrm{BL} / 6)$ to create PLA2G2A expressing (IIA+) mice $(15,16)$. In Chapter 2, we showed that IIA + mice had increased total energy expenditure, improved insulin sensitivity, and were protected against diet-induced obesity (51). This suggests PLA2G2A may play an important role in metabolic regulation.

In this study, we assessed the metabolic differences between BL/6 and IIA+ mice in response to hypothyroidism or hypothyroidism followed by $\mathrm{T} 3$ replacement. We found that the IIA+ mice had greater food intake but accumulated less fat and overall body weight regardless of the thyroid status. The chow and T3 treated IIA + mice had elevated metabolic rates without differences in physical activity. Markers of mitochondrial uncoupling were enhanced in both white and brown adipose depots of the IIA+ mice. Finally, we found that T3 treatment sensitized the IIA+ mice to insulin, lowered their blood glucose, and pushed fuel utilization to almost exclusively carbohydrates when they were active. This study has identified a novel metabolic role of PLA2G2A in response to variations in thyroid hormone status and activation of BAT.

\section{Materials and Methods}

\section{PLA2G2A Expressing C57BL/6 Mice (IIA+)}

The IIA + mice, which express the human PLA2G2A gene under the regulation of the human promoter, were provided by Dr. Eric Boilard at the Universite Laval (CHUL), Quebec, Canada (114). Male mice heterozygous for the PLA2G2A gene were bred with C57BL/6 (BL/6) females. For these experiments, male BL/6 and IIA+ mice were used. Mice were housed according to genotype and placed on their respective diets 8 weeks after birth. About the dietary groups, we fed $11 \mathrm{BL} / 6$ and 9 IIA + mice chow diet for 10 weeks, put $10 \mathrm{BL} / 6$ and 7 IIA+ mice on a hypothyroid regimen for 10 weeks, and had 10 BL/6 and 7 IIA+ mice on the hypothyroid regimen for 5 weeks, followed by thyroid hormone treatment for 5 weeks. Two or three littermates per cage of the same genotype and diet were housed with a constant light and dark phase of 12 hours at $20-23^{\circ} \mathrm{C}$. All animal procedures were approved by the UTHSC Institutional Animal Care and Use Committee. 


\section{Diets and Food Intake}

The chow diet was supplied by Research Diets Inc (AIN-76A rodent diet). The mice were made hypothyroid by providing the AIN-76A diet without added iodine, along with $0.15 \%$ propylthiouracil (PTU) and $0.02 \%$ methimazole (MMI) in the drinking water for 10 weeks (144). For the thyroid hormone treatment groups, mice were put on the hypothyroid regimen for 5 weeks, then switched to the AIN-76A rodent diet without PTU or MMI in the drinking water. They were injected subcutaneously with thyroid hormone (40ng T3/g body weight every other day). The mice were given free access to food and water throughout the study. The food was weighed at weekly intervals and the kcal consumption was calculated based on grams of food eaten, the calorie density of the food and the number of mice per cage.

\section{Body Composition}

Mice were weighed and total fat and fat-free mass was determined weekly by EchoMRI-1100 (51).

\section{Glucose and Insulin Tolerance Tests}

Following 6-hour fasts, baseline blood glucose concentrations were determined in blood collected from a small tail incision using a hand-held glucometer (AccuCheck). Dglucose in PBS (2 mg/kg fat-free mass; GTT) or insulin $(0.75 \mathrm{U} / \mathrm{kg}$ fat-free mass; ITT) was injected intraperitoneally and blood glucose concentrations measured at 15, 30, 45, $60,75,90,105$ and 120 minutes post-injection. The data are presented as blood glucose levels versus time and the area under the glucose curve.

\section{Metabolic Cages}

After 10 weeks of diet, mice were individually housed in the CLAMS for 7 days. They were maintained on either chow, hypothyroid, or thyroid hormone replacement regimens. Total energy expenditure was determined using indirect calorimetry at $25^{\circ} \mathrm{C}$. $\mathrm{VO} 2$ and total energy expenditure were expressed relative to fat-free mass. Rates of fatty acid and carbohydrate oxidation were calculated using equations established by Péronnet and Mariscotti that assume protein oxidation is negligible (145). The number of infrared beam breaks along the $\mathrm{X}$ and $\mathrm{Y}$ axes of the cage were counted as a measure of physical activity.

\section{Western Blots}

Mice were fasted 1 week after being housed in CLAMS chambers. Prior to sacrifice, the mice were fasted 6 hours and liver, BAT, epididymal white adipose tissue 
(WAT), quadriceps muscles, and serum were collected. Western blots were performed according to the protocol described previously (51). Across the various tissues, the following proteins were measured: p-p70S6K(Thr389), (Cell Signaling Technology 108D2); p70S6K, (Cell Signaling Technology 9202); UCP2, (Cell Signaling Technology D105V); GAPDH, (Cell Signaling Technology 14C10); PLA2G2A, (Abcam AB23705); Sirtuin 1 (SIRT1), (Life Technologies A21993); SREBP-1c, (BD Pharmingen 5351581), uncoupling protein 1 (UCP1), (Cell Signaling Technology D9D6X); Peroxisome proliferator-activated receptor gamma coactivator 1-alpha (PGC-1 $\alpha$ ), (Novus Bio NBP104676); fatty acid transport protein-1 (FATP1), (Abcam ab69458); Glucose transporter type 4 (GLUT4), (Cell Signaling Technology 1F8); PR domain containing 16 (PRDM16), (Abcam ab106410); Early B Cell Factor 2 (EBF2), (R\&D Systems AF7006). In all tissues, GAPDH was used as the control. Western blots statistics were performed using the Graphpad Prism 7.0 program. Data were presented as the mean results of 4-5 replicate experiments \pm the standard error. The data were further analyzed by Student's two tailed t-test or ANCOVA for CLAMS analyses, using lean mass as the covariate and each measurement as the dependent variable. P-values $<0.05$ were considered statistically significant.

\section{Thyroid Hormone Levels}

Serum T3 levels were determined by the University of Tennessee Endocrinology laboratory (46).

\section{Primary Culture of Adipocytes}

Stromal vascular fraction (SVF) was collected from the white (epididymal and subcutaneous) and brown (interscapular) adipose tissue of 4- 6 week old BL/6 and IIA+ mice using an adipocyte isolation and differentiation protocol described previously (146). Tissue was collected and digested in a solution containing collagenase, phosphatebuffered saline (PBS), and calcium chloride $(\mathrm{CaCl} 2)$, an activator of collagenase. After digestion, the tissue suspension containing the SVF was filtered through a $100 \mathrm{uM}$ cell strainer, centrifuged in a benchtop centrifugation, washed twice with PBS, and plated in collagen-coated dishes. Cells were maintained in Dulbecco's modified eagle's medium nutrient mixture F-12 Ham (DMEM/F12) containing 10\% fetal bovine serum (FBS), and an antibiotic solution containing penicillin and streptomycin $(\mathrm{P} / \mathrm{S})$. After the SVF cells reached complete confluence, the SVF cells which contained pre-adipocytes were switched to an induction cocktail for adipocyte differentiation. The induction cocktail was composed of rosiglitazone, dexamethasone, 3-isobutyl-1-methylxanthine (IBMX) and insulin (147). After 2 days of induction, the cells were incubated in the maintenance media containing DMEM/F12, 10\% FBS, $1 \mathrm{uM}$ insulin, $1 \mathrm{nM} \mathrm{T3}$, and $1 \mathrm{uM}$ rosiglitazone. The cells were maintained in this media for 7 days with the media being replaced every other day. To assess the effect of $\mathrm{T} 3$ in the differentiated adipocytes, WAT and BAT cells were incubated in DMEM/F12 containing 10\% charcoal-stripped serum for 24 hours. Then the cells were incubated for $16 \mathrm{~h}$ in DMEM/F12 containing 
$10 \%$ charcoal-stripped serum with or without $1 \mathrm{nM}$ of T3. Adipocytes were harvested and the mRNA and protein levels of markers for mitochondrial uncoupling (PGC-1 $\alpha$, UCP1, SIRT1) were assessed. Adipose tissues from 4-5 mice per group (BL/6 or IIA+) were pooled to obtain sufficient SVF cells for adipocyte differentiation.

\section{Results}

\section{PLA2G2A Decreases Body Weight and Fat Mass}

The first experiments were designed to measure changes in body composition and food intake over a 10 week period in the BL/6 and IIA+ mice in response to a chow diet, hypothyroidism, or hypothyroidism with 5 weeks of T3 replacement. To verify the thyroid status in the hypothyroid or T3 treated mice, we measured total T3 in the serum at the time of sacrifice (Figure 3-1). We found that hypothyroidism markedly reduced weight gain and lean mass in the BL/6 and IIA+ mice as early as 1 week after being given PTU (Figure 3-2A, B). However, when the hypothyroid mice were treated with T3 after 5 weeks of PTU, their weight and lean mass immediately rose, matching or exceeding weight gain observed in the BL/6 and IIA+ mice on the chow diet. Adiposity was significantly lower in the IIA+ mice compared to their BL/6 counterparts, regardless of their thyroid status (Figure 3-2C). However, T3 treatment did increase adiposity in the IIA + mice compared to the IIA + chow group. Despite greater body weight and fat mass of the BL/6 mice, the IIA+ mice consumed more calories than the BL/ 6 mice independent of their thyroid status (Figure 3-2D).

\section{PLA2G2A Increases Energy Expenditure and Carbohydrate Utilization with T3}

Next, we examined differences in energy expenditure, substrate utilization, and physical activity. The IIA + mice on chow diet (IIA + chow) and treated with T3 (IIA+ T3) displayed elevated oxygen consumption (VO2) and carbon dioxide production (VCO2) compared to the $\mathrm{BL} / 6$ mice. The increased VO2 and VCO2 was particularly noticeable in the dark (active) cycle (Figure 3-3A, B). Elevations in VO2 and VCO2 did not occur in the hypothyroid IIA+ mice indicating that PLA2G2A exerts metabolic effects only in the presence of T3 (Figure 3-3A, B). Similarly, total energy expenditure was enhanced in the IIA + chow and IIA + T3 mice. However, expression of PLA2G2A did not increase total energy expenditure in the hypothyroid mice (Figure 3-3C). Respiratory exchange ratio (RER) was unchanged across all groups when the mice were at rest (light cycle). However, when the mice were active (dark cycle), the IIA+ T3 group had an RER around 1.0, indicating almost exclusive use of carbohydrate as their fuel source (Figure 3-3D). Moreover, fatty acid and carbohydrate oxidation were both elevated in the IIA+ T3 mice during the light cycle compared to their BL/ 6 counterparts, supporting increased total energy expenditure and suggestive of enhanced energy requirements. This effect was completely reversed in the dark cycle, as carbohydrate 


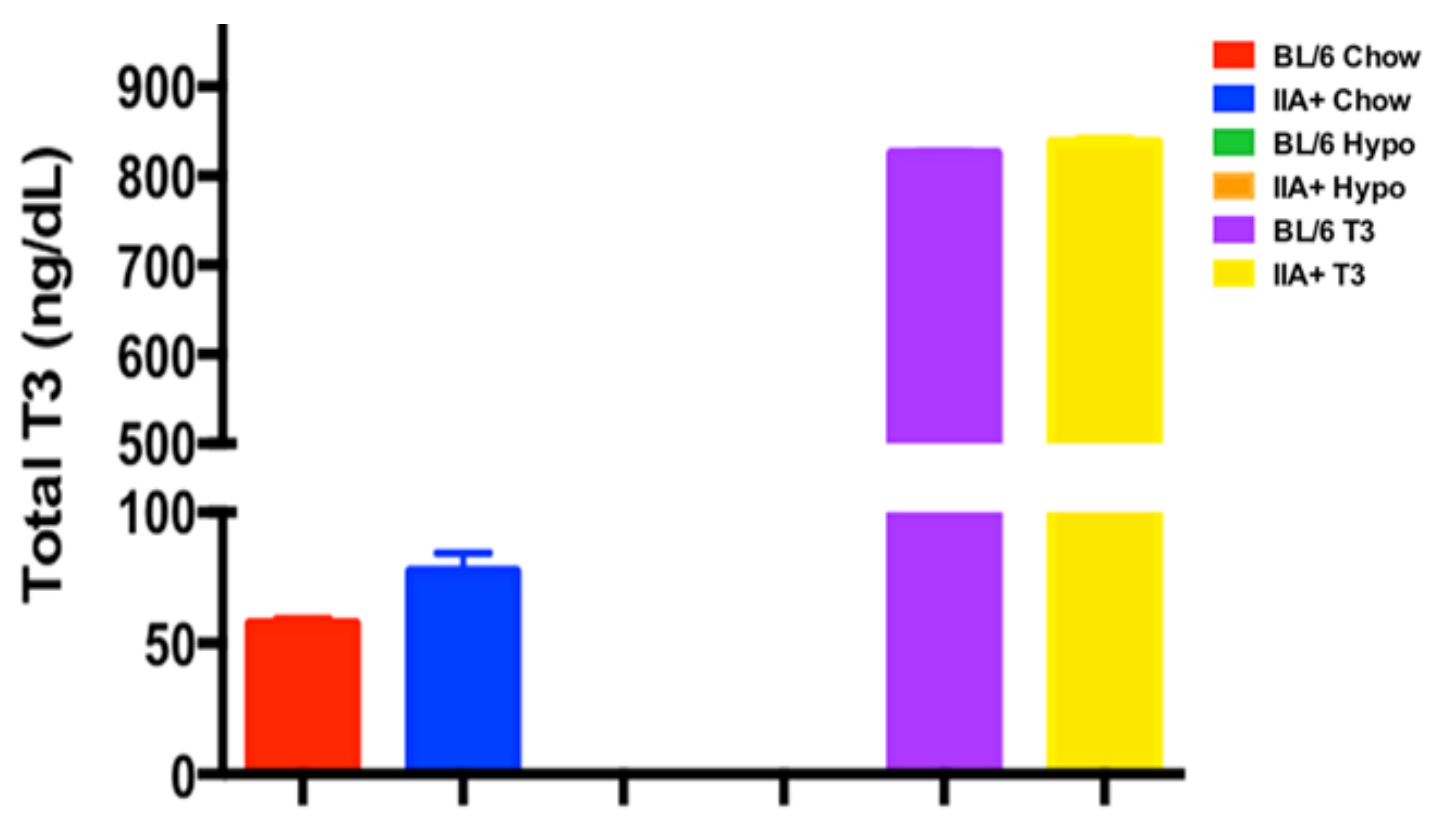

Figure 3-1. Serum T3 levels in mice on various thyroid diets

At the time of sacrifice, serum was collected from the mice and total thyroid hormone (T3) was measured at the University of Tennessee endocrinology laboratory. Data are expressed as total T3 in ng/dL serum. Each point represents the average T3 of 3 samples, which consisted of pooled (2-3 mice per sample) serum from the mice of their respective groups. 

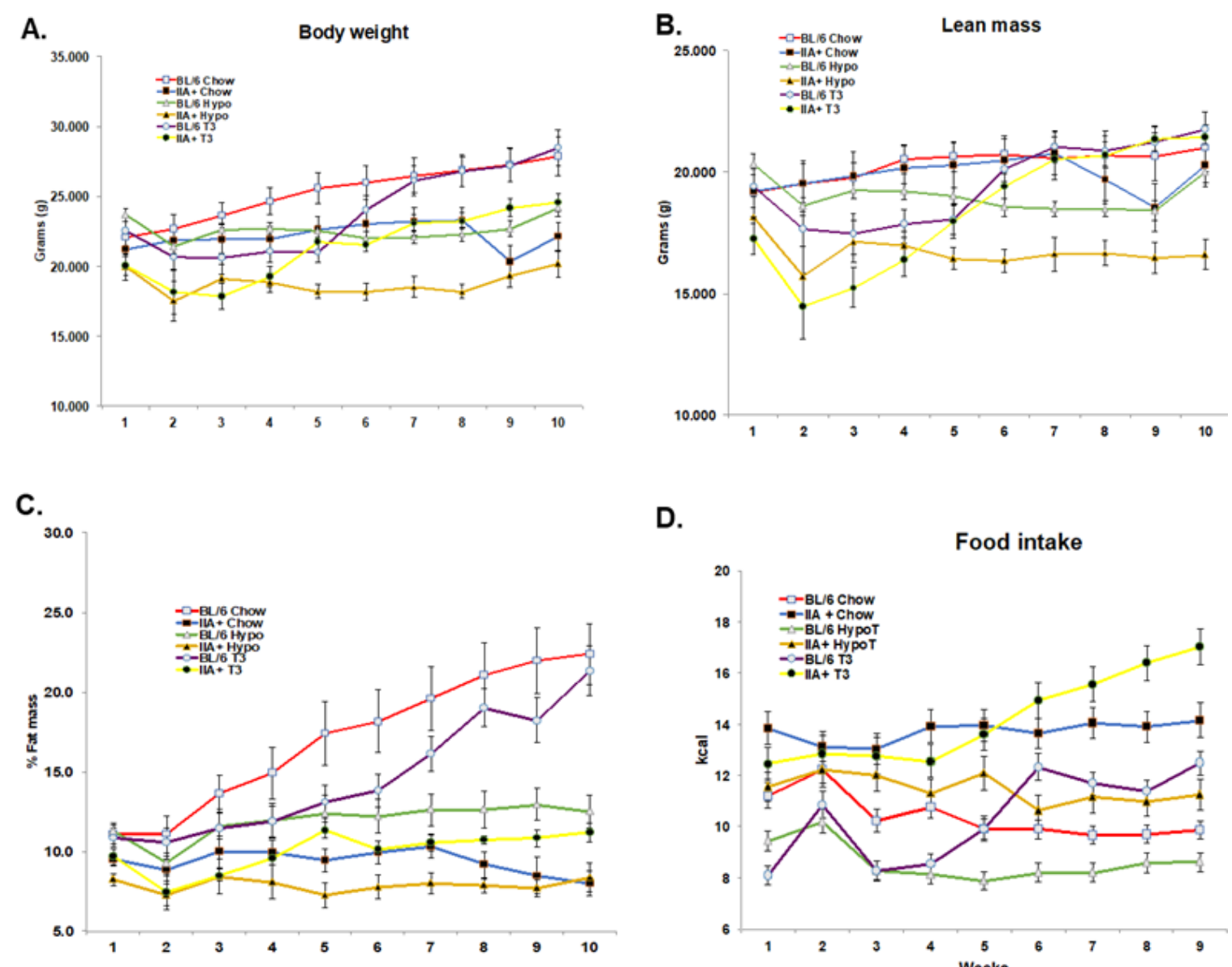

D.

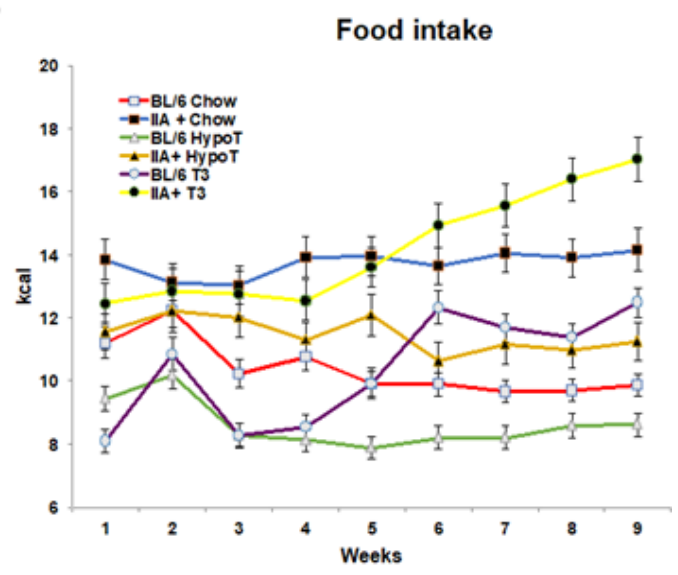

Figure 3-2. Body composition of IIA+ mice in response to thyroid diets

$\mathrm{C} 57 \mathrm{BL} / 6$ and IIA+ mice were placed on the various diets and treatments as described in the materials and methods. A. The weight gain (gms) of the mice in the 6 groups was assessed on a weekly basis. B. MRI analysis was used to determine the lean mass of the mice. C. The percent body fat was determined by MRI on a weekly basis. D. The average kcals consumed per cage were calculated from the food intake and the calorie content of the various diets. In all groups there were 7 to 11 mice. The differences in the various time points was determined by one-way ANOVA. 
$\mathrm{VO}_{2}$
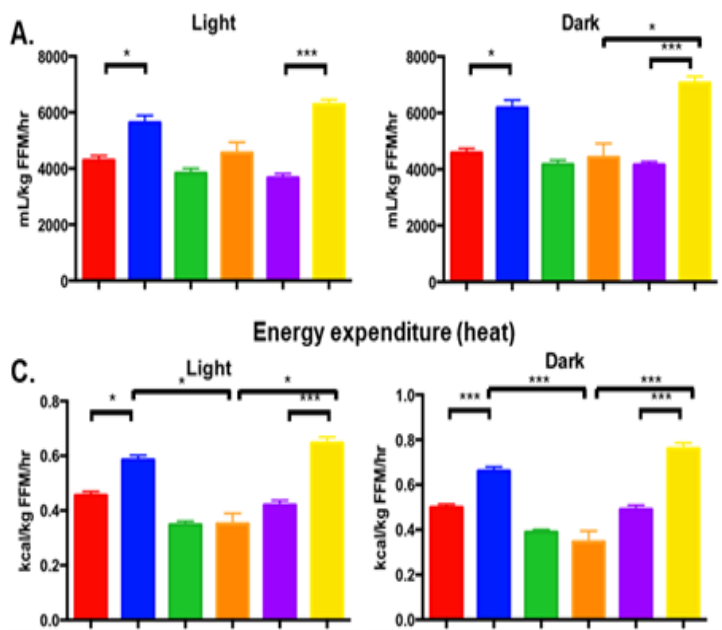

expenditure (heat)
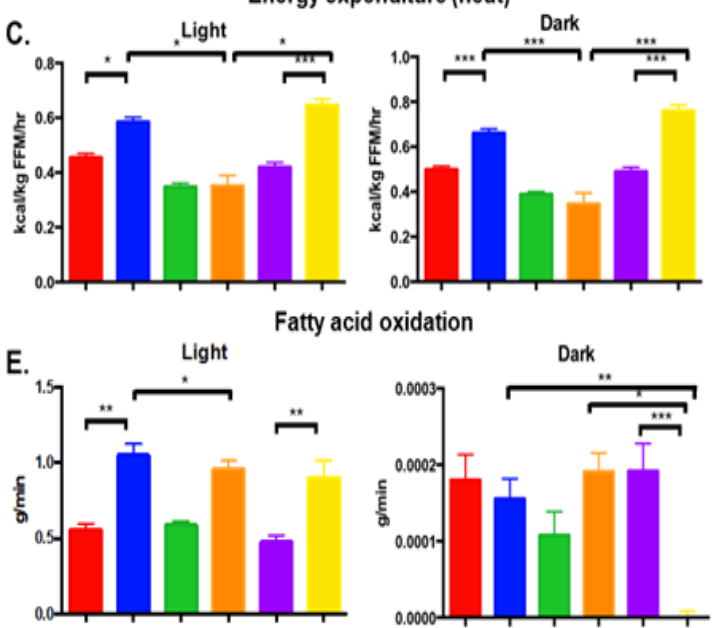

BL/6 Chow

- $11 \mathrm{~A}+$ Chow

BL/6 Hypo

IIIA $+\mathrm{Hypo}$

BL/6 T3

IIA $+\mathrm{T} 3$

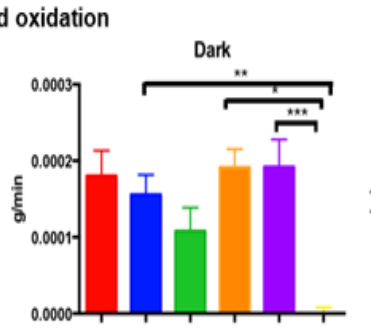

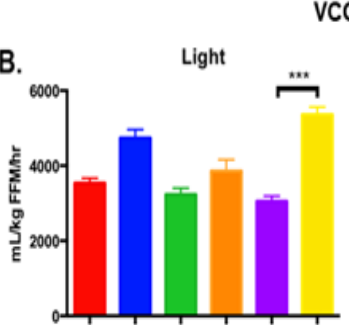

$\mathrm{CO}_{2}$

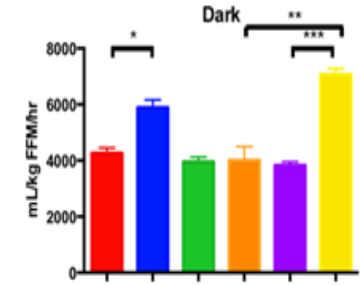

D.
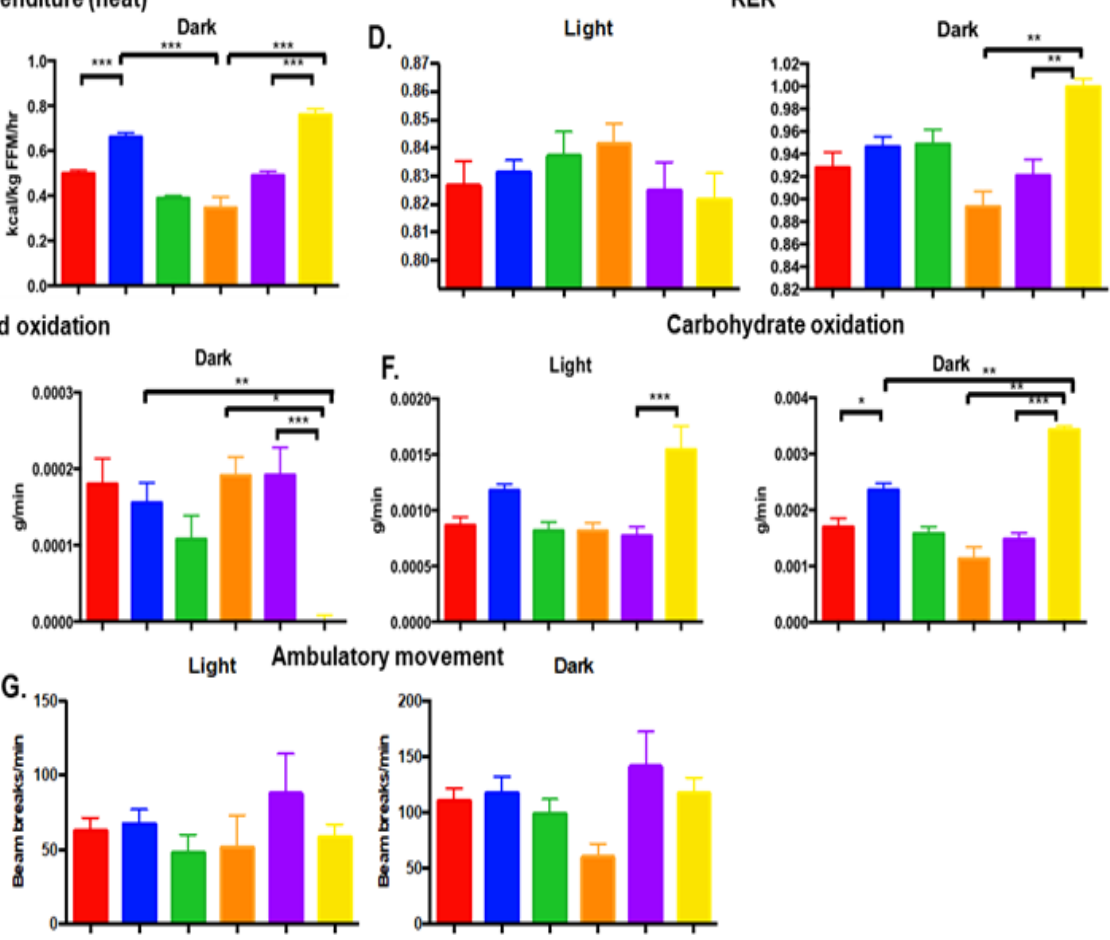

Figure 3-3. Metabolic cage analysis of mice on various thyroid diets

Metabolic analysis and activity of IIA + mice. After $10 \mathrm{wk}$ of exposure to the various diets and treatments, mice were placed individually in CLAMS chambers and their metabolism was assessed in both light and dark cycles. A, B) VO2 (A) and VCO2 (B) consumption were measured as millimeters per kilogram of lean mass per hour of $\mathrm{O} 2$ or $\mathrm{CO} 2$ consumption over a $24 \mathrm{~h}$ period. C) Energy expenditure (heat production) was expressed as kilocalorie/kilogram fat-free mass (FFM) per hour. D) RER was determined for all 6 groups of mice. E, F) Fatty acid (E) and carbohydrate oxidation (F) were expressed as grams per minute oxidized and calculated assuming negligible protein oxidation. G) Activity was determined by the number of beam breaks and is expressed as beam breaks per minute. Each assessment represents the averaged measurements of 7-11 animals over $7 \mathrm{~d}$ in the CLAMS chambers. The data was analyzed by ANCOVA, using lean mass as the covariate and each metabolic measurement as the dependent variable. ${ }^{*} \mathrm{P}$ $<0.05, * * \mathrm{P}<0.01, * * * \mathrm{P}<0.001$. 
oxidation drastically rose and fatty acid oxidation was minimal in the IIA + T3 mice (Figure 3-3E, F). Elevated carbohydrate oxidation was also observed in the IIA+ mice on chow (Figure 3-3E, F). While the IIA+ chow and IIA+ T3 mice had increased total energy expenditure, these changes did not appear to be in response to elevated ambulatory movement, as there were no significant alterations in physical activity in either the light or dark cycle. These data suggest that expression of PLA2G2A alters substrate preference in response to $\mathrm{T} 3$ from a mix of fatty acids and carbohydrates when at rest to almost entirely carbohydrates when the mice are active. These data also show that despite significant elevations in metabolic parameters in the IIA + mice, expression of PLA2G2A does not protect mice from reductions in energy expenditure in the hypothyroid state.

\section{Increased Insulin Sensitivity and Glucose Tolerance in IIA+ Mice}

Glucose and insulin tolerance were assessed following 7 or 8 weeks on the respective diets and/or treatments. The IIA+ mice on chow diet had much greater glucose tolerance compared to the BL/6 mice on chow (Figure 3-4A, B). Hypothyroidism abolished the elevated glucose tolerance in the IIA+ mice. Interestingly, the IIA + T3 treated mice had even greater glucose tolerance than the IIA+ chow mice, including lower fasting blood glucose. The IIA+ chow mice were more sensitive to insulin, as seen by the greater reduction in blood glucose following insulin injection and decreased total blood glucose over the 2 hour time period (Figure 3-4C, D). Most notably, the IIA+ T3 mice were extremely insulin sensitive. The IIA $+\mathrm{T} 3$ mice had reduced blood glucose $(<20 \mathrm{mg} / \mathrm{dL})$ following insulin administration to the point where several of the mice had to be administered glucose and excluded from the remainder of the test. These data indicate that expression of PLA2g2a enhances glucose tolerance and insulin sensitivity, and this effect is removed with hypothyroidism. We show that PLA2G2A expression may sensitize the mice to T3 and improve overall glucose homeostasis.

\section{Elevated UCP1 and GLUT4 in IIA+ BAT}

Interscapular BAT was harvested and the expression levels of several proteins that may be responsible for the elevations in metabolic parameters observed in the IIA+ mice were measured. The abundance of SIRT1 and PGC-1 $\alpha$, markers, commonly associated with mitochondrial biogenesis in BAT, were increased in IIA + mice relative to the BL/6 controls. Interestingly, the hypothyroid BL/6 mice had increased SIRT1 relative to the other BL/6 mice. (Figure 3-5A). UCP1 protein expression increased in the hypothyroid BL/6 mice compared to the BL/6 mice given T3. Additionally, UCP1 expression was induced in the IIA+ mice compared to their BL/6 counterparts in each case except the hypothyroid group (Figure 3-5C). EBF-2, a regulator of the brown adipocyte phenotype, was elevated in all the IIA+ mice compared to their BL/6 counterparts (Figure 3-5D). GLUT4, the primary glucose transporter in BAT, was markedly increased in the IIA + T3 mice compared to all other groups (Figure 3-5E). FATP1 was also significantly up-regulated in all IIA+ mice compared to their BL/6 
A.

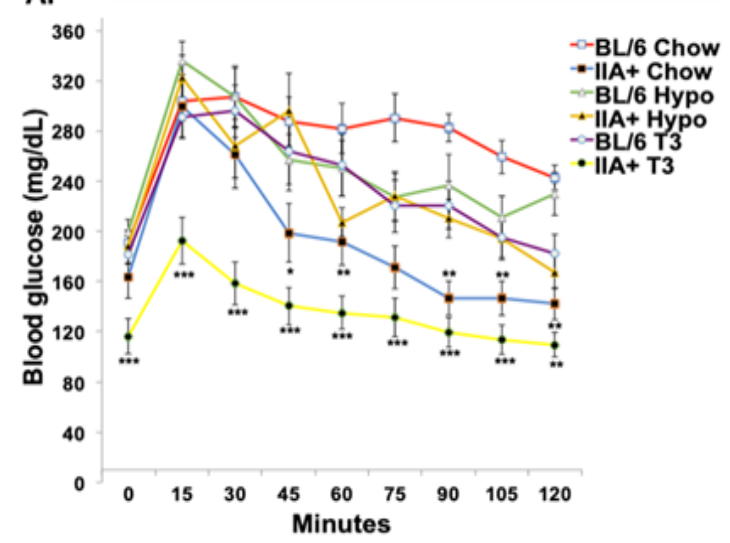

C. ${ }_{250}$

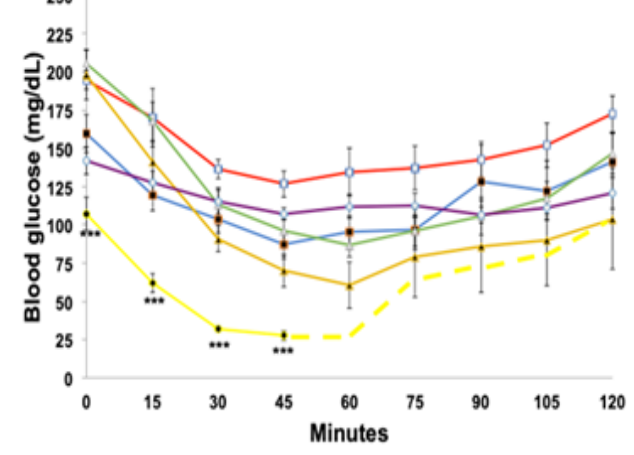

B.

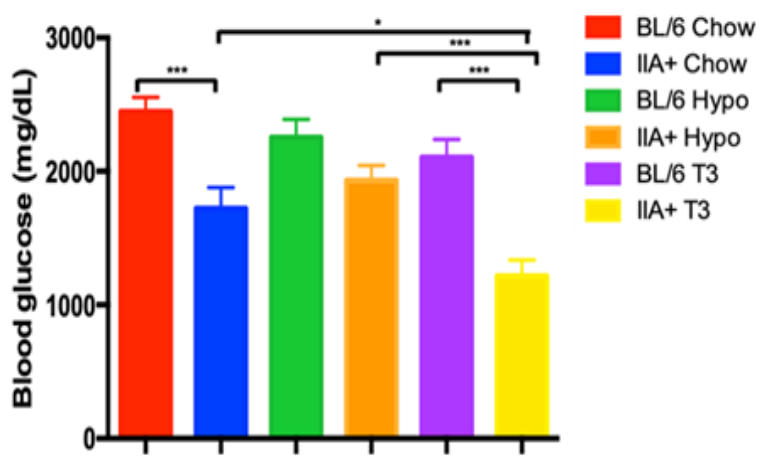

D.

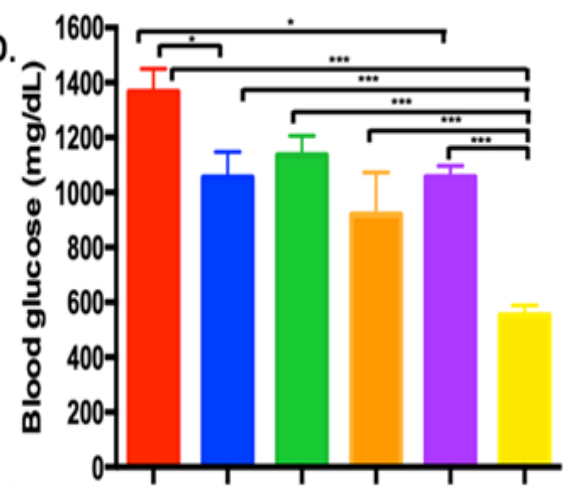

Figure 3-4. ITTs and GTTs of mice on various thyroid diets

A. Glucose tolerance tests (GTT) were conducted on all the mice in week 7 of the diet study as described in the materials and methods. The blood glucose was measured at 15 minute intervals. Each time point is the average of 7-11 mice. B. The total blood glucose (area under the curve) from all time points is shown from each group. C. Insulin tolerance tests (ITT) were conducted on all the mice in week 8 of the study as described in the materials and methods. The blood glucose was measured at 15 minute intervals following insulin injection. Each time point is the average of 7 to 11 mice. D. Total blood glucose for all time points in the ITT is shown. Differences in the glucose levels were determined one-way ANOVA. ${ }^{*} \mathrm{p}<0.05, * * \mathrm{p}<0.01, * * * \mathrm{p}<0.001$. 

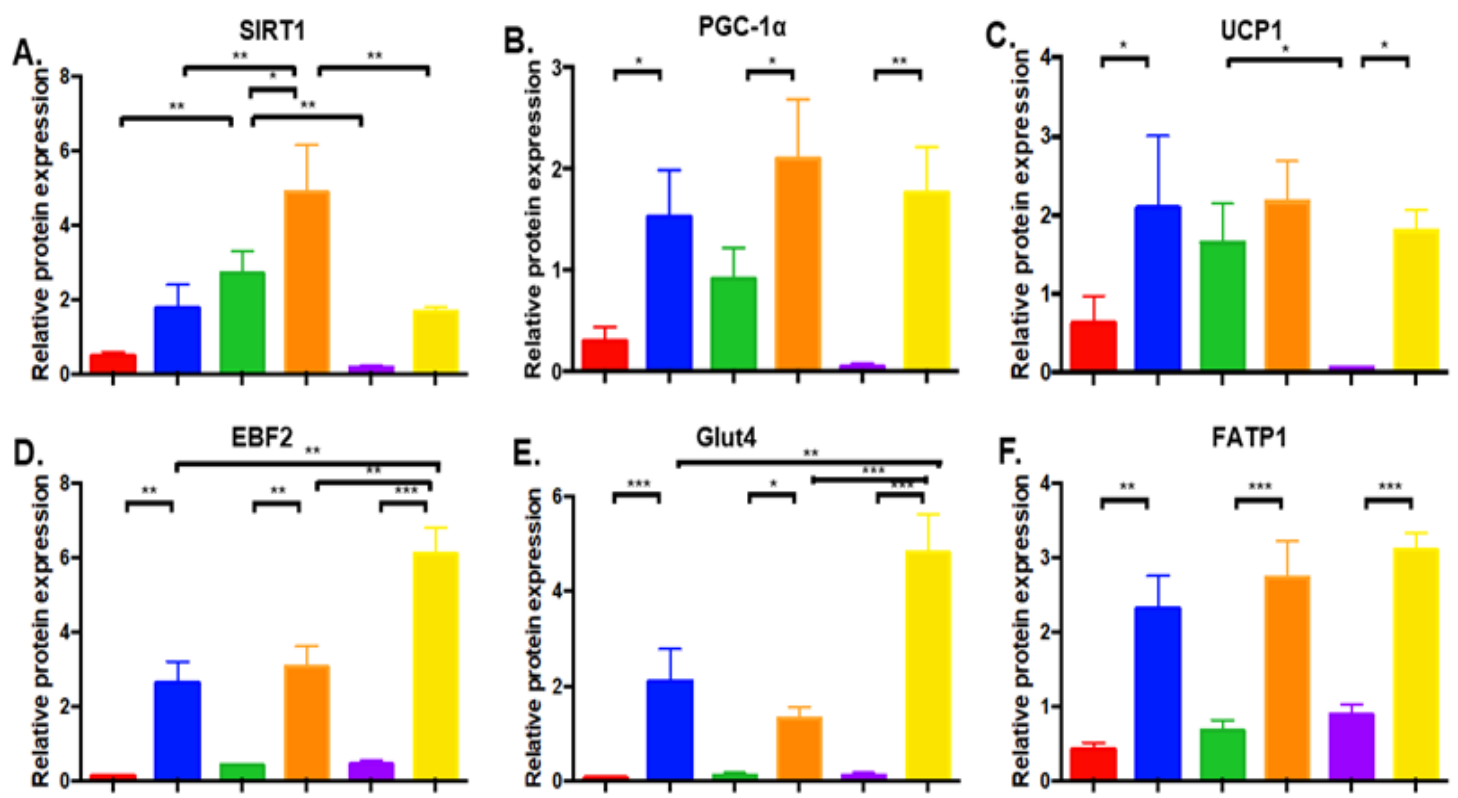

G.

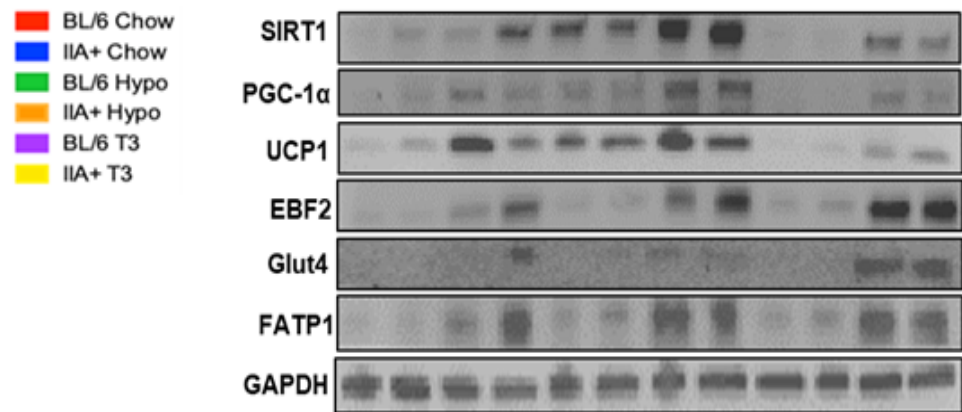

Figure 3-5. Thermogenic and substrate transport protein expression in BAT

Proteins were collected from the BAT at the end of the feeding period and the abundance of various proteins was analyzed by western blot. Representative westerns are shown and the data was quantified. A. SIRT1, B. PGC-1 $\alpha$, C. UCP1, D. EBF2, E. GLUT4, F. FATP1, G. Representative blots are shown. The data are expressed as relative protein abundance. Each point is the average protein expression of 4 animals. The data were analyzed by one-way ANOVA. ${ }^{*} \mathrm{p}<0.05, * * \mathrm{p}<0.01,{ }^{* * *} \mathrm{p}<0.001$. 
counterparts (Figure 3-5F). Finally, there were no differences in PLA2G2A protein levels between the IIA + mice in various thyroid states (data not shown). These data support enhanced total energy expenditure observed in the IIA+ mice. Increased FATP1 in the IIA + mice suggests an increase of fatty acid transport. Lastly, induction of GLUT4 in the $\mathrm{IIA}+\mathrm{T} 3$ treated mice suggests increased glucose transport into BAT.

\section{Thermogenic Protein Expression in IIA+ Adipocytes}

To further establish PLA2G2A's role in BAT uncoupling and WAT beiging, brown, epididymal (eWAT), and inguinal (iWAT) white adipocytes were obtained by differentiating pre-adipocytes harvested from the SVF of BL/6 and IIA+ mice (Figure 3-6A, B). In adipocytes from all adipose depots, the IIA + mouse adipocytes had elevated UCP1 abundance compared to the BL/6 adipocytes. This induction was much greater in the adipocytes from BAT and iWAT of the IIA+ mice, suggesting these depots are the primary regions of non-shivering adaptive thermogenesis and mitochondrial uncoupling. UCP1 abundance was also higher in the BAT of BL/6 adipocytes compared to the BL/ 6 adipocytes derived from eWAT depots. PGC- $1 \alpha$ and SIRT 1 expression were significantly enhanced in the IIA+ adipocytes from iWAT and BAT. BL/6 adipocytes did not contain PGC-1 $\alpha$ and there were no differences in SIRT1. PRDM16, one of the major transcription factors regulating beige/brown adipocyte thermogenesis (148), was elevated in the IIA+ adipocytes from all depots especially in BAT. PLA2G2A was not expressed in BL/ 6 adipocytes and was unchanged among adipose depots derived from the IIA+ mice. Brown pre-adipocyte differentiation rates were also unaffected by expression of PLA2G2A (Figure 3-7). Taken together, the induction of UCP1 and other markers that determine beige/brown adipocyte cell fate suggests that expression of PLA2G2A in mice enhances adaptive thermogenesis in adipose tissue.

\section{Hepatic Protein Expression in IIA+ Mice}

Differences in fuel utilization and fat accumulation, as well as changes in insulin sensitivity prompted us to examine protein expression in the liver. Both total and phosphorylated p70S6 kinase (Thr389) (p70S6K) were induced in IIA+ mice treated with T3, suggesting enhanced insulin signaling (Figure 3-8A, B). Given the increase in fatty acid oxidation in the IIA+ mice, we examined FATP1 in the liver as well. FATP1 expression was induced in the IIA+ mice, and this effect was abolished when the mice were made hypothyroid (Figure 3-8C). The precursor SREBP-1c was elevated in the IIA + mice, but this effect was abolished when the mice were made hypothyroid (Figure 3-8E). Nuclear SREBP-1c levels were also enhanced in the IIA+ mice, although to a lesser degree than precursor (Figure 3-8D). Since the IIA+ mice had elevated UCP1 in BAT, increased food intake, and increased $\mathrm{VO} 2$, we assessed liver expression of uncoupling protein 2 (UCP2). UCP2 was markedly elevated in the IIA + T3 treated mice compared to all other groups (Figure 3-8F). Finally, SIRT1 abundance was enriched in both hypothyroid groups (Figure 3-8H). 


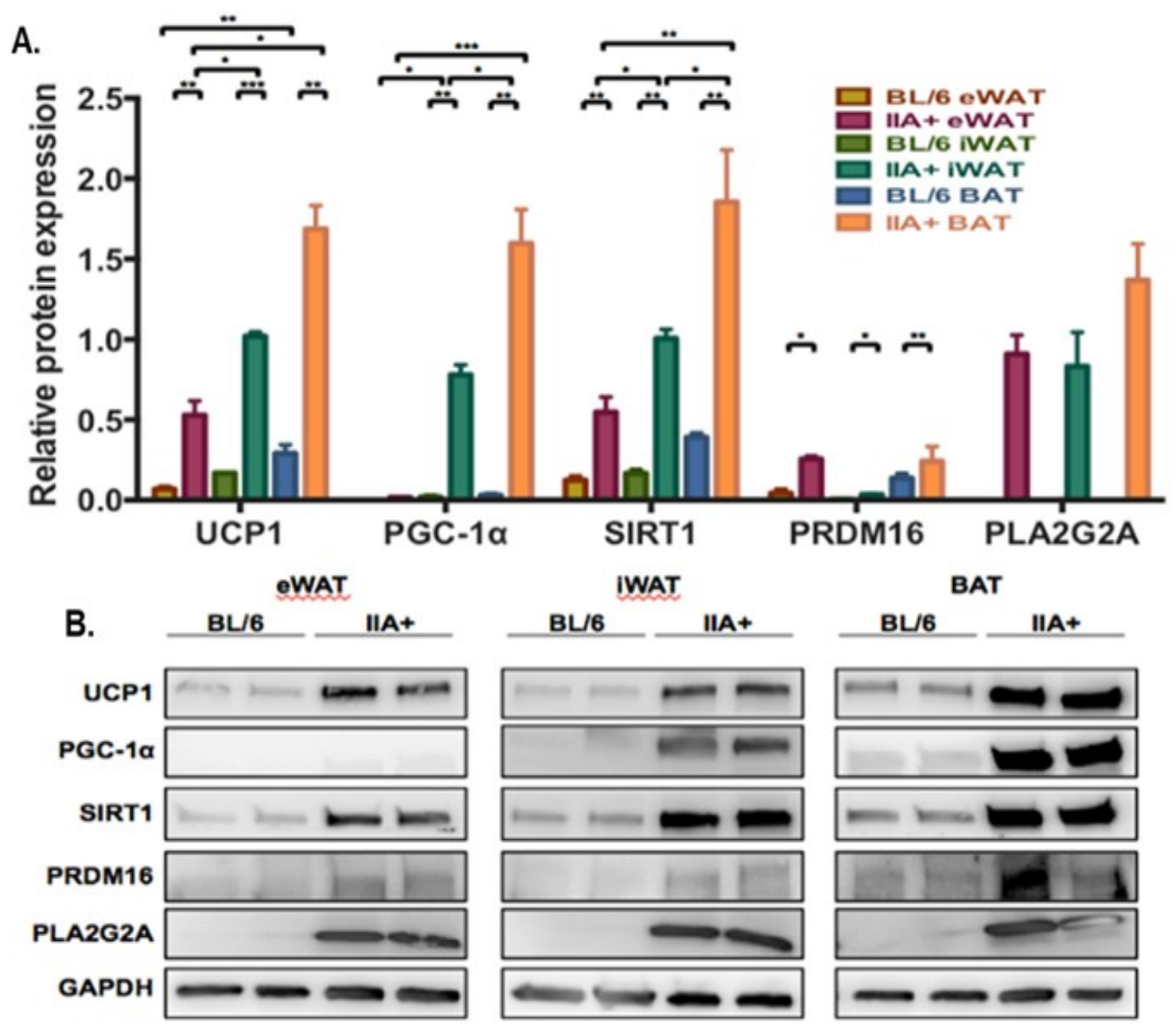

Figure 3-6. Thermogenic protein expression elevated in IIA+ adipocytes

Mitochondrial uncoupling and increased beiging in adipocytes of IIA + mice. Adipocytes from eWAT, iWAT, and BAT were derived from the SVF of BL/ 6 and IIA+ mice. Following differentiation, protein was harvested from adipocytes and factors involved in WAT beiging and mitochondrial uncoupling were examined. A) Relative protein expression of metabolic proteins. B) Representative Western blots. The data are expressed as protein abundance relative to GAPDH. Each point is the average protein expression of 4-5 $10 \mathrm{~cm}$ cell culture plates of adipocytes, which contained adipocytes pooled from 3 to 5 animals \pm se. The data were analyzed by 1 -way ANOVA. $* \mathrm{P}<0.05$, $* * \mathrm{P}<0.01, * * * \mathrm{P}<0.001$. 

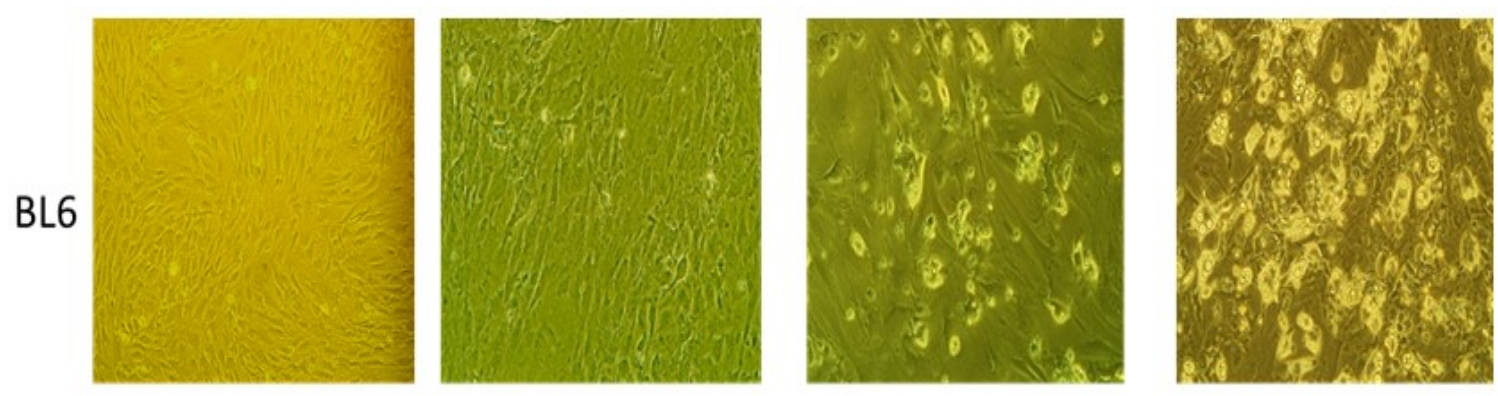

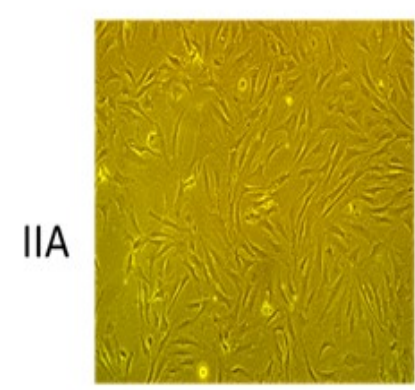

Day 0

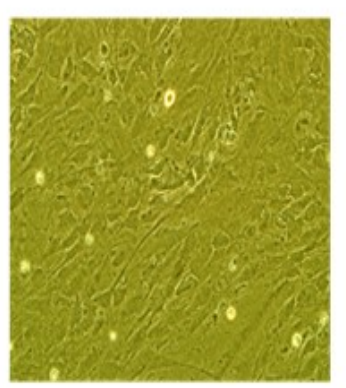

Day 2

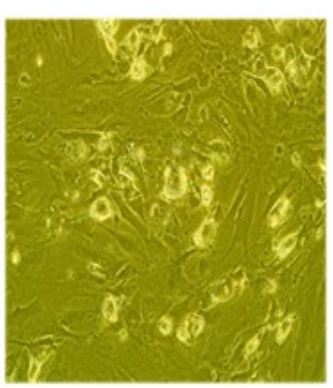

Day 4

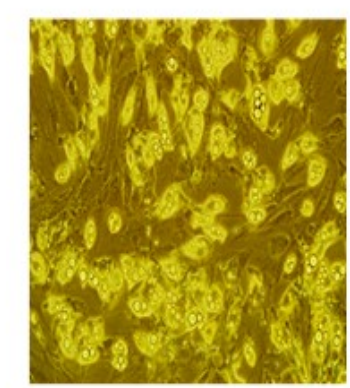

Day 7

Figure 3-7. Differentiation timeline of C57BL/6 and IIA+ adipocytes

SVF was seeded in 6-well plates and induced to differentiate into mature adipocytes over 7 days according to the protocol described in methods. Cells were imaged by inverse microscopy and no differences in differentiation rates were found. 


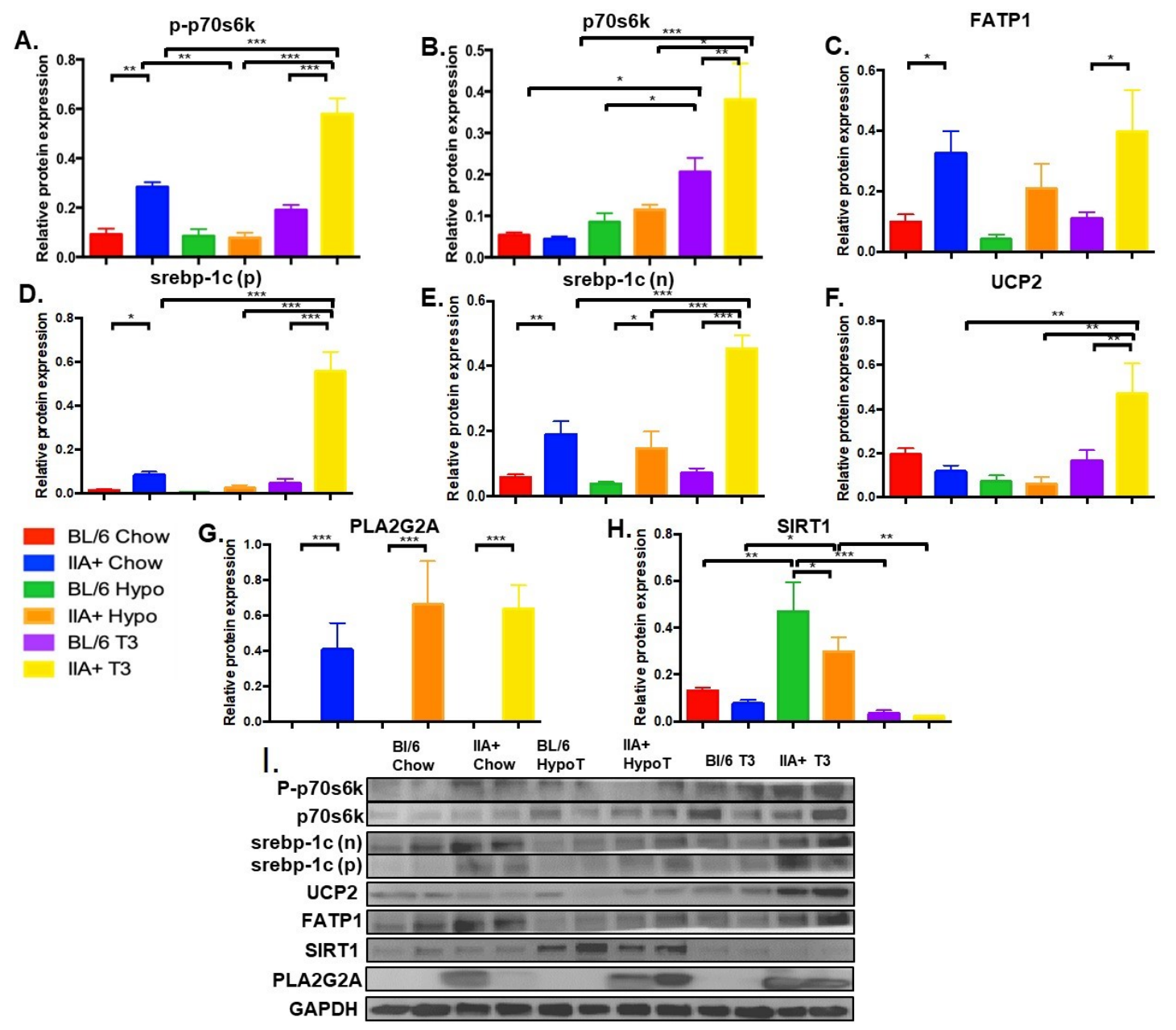

Figure 3-8. Hepatic protein expression in mice on various thyroid diets

Proteins were collected from the livers at the end of the thyroid treatments, the abundance of various proteins was analyzed by Western blot, and the data was quantified. A-H) Relative protein expression of phosphorylated p70S6K (Thr389) (A), total p70S6K (B), FATP1 (C), nuclear SREBP-1c (D), precursor SREBP-1c (E), UCP2 (F), PLA2G2A (G), SIRT1 (H). I) Representative Western blots. The data are expressed as protein abundance relative to GAPDH. Each point is the average protein expression of 4-5 animals \pm se. The data were analyzed by 1 -way ANOVA. $* \mathrm{P}<0.05, * * \mathrm{P}<0.01$, $* * * \mathrm{P}<0.001$. 


\section{Discussion}

Here, we show that PLA2G2A enhances BAT uncoupling and the subsequent browning of WAT through the induction of multiple factors involved in the determination of beige/brown adipocyte identity. Furthermore, proteins related to fatty acid and glucose transport in BAT were markedly enhanced, likely contributing to the increased total energy expenditure seen in the IIA+ mice. Surprisingly, we also observed that thyroid hormone administration enhances the insulin sensitizing effects of PLA2G2A resulting in a drastic increase in insulin signaling and glucose tolerance. Lastly, T3 treated IIA+ mice switched their substrate utilization to almost exclusively carbohydrates when they were active. My data show that PLA2G2A plays an important role in the regulation of several important metabolic processes.

As stated in Chapter 1, studies have been conducted on the role of sPLA2s in metabolism and the associated pathologies such as obesity, diabetes and dyslipidemia. Currently, at least six the eleven sPLA2 family members have been shown to contribute to metabolic regulation (143). PLA2g5, which catalyzes the hydrolysis of phospholipids in lipoproteins, is upregulated in white adipose tissue of obese mice (69). Additionally, Pla2g5-/- mice had significantly elevated plasma LDL, increased obesity and showed lower insulin sensitivity when fed a high-fat diet, , suggesting that PLA2g5 has an antiobesogenic action (69). PLA2g1b is highly expressed in the intestine and is involved in dietary lipid absorption. Pla2g $1 b$-/- mice were resistant to obesity and had improved insulin signaling and glucose tolerance when fed both a high fat and high carbohydrate diet (128). PLA2g2e is another sPLA2 expressed in only a few tissues including epididymal adipose (56). The metabolic role of PLA2g2e is controversial. One group using Pla2g $2 e-/-$ mice found that this sPLA2 promoted lipid accumulation in adipose tissue and liver (69). In contrast, a more recent study found that C57BL/6 mice deficient in Pla2g2e had elevated lipid accumulation and perturbations in lipolysis despite no difference in food intake (73). This second study proposed a protective role for PLA2g2e with respect to weight gain. Importantly, murine PLA2g2e has been proposed to be homologous to PLA2G2A, suggesting that PLA2g2e possesses a similar role in mice that PLA2G2A has in humans (149). Here, we have shown that similar to PLA2G2E, PLA2G2A overexpression protects mice from weight gain and lipid accumulation, despite increases in food intake.

Given the enhanced metabolic phenotype observed in the IIA + mice, we hypothesized that expression of PLA2G2A would counteract the metabolic effects typically seen in hypothyroidism, such as reductions in energy expenditure $(150,151)$. However, we show that PLA2G2A expression cannot counterbalance the lack of T3, as energy expenditure was still reduced in the hypothyroid IIA+ mice (Figure 3-2). An unexpected finding was that the IIA+ mice treated with T3 altered their fuel substrate utilization to almost completely carbohydrates during their active cycle as indicated by an RER of approximately 1.0 (Figure 3-2D). Fatty acid and carbohydrate oxidation rates were calculated according to the non-protein respiratory quotient described previously (145), and replicate the extremely high carbohydrate utilization of the IIA+ mice given $\mathrm{T} 3$ in the active phase (Figure 3-2E, F). It is well known that cold exposure activates 
BAT adaptive thermogenesis through induction of a thermogenic set of genes including PGC-1 $\alpha$ and UCP1, resulting in increased BAT recruitment and energy expenditure (152154). Previous studies found this cold-induced activation of BAT significantly enhanced RER, indicating elevated glucose utilization (155). Furthermore, T3 administration increases GLUT4 expression, the primary insulin-regulated glucose transporter in BAT (156) and in differentiating brown adipocytes from rats (157). Similar results were also found in human subjects where hyperthyroid patients had as much as a 3-fold increase in glucose uptake in BAT compared to euthyroid patients (158). We observed similar changes in the IIA+ mice treated with T3 as all of the IIA+ mice had elevated GLUT4 protein expression and T3 augmented this effect (Figure 3-4E, G). The increased carbohydrate utilization (Figure 3-2D) in the IIA+ mice treated with T3 likely stems in part from T3-mediated increases in GLUT4 in BAT, which would increase glucose transport and utilization by the BAT itself.

We also found that the IIA+ mice treated with T3 were extremely insulin sensitive. We had previously reported greater insulin sensitivity in the IIA+ mice (51). In part, the enhanced insulin sensitivity might arise from the lean phenotype of the mice, but the administration of thyroid hormone further enhanced the glucose tolerance and insulin responsiveness (Figure 3-3). The mice had much lower fasting glucose and very high glucose utilization in active phase of the circadian cycle (Figure 3-2D, F). The increased GLUT4 expression in BAT (Figure 3-4E) and proteins in the liver involved in insulin signaling, such as p-p70S6K (Thr389) and nuclear srebp-1c (Figure 3-6A, D), supports the finding of high insulin sensitivity in these mice.

Previously, we reported that IIA+ mice had elevated mitochondrial uncoupling in BAT (51). We postulated that a mechanism for this increase was an elevation in circulating lysophospholipids and fatty acids caused by PLA2G2A glycerophospholipid hydrolysis to fuel BAT and to activate UCP1, thus increasing energy expenditure and overall metabolic rate. Here, we further characterized the enhanced metabolic phenotype of the IIA + mice by showing that positive regulators of UCP1 expression, such as SIRT1 and PGC-1 $\alpha(120,121)$, as well as UCP1 abundance itself are induced in the IIA+ mice (Figure 3-4A, B, and G). These metabolic regulators were also increased in the mice on the hypothyroid diet, which is likely a compensation mechanism in order to counteract the reductions in VO2 and total energy expenditure. The development, determination, and maintenance of brown adipose tissue is controlled by a particular set of transcription factors and genes (159). One of these transcription factors is EBF2 which cooperates with peroxisome proliferator-activated receptor gamma (PPAR $\gamma$ ) to positively influence brown adipocyte cell fate and enhance BAT development (160). Regardless of hypothyroid or euthyroid state, the IIA+ mice had significantly elevated EBF2 protein expression in BAT, and this elevation was further increased by T3 treatment (Figure 3-4D, G). The IIA + mice also showed elevated expression of one of the major fatty acid transporters in adipose tissue (Figure 3-4F), fatty acid transport protein 1 (FATP1), which increases long chain fatty acid uptake and is required for nonshivering adaptive thermogenesis in BAT $(161,162)$. 
WAT beiging, or the transformation of WAT from an energy-storing tissue to an energy-dissipating tissue, has gained considerable interest in recent years due to its potential as a therapeutic in the treatment of metabolic diseases including obesity, hyperlipidemia and cardiovascular disease. This beiging entails increased expression of BAT-specific proteins in WAT to regulate thermogenesis (163). We examined whether or not adipocytes derived from various adipose depots in IIA + mice showed characteristics of WAT beiging. Adipocytes from iWAT and eWAT displayed increased abundance of many of the major factors involved in beiging, including UCP1, SIRT1, and PRDM16 (Figure 3-5A, B). Importantly, we did observe an induction of PGC-1 $\alpha$ in iWAT, but not eWAT in the IIA+ adipocytes, suggesting that iWAT has greater potential for beiging than eWAT. Previous studies by other labs have found that iWAT readily accumulates beige-like cells in response to cold exposure and this effect is seldom observed in eWAT (164). Although my observations do not entirely concur with these findings, the IIA+ adipocytes from iWAT do have increased abundance of brown adipogenic proteins compared to those from eWAT (Figure 3-5A, B). Brown adipocytes were also derived from the BAT of BL/ 6 and IIA+ mice and brown adipogenic proteins were significantly higher in the IIA+ adipocytes, indicating that PLA2G2A not only potentiates beiging of WAT, but activates uncoupling in BAT as well.

Uncoupling protein 2 (UCP2) is another family member of the uncoupling proteins expressed in tissues such as skeletal muscle, heart, kidney, and liver, and it is hypothesized to have a unique role in energy balance and body weight regulation (165). UCP2 mRNA is induced in rats administered T3, and this upregulation may play a role in the elevation of energy expenditure caused by T3 treatment (166). Other studies have found that hepatic UCP2 expression is enhanced in response to obesity in $o b / o b$ mice (167). Here, we observed significantly upregulated hepatic UCP2 abundance in the IIA+ mice administered T3 compared to all other groups (Figure 3-8F). While these mice were very lean and displayed little, if any obesogenic characteristics, their daily energy intake exceeded all other mice, which may have an impact on UCP2 regulation according to studies mentioned earlier on the $o b / o b$ mice (167).

One surprising observation was the increased expression of hepatic SIRT1 in the hypothyroid mice (168). Earlier, we stated that many of the beige/BAT markers for adaptive thermogenesis were induced in the hypothyroid mice as a compensation mechanism to counteract their low energy expenditure and metabolism (Figure 3-5). These hypothyroid mice ate much less food as well (Figure 3-2D). We hypothesize that an elevation in hepatic fatty acid oxidation may be compensatory to their reduction in food intake.

Finally, previous studies from the Park lab showed that thyroid hormone inhibited the expression of the rat, mouse, and human PLA2g2a gene. However, this previous work involved acute administration of T3 either to hypothyroid mice or cells. In these studies, we did see an increase in PLA2G2A in the hypothyroid mouse livers, but it did not reach statistical significance (Figure 3-8G, I). PLA2G2A was also not repressed by T3 administration. However, we used one tenth the T3 dose so as to restore the mice to euthyroidism over a 5 week period, rather than acute administration. We speculate that 
the difference in these studies from the previous work arises from the dose and the length of the $\mathrm{T} 3$ treatment.

These data show that introduction of human PLA2G2A in mice enhances total energy expenditure through upregulation of a $\mathrm{BAT} /$ beige thermogenic program in brown and white adipocytes to combat fat accumulation. We also show that PLA2G2A and thyroid hormone work synergistically to drastically improve insulin sensitivity and glucose tolerance. Finally, we found that although PLA2G2A improves total energy expenditure, it cannot protect mice from reductions in energy expenditure associated with hypothyroidism. We have identified and further characterized PLA2G2As role in metabolism in response to variations in thyroid status. Future studies will determine mechanisms behind the role of PLA2G2A in BAT/beige thermogenesis. 


\section{CHAPTER 4. PLA2G2A AUGMENTS ADIPOSE TISSUE THERMOGENESIS}

\section{Introduction}

Brown adipose tissue (BAT) is the primary site of adaptive thermogenesis in mammals $(153,169)$, and increased activity of BAT can protect from obesity and symptoms of type 2 diabetes such as hyperglycemia and insulin resistance $(170,171)$. High BAT activity increases the transport and oxidation of metabolites such as glucose and fatty acids. The energy from these metabolites is then dissipated as heat by the uncoupling of the electron transport chain from oxidative phosphorylation, a process that is mediated by a mitochondrial uncoupling protein - uncoupling protein 1 (UCP1) (172, 173). In addition, it has been discovered that BAT acts as a secretory organ similar to white adipose tissue (WAT), secreting factors including lipids and microRNAs known as 'brown adipokines.' These act in an autocrine-paracrine fashion and are pivotal to BAT thermogenic function (174-176). This knowledge has provoked new research avenues for BAT as a therapeutic target to combat obesity and metabolic disorders.

As mentioned in the first 3 chapters of this dissertation, many of the sPLA2s play significant roles in the protection from obesity and metabolic syndrome through various mechanisms related to lipid transport and metabolism. In Chapter 2, we described the phenotype of the C57BL/6 PLA2G2A over-expressing mice (IIA+ mice) in response to a high-fat diet for 10 weeks. The IIA+ mice were resistant to diet-induced obesity and remained extremely insulin sensitive compared to their BL/6 counterparts (51). IAs outlined in Chapter 3, further assessment of the IIA+ animals revealed that they had increased thermogenesis based on metabolic cage analysis and significantly active BAT, including enhanced expression of BAT-specific thermogenic markers such as UCP1, PGC-1 $\alpha$, sirtuin-1 (SIRT1) and PRDM16 (52).

Here, we investigated how PLA2G2A improves adipose tissue-specific thermogenesis in the mice and in brown adipocytes. We found that IIA+ mice displayed elevated oxygen consumption and energy expenditure during thermoneutral conditions. Administration of recombinant PLA2G2A protein to brown adipocytes from BL/6 animals resulted in significant elevation of UCP1 and PRDM16. Microarray analysis of BAT revealed elevated expression of numerous genes related to fuel transport and oxidation. These data have further characterized the novel role of PLA2G2A in obesity and metabolism.

\section{Materials and Methods}

\section{PLA2G2A Expressing C57BL/6 Mice (IIA+)}

The IIA + mice were provided by Dr. Eric Boilard at the Universite Laval (CHUL), Quebec, Canada (51). Male mice heterozygous for the PLA2G2A gene were 
bred with C57BL/6 (BL/6) females. For these experiments, male BL/6 and IIA+ mice were used. Mice were housed according to genotype and fed standard chow Teklad LM485 (7012) diet, which contained 5\% of the calories as fat. Two or three littermates per cage of the same genotype and diet were housed with a constant light and dark phase of 12 hours at $20-23^{\circ} \mathrm{C}$. All animal procedures were approved by the UTHSC Institutional Animal Care and Use Committee.

\section{Metabolic Cages}

Mice were individually housed in the comprehensive lab animal monitoring system (CLAMS) chambers and maintained on the chow diet for 1 week. During the initial 4 days, mice were housed at an ambient room temperature of approximately $22^{\circ} \mathrm{C}$. For the final 3 days the temperature was increased to approximately $28^{\circ} \mathrm{C}$. Total energy expenditure was determined using indirect calorimetry. VO2 and heat production are expressed relative to fat-free mass. The respiratory exchange ratio (RER) was measured to determine energy substrate preference. Physical activity was determined by the number of infrared beam breaks.

\section{Primary Culture of Adipocytes}

The stromal vascular fraction (SVF) was collected from the subcutaneous white and brown (interscapular) adipose tissue of 4-6 week old BL/6 and IIA+ mice using an adipocyte isolation and differentiation protocol described previously (146). Tissue was collected and digested in a solution containing collagenase, phosphate-buffered saline (PBS), and calcium chloride $\left(\mathrm{CaCl}_{2}\right)$, an activator of collagenase. After digestion, the tissue suspension containing the SVF was filtered through a $100 \mathrm{uM}$ cell strainer, pelleted in a bench top centrifuge, washed twice with PBS, and plated in collagen-coated dishes. Cells were maintained in Dulbecco's modified eagle's medium nutrient mixture F-12 Ham (DMEM/F12) containing 10\% fetal bovine serum (FBS) and an antibiotic solution containing penicillin and streptomycin $(\mathrm{P} / \mathrm{S})$. After the SVF cells reached complete confluence, the SVF cells, which contained pre-adipocytes, were switched to an induction cocktail for adipocyte differentiation. The induction cocktail was composed of rosiglitazone, dexamethasone, 3-isobutyl-1-methylxanthine (IBMX) and insulin (147). After 2 days of induction, the cells were incubated in the maintenance media containing DMEM/F12, 10\% FBS, $1 \mathrm{uM}$ insulin, $1 \mathrm{nM} \mathrm{T3}$, and $1 \mathrm{uM}$ rosiglitazone. The cells were maintained in this media for 7 days with the media being replaced every other day. Adipocytes were prepared from pooled adipose samples from 5 animals.

\section{Seahorse Mitochondrial Stress Test}

Seahorse experiments were conducted in collaboration with Dr. Heather

Smallwood in the Department of Pediatrics at the University of Tennessee Health Science Center using the Xfe96 bioanalyzer. Each adipocyte preparation was derived from pooled 
WAT or BAT from 5 animals. The adipocytes were differentiated in $10 \mathrm{~cm}$ dishes and then transferred to 96 well plates designated for the seahorse Xfe96 bioanalyzer at 20,000 cells/well for analysis. There were 12 replicate wells for each preparation of adipocytes. Oligomycin, FCCP (carbonilcyanide p-triflouromethoxyphenylhydrazone, $2 \mu \mathrm{M}$ ), and Rotenone/Antimycin A were added to the cells at 15, 35, and 55 minutes.

\section{Western Blots}

Total protein from BAT and subcutaneous WAT was isolated in RIPA buffer containing protease inhibitors [50 mM Tris- $\mathrm{HCl}$ (pH 7.4), $100 \mathrm{mM} \mathrm{NaCl}, 5 \mathrm{mM}$ EDTA (pH 8.0), 1\% Triton, $1 \mathrm{mM}$ benzamidine, and $0.5 \mathrm{mM}$ PMSF]. Protein lysates were prepared in loading buffer and equal amounts were loaded on 4-20\% gradient Tris/glycine precast acrylamide gels. Proteins were separated using SDS-PAGE and transferred to nitrocellulose membranes. Membranes were blocked with 5\% nonfat milk in TBS-T prior to antibody incubation. Membranes were immunoblotted with the appropriate primary antibody in 5\% BSA in TBS-T and secondary antibody in 5\% nonfat milk in TBS-T. Immunoreactive proteins were detected using Super Signal West Femto chemiluminescent substrate (Thermo Scientific) (46). The following antibodies were used: $\beta$-actin, catalog number 13E5 from Cell Signaling Technology; uncoupling protein 1 (UCP1), catalog number D9D6X from Cell Signaling Technology; GAPDH, catalog number 14C10 from Cell Signaling Technology; PLA2G2A, catalog number AB23705 from AbCam; sirtuin 1 (SIRT1), catalog number A21993 from Life Technologies; PRDM16, catalog number Ab106410 from AbCam; PGC-1 $\alpha$, catalog number NBP104676SS from Novus Biologicals; pAMPK (Thr172), catalog number 40H9 from Cell Signaling Technology; AMPK, catalog number D5A2 from Cell Signaling Technology; Total Oxphos rodent antibody cocktail, catalog number ab110413 from AbCam.

\section{Citrate Synthase Activity}

BAT, subcutaneous WAT, and quadriceps muscle were harvested from 8 C57BL/6 and 8 IIA+ male mice. Citrate synthase activity was measured by detecting the change in absorbance per minute of 5,5' - dithiobis-2-nitrobenzoate (DTNB) on a plate reader at $412 \mathrm{~nm}$ following the addition of oxaloacetate to tissue homogenized in a homogenization buffer $(0.175 \mathrm{M} \mathrm{KCL}, 2.0 \mathrm{mM}$ EDTA in H20, $\mathrm{pH}$ 7.4). The results are expressed as the average change in absorbance per minute per gram of adipose tissue.

\section{Recombinant PLA2G2A Enzyme}

Recombinant PLA2G2A enzyme was provided by Dr. Gerard Lambeau at the Université Côte d'Azur, Centre National de la Recherche Scientifique. PLA2G2A enzyme was added to mature brown adipocytes harvested from C57BL/6 animals at a concentration of $200 \mathrm{ng} / \mathrm{mL}$ for 24 hours. After 24 hours, cells were harvested and the levels of PRDM16, UCP1 and GAPDH were observed. 


\section{Adipocyte Immunohistochemistry}

Brown adipocytes were prepared from BAT of BL/6 and IIA+ mice. SVF cells were seeded on coverslips in 6-well plates and maintained in DMEM/F12 media containing 10\% FBS. Images were taken after 7 days of incubation with differentiation media and are presented at 20x magnification. Representative images of brown adipocytes stained with DAPI, UCP1, and MitoTracker are shown. Cells on coverslips were incubated with primary antibodies for 1 hour (MitoTracker Red CMXRos, catalog number 9082 from Cell Signaling Technology; UCP1, catalog number Ab10983 from AbCam). The antibodies were then incubated in secondary antibody for 30 minutes at room temperature using anti-rabbit IgG Fab2 Alexa Fluor 594 molecular probe (Cell Signaling Technology, 8889S) for MitoTracker staining (red) and anti-mouse IgG Fab2 Alexa-Fluor 488 molecular probe (Cell Signaling Technology, 4408S) for UCP1 staining (green).

\section{Affymetrix RNA Microarray}

BAT was harvested from 5 C57BL/6 and 5 IIA+ animals and stored in liquid nitrogen. Total RNA was extracted from BAT using the RNeasy Lipid Tissue Mini Kit (QIAGEN, catalog number 74804) and treated with DNase I (QIAGEN, catalog number 79254). RNA samples were then sent to the Molecular Resource Center at the University of Tennessee Health Science Center for microarray analysis using the Affymetrix Clariom S. Data is represented as the fold change of RNA in the IIA+ BAT relative to C57BL/6 BAT.

\section{Results}

\section{IIA+ Energy Expenditure at Thermoneutrality}

My initial experiments were designed to discover whether PLA2G2A expression resulted in elevated thermogenesis not only at room temperature $\left(22^{\circ} \mathrm{C}\right)$ but also in the thermoneutral zone $\left(28^{\circ} \mathrm{C}\right)$. While both the $\mathrm{BL} / 6$ and IIA + mice had decreased thermogenesis at thermoneutral conditions, the IIA+ animals clearly had elevated VO2, VCO2, and energy expenditure at both ambient and thermoneutral temperatures (Figure 4-1A through C). There were no significant changes in respiratory exchange ratio (RER) between the C57BL/6 and IIA+ mice at room temperature. However, there was a substantial decrease in the RER during the dark cycle of the IIA+ animals at thermoneutrality ( $\sim .83)$ compared to the RER at ambient temperature ( .93$)$, indicating an increased utilization of fats at higher temperatures (Figure 4-1D). Also, there were no changes in physical activity in any of the conditions between the groups signifying that the increased thermogenesis seen in the IIA+ animals was not due to more activity (data not shown). 

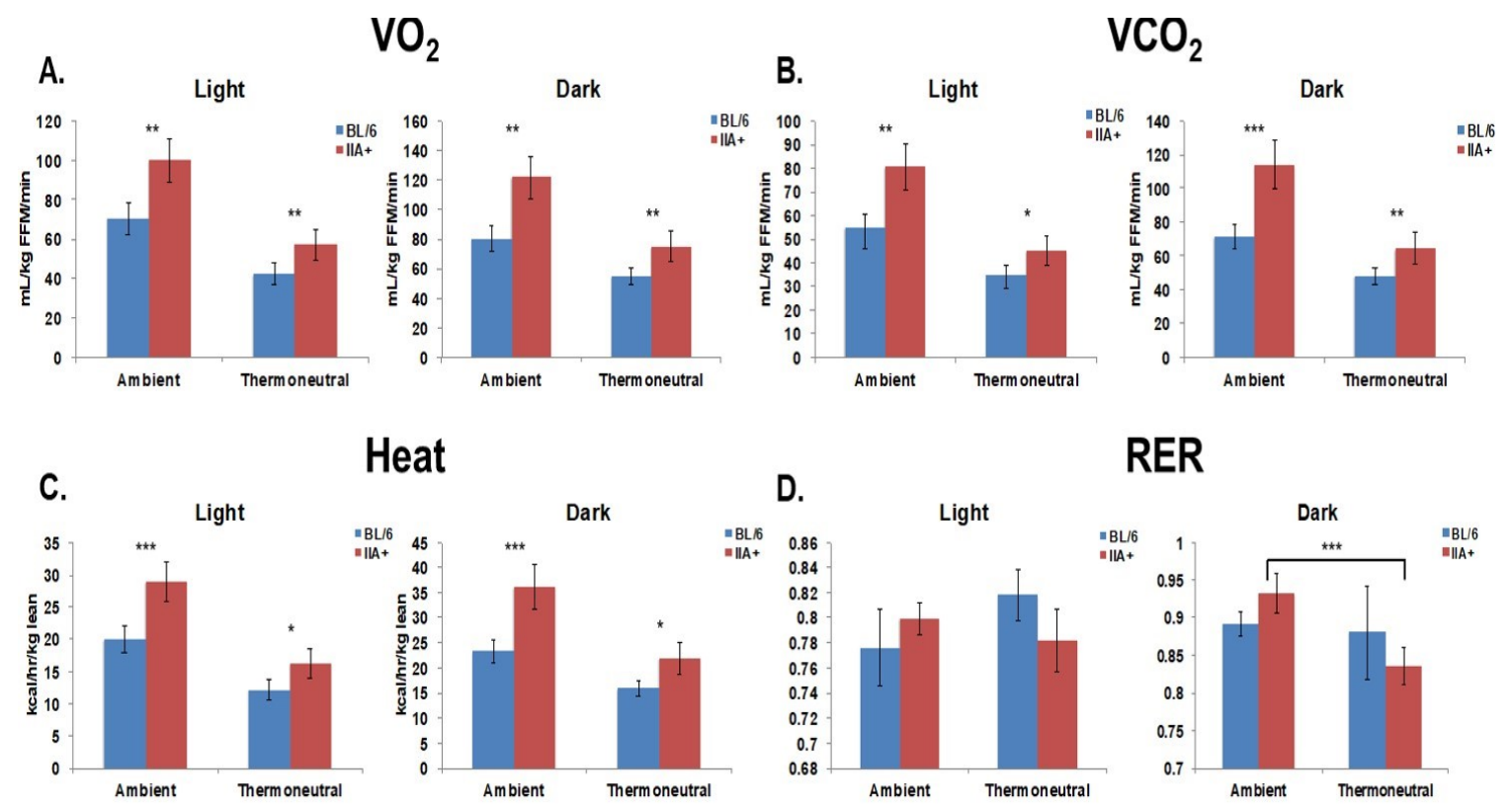

Heat
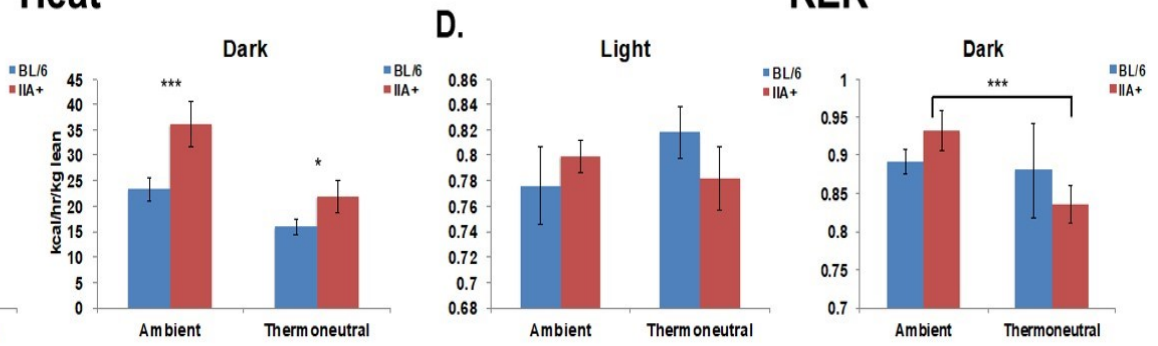

Figure 4-1. IIA+ mice have elevated energy expenditure at thermoneutrality

12 week old C57BL/ 6 or IIA+ mice were placed individually in CLAMS chambers and fed a chow diet for 1 week. Various metabolic measurements at either ambient $\left(22^{\circ} \mathrm{C}\right)$ or thermoneutral temperature $\left(28^{\circ} \mathrm{C}\right)$ were assessed. A, B) $\mathrm{VO} 2$ and $\mathrm{VCO} 2$ at ambient/thermoneutrality in Light or Dark cycles are expressed as $\mathrm{ml} / \mathrm{kg}$ lean mass $/ \mathrm{hr}$ of $\mathrm{O} 2$ or $\mathrm{CO} 2$ consumption. C) Energy expenditure (heat production) is expressed as $\mathrm{kcal} / \mathrm{hr} / \mathrm{kg}$ fat free mass. D) Respiratory exchange quotient (RER) was determined for both groups of mice at each temperature. Each bar is the average measurement from 6 mice. Data was analyzed by ANCOVA, using lean mass as the covariate and each metabolic measurement as the dependent variable. ${ }^{*} p<0.05, * * p<0.01, * * * p<0.001$. 


\section{Citrate Synthase Activity in BAT, WAT, and Muscle}

Citrate synthase activity is a common measure of mitochondrial content in thermogenic tissues such as BAT or skeletal muscle (177). We found that PLA2G2A increased citrate synthase activity in the BAT of IIA+ mice compared to C57BL/6 BAT (Figure 4-2A, B). The elevated VO2 and energy expenditure from the metabolic cages prompted us to measure citrate synthase activity in the skeletal muscle as well since it is a major oxidative organ that can contribute to thermogenesis. There were no differences in citrate synthase activity between the skeletal muscle of C57BL/6 or IIA+ mice (Figure 4-2C, D). These data suggest that PLA2G2A improves thermogenesis due to enhanced mitochondrial respiration in BAT.

\section{Mitochondrial Complex Protein Expression in BAT}

To further characterize how PLA2G2A was affecting adipose tissue thermogenesis, we assessed protein levels of the 5 mitochondrial complexes responsible for oxidation phosphorylation (Figure 4-3A, B). IIA+ BAT had a significant induction in mitochondrial complex components compared to the C57BL/6 BAT, which expresses no PLA2G2A protein (Figure 4-3A, C). Since we observed elevated VO2 in the white adipocytes by Seahorse analysis (Figure 4-4F through I), we were also interested in mitochondrial oxidative phosphorylation protein expression in WAT. However, the only mitochondrial complex induced in the WAT of IIA+ mice was complex V (ATP5A), and there was no change in the other proteins (Figure 4-3B, D).

\section{Improved Mitochondrial Respiration in IIA+ Adipocytes}

Given the enhanced metabolic phenotype of the IIA+ mice, we tested whether the mitochondrial respiration in isolated brown adipocytes of the IIA + animals was elevated using the Seahorse metabolic analyzer. With the mitochondrial stress test, brown and white subcutaneous adipocytes from IIA+ mice showed considerably higher basal VO2 compared to brown adipocytes from C57BL/6 animals (Figure 4-4A, B, F through G). Maximal respiration, which is calculated following the addition of the uncoupling agent FCCP, was significantly higher in the IIA+ brown and white adipocytes as well (Figure 4-4C, H). There was a dramatic increase in the proton leak in the IIA+ adipocytes as well, suggesting an induction in mitochondrial uncoupling in the IIA+ brown and white adipocytes (Figure 4-4D, I). Interestingly, non-mitochondrial oxygen consumption rates were similar in the BL/6 and IIA+ brown adipocytes, but almost doubled in the $\mathrm{IIA}+$ white adipocytes compared to BL/6 white adipocytes.

\section{IIA+ Adipocyte Protein Expression}

My next experiments tested whether thermogenic markers involved in the upregulation of mitochondrial uncoupling were altered in the brown adipocytes of from 

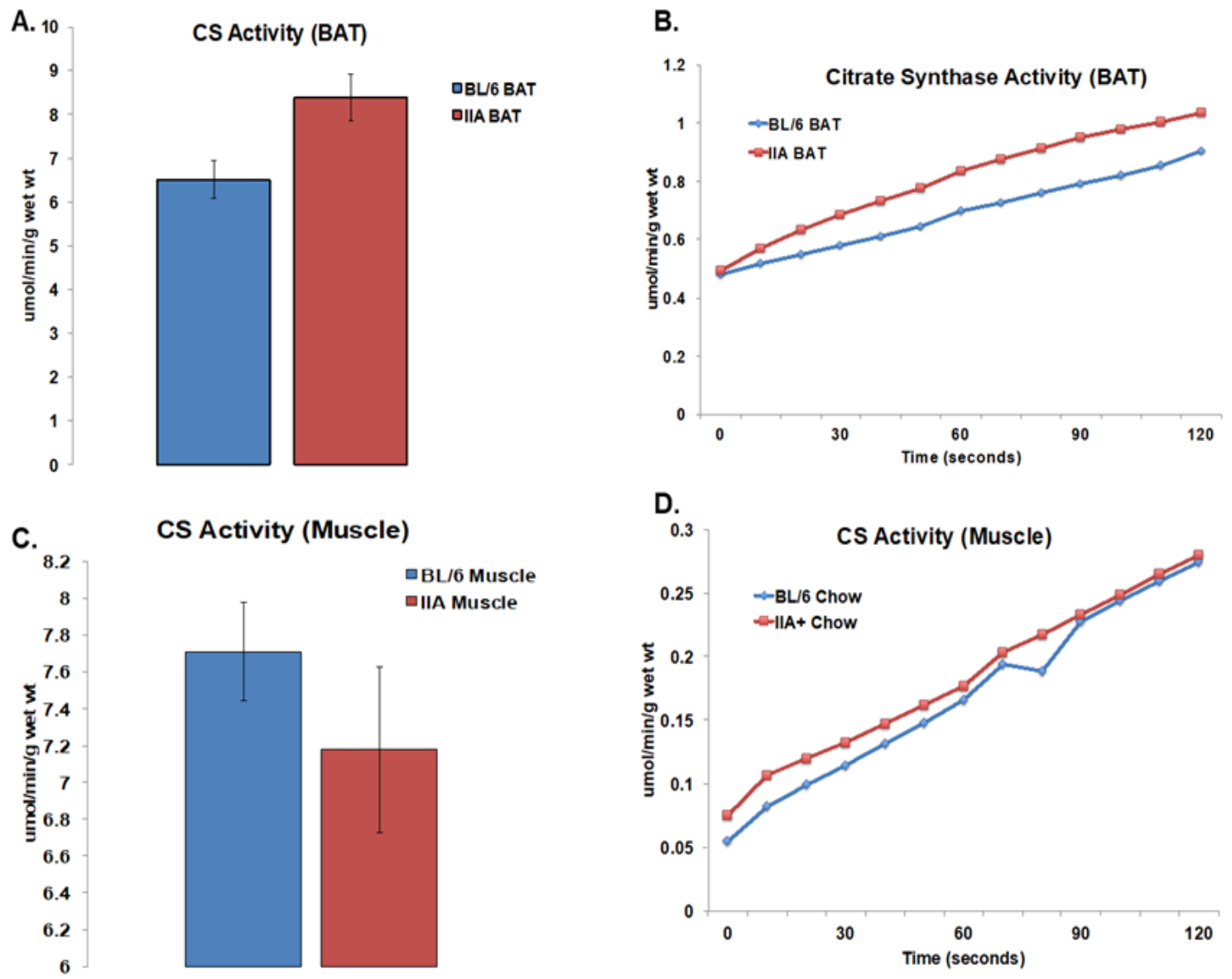

Figure 4-2. Citrate synthase activity increased in IIA+ BAT

BAT and skeletal muscle were harvested from 18 week old mice and homogenized using a citrate synthase activity assay buffer described in methods. CS activity was measured by detecting the change in absorbance per minute of DTNB on a plate reader at $412 \mathrm{~nm}$ following the addition of oxaloacetate. Acetyl CoA was added to the samples after time 0 was measured and the OD512 was measured every 30 seconds over the following 2 minutes. A) CS activity expressed as umol $/ \mathrm{min} / \mathrm{g}$ wet weight of BAT. B) Line plot showing CS activity of BAT over 2 minutes following acetyl-CoA addition. C) CS activity expressed as umol $/ \mathrm{min} / \mathrm{g}$ wet weight of skeletal muscle. D) Line plot showing CS activity of skeletal muscle over 2 minutes following Acetyl CoA addition. ${ }^{*} \mathrm{p}<0.05$, $* * \mathrm{p}<0.01, * * * \mathrm{p}<0.001$. 
A.

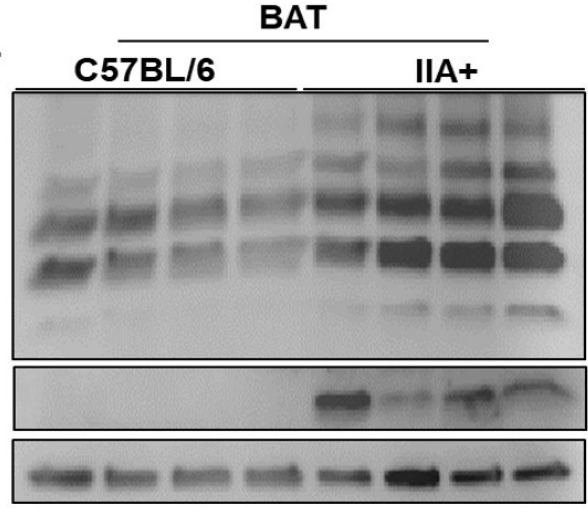

C.

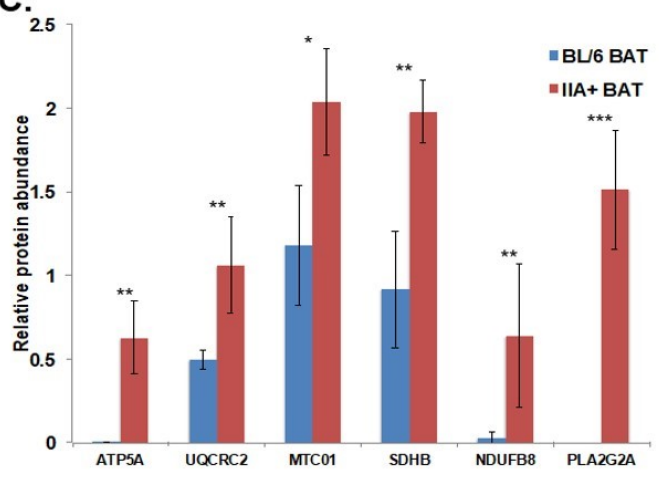

B.

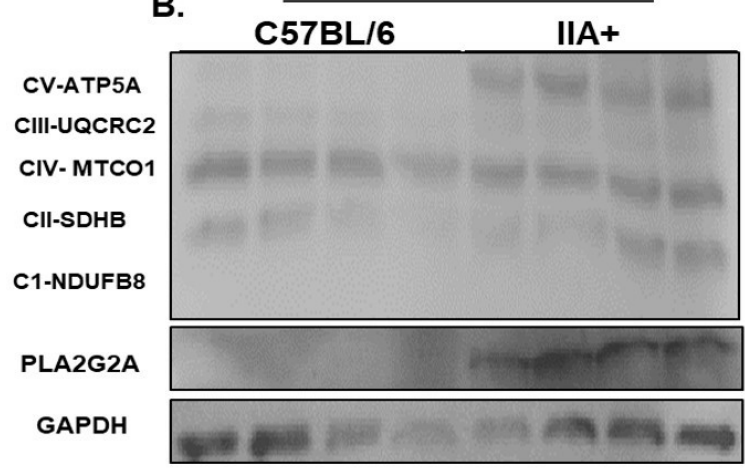

D.

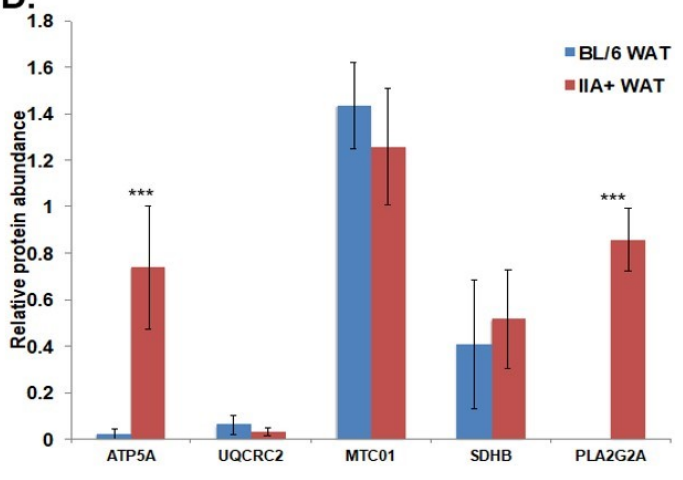

Figure 4-3. Mitochondrial complex proteins induced in IIA+ mice

Proteins were collected from the BAT of 4 C57BL/6 and 4 IIA + mice. An antibody for all 5 mitochondrial complex proteins involved in oxidative phosphorylation was used and representative westerns are shown. A) Western blots of BAT from C57BL/6 and IIA+ mice showing complex V (ATP5A), complex III (UQCRC2), complex IV (MTCO1), complex II (SDHB), complex 1 (NDUFB8), PLA2G2A and GAPDH. B) Western blots of iWAT from both animal groups displaying the same protein panel. C, D) The western blots were quantified and the resulting bar graphs are shown. The data are expressed as relative protein abundance. Each point is the average protein expression of 4 animals. The data were analyzed by independent samples T-test. ${ }^{*} \mathrm{p}<0.05, * * \mathrm{p}<0.01,{ }^{*} * \mathrm{p}<0.001$. 

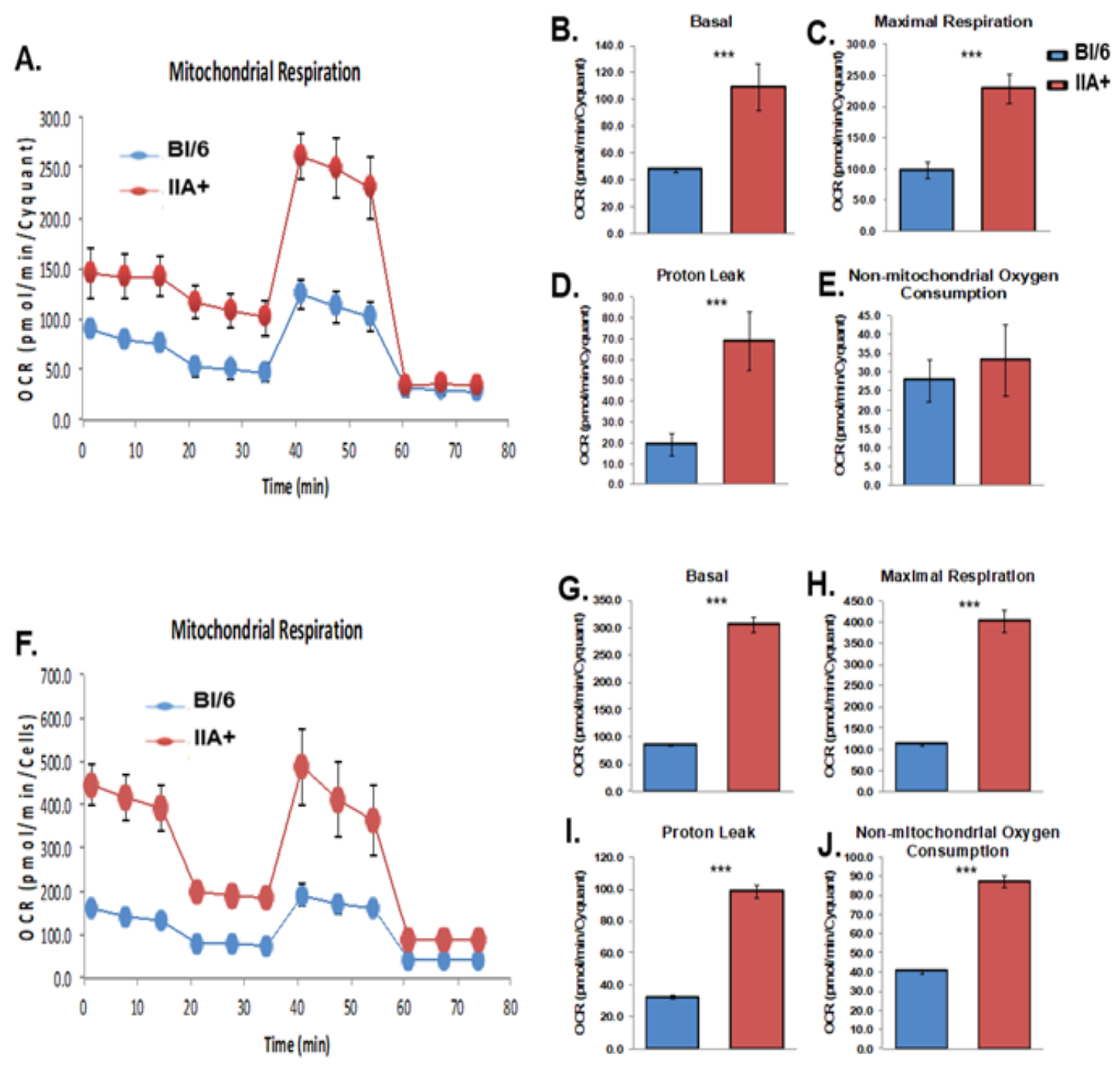

Figure 4-4. Mitochondrial respiration elevated in IIA+ white and brown adipocytes

Mature BL/6 and IIA+ brown (A-E) or inguinal white (F-J) adipocytes were plated on 96 well Seahorse plates. A mitochondrial stress test was run on the Xfe96 bioanalyzer. Adipocyte preparations were derived from the pooled WAT or BAT of 5 animals. The adipocytes were differentiated in $10 \mathrm{~cm}$ dishes and then transferred to 96 well plates designated for the seahorse Xfe 96 bioanalyzer at 20,000 cells/well for analysis. There were 12 replicate wells for each preparation of adipocytes. Oligomycin, FCCP $(2 \mu \mathrm{M})$, and rotenone were added to the cells at 15,35 , and 55 minutes. Oxygen consumption rate (OCR) over the 75 minutes is shown for brown adipocytes (A) and white adipocytes (F). Basal respiration (B, G), Maximal respiration $(\mathrm{C}, \mathrm{H})$, mitochondrial proton leak (D, I) and non-mitochondrial oxygen consumption (E, J) are displayed for brown/white adipocytes. The data are expressed as OCR in $\mathrm{pmol} / \mathrm{min} / \mathrm{mitochondrial} \mathrm{DNA}$. The data were analyzed by independent T-test. ${ }^{*} \mathrm{p}<0.05,{ }^{* *} \mathrm{p}<0.01,{ }^{* * *} \mathrm{p}<0.001$. 
IIA+ mice. UCP1, SIRT1, and PGC-1 $\alpha$ were markedly induced in the IIA+ brown adipocytes (Figure 4-5A, B). Previous work found that AMPK signaling contributes to the induction of BAT thermogenesis and that AMPK stimulates fatty acid efflux from tissue, increases glucose transport and fatty acid oxidation, and reduces cytokine secretion in adipocytes $(178,179)$. We observed significant induction of phosphorylatedAMPK (Thr172) in the IIA+ adipocytes with no change in the total AMPK levels, indicating elevated AMPK signaling (Figure 4-5A, B). Moreover, immunostaining of C57BL/6 (Figure 4-5C) and IIA+ adipocytes (Figure 4-5C) showed a clear increase in $\mathrm{UCP} 1$ abundance and mitochondria as assessed by mito tracker in the IIA+ adipocytes (Figure 4-5C).

\section{Recombinant PLA2G2A Enzyme Improves Thermogenic Protein Expression in C57BL/6 Adipocytes}

The goal of the next experiments was to determine if the addition of purified PLA2G2A to non-expressing C57BL/6 brown adipocytes would induce thermogenic protein expression. Addition of PLA2G2A increased UCP1 and PRDM16 abundance in the C57BL/6 adipocytes after 24 hours of exposure (Figure 4-7). These results suggest that circulating PLA2G2A is sufficient to activate BAT.

\section{Changes in Substrate Transport and Chemokine Production in IIA+ BAT}

To assess differences in the BAT transcriptome of IIA+ and C57BL/6 mice, we conducted RNA microarray analysis of 5 brown adipose depots from each animal group. One of the most significantly up-regulated gene families based on the array was the solute carrier $(S l c)$ family, which comprises membrane transport proteins of various substrates, including mitochondrial carriers, along with fatty acid and glucose transporters (Figure 4-6A). Of the 17 Slc genes induced, the long chain fatty acid transport protein 1(Slc27al) and the very long chain acyl-CoA synthase (Slc27a2) are directly involved in long chain fatty acid (LCFA) and/or very-long chain fatty acid (VLCFA) transport and activation, suggesting enhanced fatty acid transport in the IIA+ adipocytes (180). Another subset of Slc genes, Slc25a34 and Slc25a51, belong to a family of nuclear-encoded transporters embedded in the inner mitochondrial membrane (Figure 4-6A) (181). In addition to the changes in the Slc family, we also observed significant down-regulation of numerous pro-inflammatory chemokines in the IIA+ BAT, including C-X-C motif ligand 13 (CXCL13), CXCL9, C-C motif ligand 21c (CCL21c), CCL21b, CCL11, chemokine like receptor-1 (CMKLR-1) (Figure 4-6B).

\section{Discussion}

My previous work showed that PLA2G2A expression protected mice from dietinduced obesity and insulin resistance, at least in part through elevated thermogenesis $(51,52)$. In this study, we define additional thermogenic changes in the WAT and BAT 

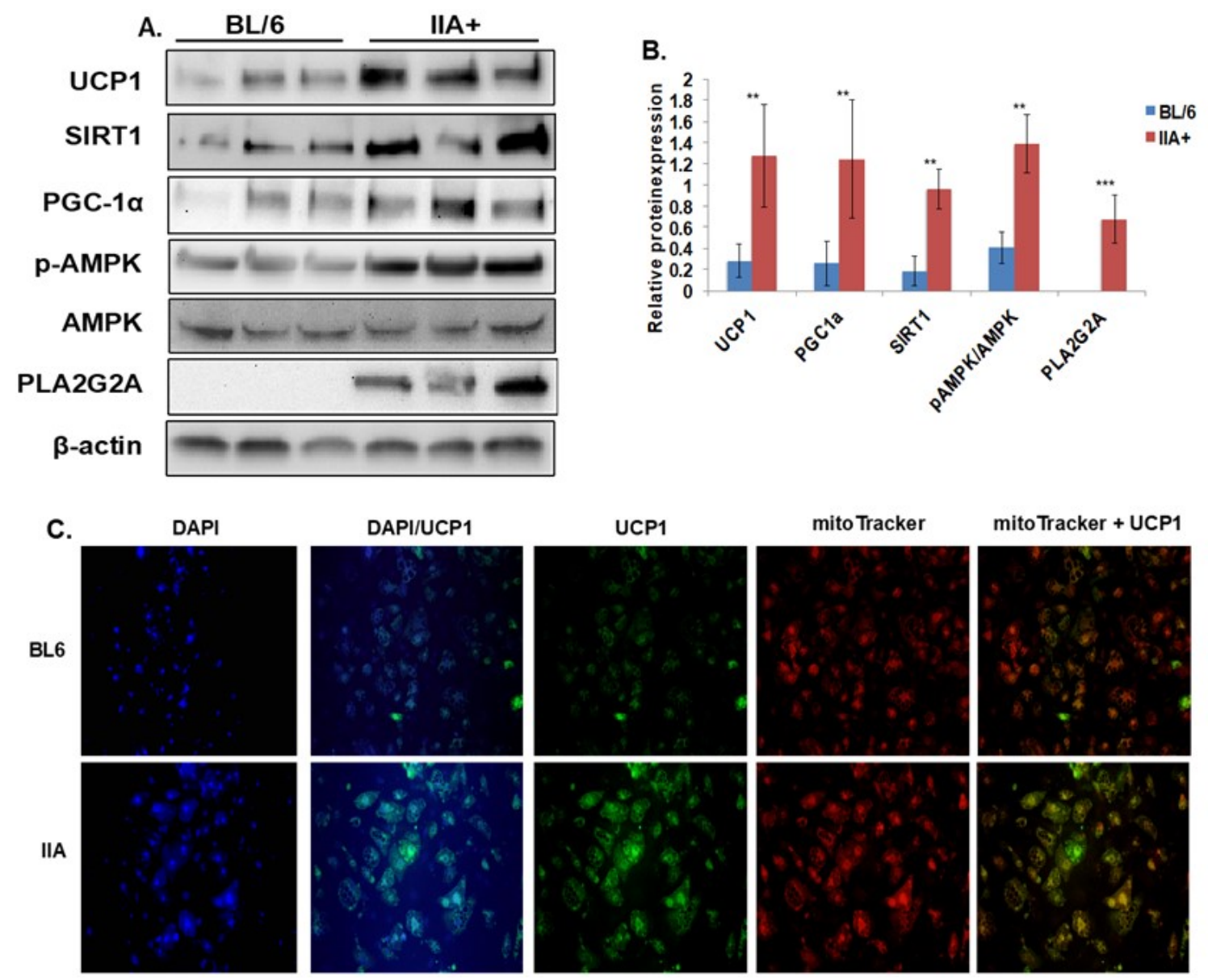

Figure 4-5. Thermogenic protein expression elevated in IIA+ mice

Brown adipocytes were prepared from $5 \mathrm{BL} / 6$ and $5 \mathrm{IIA}+\mathrm{BAT}$ mice. Mature adipocytes were harvested for protein and the resulting western blots for UCP1, SIRT1, PGC-1 $\alpha$, pAMPK, AMPK, PLA2G2A and beta-actin were quantified and shown in panels A and B. C) Immunohistochemistry of BL/6 and IIA+ adipocytes with DAPI, DAPI/UCP1, UCP1, mitoTracker, or mitoTracker/UCP1 staining. The data were analyzed by one-way ANOVA. ${ }^{*} \mathrm{p}<0.05,{ }^{* *} \mathrm{p}<0.01, * * * \mathrm{p}<0.001$. 

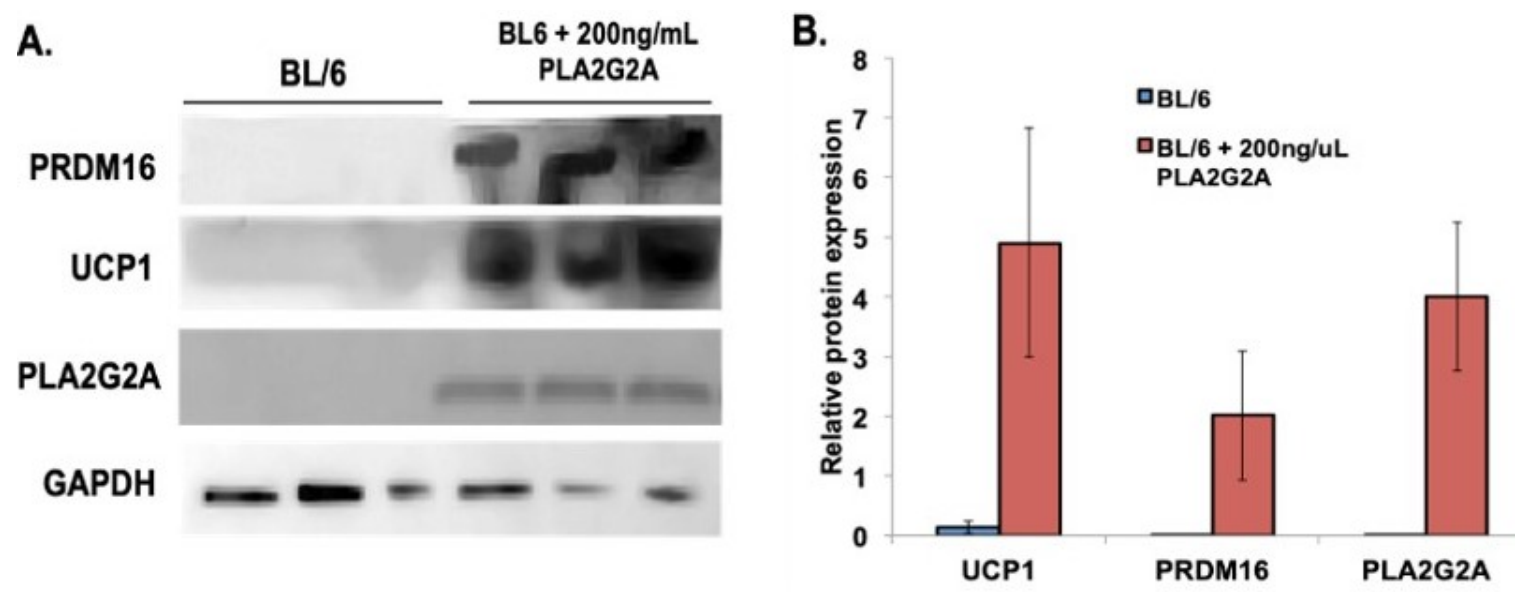

Figure 4-6. Addition of PLA2G2A to brown adipocytes increases UCP1 expression

Brown adipocytes were prepared from 3 groups of 5 BL/6 mice. Mature adipocytes were treated with recombinant PLA2G2A enzyme for 24 hours and then harvested for western blot analysis. Protein expression of UCP1, PRMD16, PLA2G2A, and GAPDH were quantified and shown in A and B. The data were analyzed by independent t-test. $* p<0.05$, $* * \mathrm{p}<0.01, * * * \mathrm{p}<0.001$.
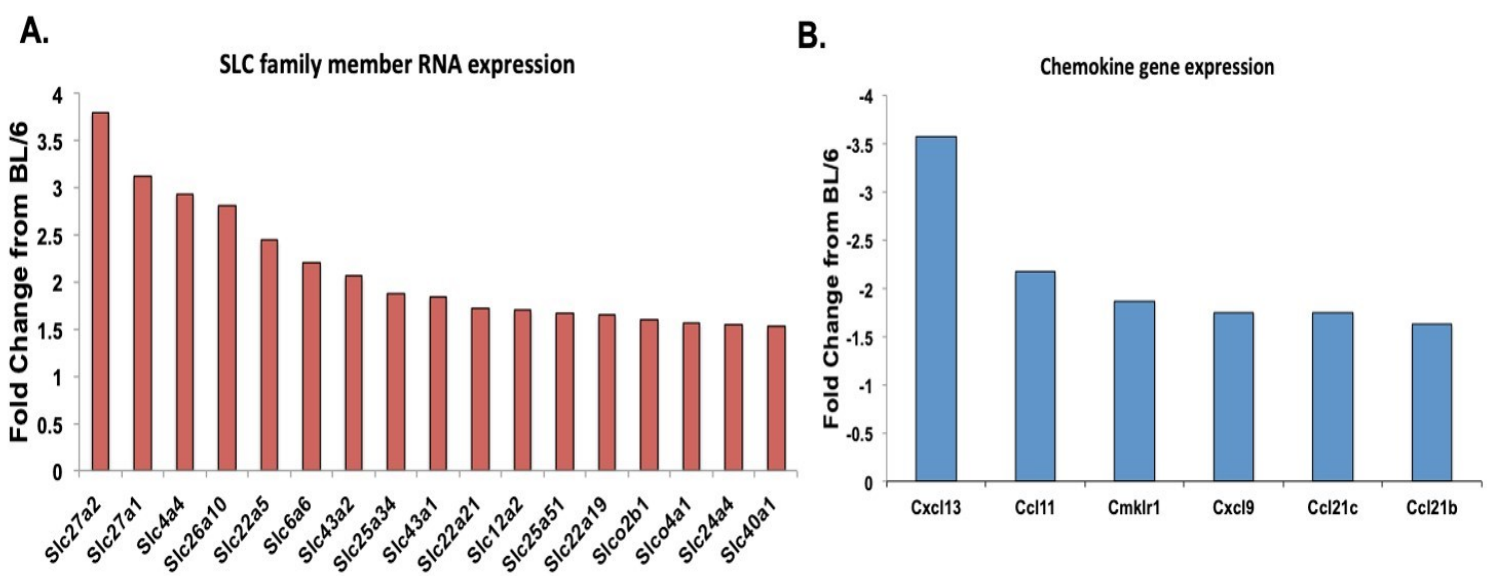

Figure 4-7. Changes in BAT transcriptome in the IIA+ mice

BAT was collected from $5 \mathrm{BL} / 6$ and 5 IIA+ mice and used for RNA microarray analysis. A) 17 family members of the Slc family induced at least 1.5 fold in the IIA+ mice. B) Chemokine genes significantly down-regulated in the IIA+ BAT. Each gene was significantly up or down-regulated in the IIA+ mice 1.5 fold with a p-value less than 0.05 . 
in response to expression or addition of PLA2G2A.. PLA2G2A expression increased VO2 and energy expenditure even at thermoneutrality demonstrating that the mice have elevated metabolism independent of environment. Citrate synthase activity, a common marker of mitochondrial content in metabolic tissues, was elevated in the BAT of IIA+ mice but unchanged in the skeletal muscle. Direct administration of recombinant PLA2G2A enzyme to C57BL/6 brown adipocytes, which do not express PLA2G2A, increased thermogenic gene expression of UCP1. Lastly, microarray analysis of BAT from IIA + mice strongly suggests the induction of substrate transport, particularly fatty acids and glucose, and the inhibition of pro-inflammatory chemokine production in BAT.

The major regulator of mammalian non-shivering thermogenesis is BAT primarily due to mitochondrial expression of UCP1, which functions by uncoupling oxidative phosphorylation from ATP synthesis to elevate energy expenditure $(87,182)$. Unsurprisingly, temperature plays a significant role in UCP1 expression and the activation of BAT thermogenesis. Numerous studies have shown that cold exposure induces BAT-specific non-shivering thermogenesis in both rodents and humans, likely through enhanced adrenergic signaling $(154,183,184)$. Conversely, other experiments have found BAT activity is diminished in thermoneutral conditions $(185,186)$. In my study, we did observe a decrease in $\mathrm{VO} 2, \mathrm{VCO} 2$ and energy expenditure in both animal groups in response to thermoneutrality (Figure 4-1A through F). However, even at the thermoneutral temperature the IIA+ mice displayed elevated thermogenesis relative to C57BL/6 mice, indicating that PLA2G2A expression stimulates metabolism (Figure 4-1A through F). The respiratory exchange ratio (RER), a measure of fuel substrate preference, significantly declined in the IIA+ mice at thermoneutrality, indicating enhanced fatty acid oxidation (Figure 4-1G, H).

Fatty acid oxidation which is required for BAT thermogenesis involves the carnitine palmitoyltransferase enzymes CPT1 and CPT2 $(187,188)$. These enzymes mediate mitochondrial long chain fatty acid beta-oxidation through consecutive carnitine acyltransferase reactions allowing the translocation of long chain acyl-CoAs across the mitochondrial membranes. Although we did not observe elevated Cpt $1 b$ mRNA in the RNA arrays, there was an increase in Slc27a2 which generates fatty-acyl-CoA for translocation across mitochondrial membranes. Moreover, many genes involved in fatty acid oxidation were also induced in the IIA + BAT, including enoyl-CoA hydratase and 3hydroxyacyl CoA dehydrogenase (Ehhadh, 2.8-fold), carboxylesterase 1f (Ces 1f, 2.2fold), acyl-coenzyme A thioesterase 1 (Acot1, 1.73-fold), and long-chain acyl-CoA synthetase-1 (Acsl1, 1.6-fold). Fatty-acyl-CoAs are required for BAT thermogenesis, and mice with deficiencies in acyl-CoAs synthetases (ACSLs) have reduced oxygen consumption, fatty acid oxidation and are cold intolerant (189). We postulate that since the IIA+ animals still have elevated energy expenditure at thermoneutrality due to enhanced BAT activation, that the fatty acid oxidation remains induced in the IIA + mice even at warmer conditions, thus driving the lower RER.

In addition to BAT, skeletal muscle is one of the major determinants of metabolic rate and comprises approximately $45-55 \%$ of the total mammalian body weight (190- 
192). Skeletal muscle has long been known for its ability to regulate body temperature by shivering. However, recent research suggests that it plays a key role in non-shivering thermogenesis as well in an UCP1 independent manner through a futile sarcoplasmic reticulum calcium ATPase pump known as SERCA $(193,194)$. Since skeletal muscle is a major element in whole body VO2 and energy expenditure, we examined whether it contributed to the enhanced thermogenic phenotype in the IIA+ mice. One method for assessing this question is to measure citrate synthase activity, a validated biomarker for measuring mitochondrial density and cellular oxidative capacity in both skeletal muscle and BAT $(177,195,196)$. There were no changes in skeletal muscle citrate synthase activity between the C57BL/6 and IIA+ mice (Figure 4-2C through D), but a clear induction in citrate synthase activity was seen in the IIA+ BAT (Figure 4-2A, B). Western blots from IIA+ BAT revealed a significant increase in mitochondrial oxidative phosphorylation complexes (Figure 4-3A). In brown adipocytes from IIA+ mice, thermogenesis was also induced based on the Seahorse mitochondrial stress test (Figure 4-4A through D), as well as protein expression of UCP1, PGC-1 $\alpha$, SIRT1, and phosphorylated AMPK (Figure 4-5). Moreover, it is understood that BAT and UCP1mediated thermogenesis induce a mitochondrial proton leak that contributes highly to BAT basal oxygen consumption $(197,198)$. The proton leak in IIA+ brown adipocytes was 3-fold higher than the C57BL/6 adipocytes (Figure 4-4D). Thermogenic protein expression was significantly induced in the C57BL/6 brown adipocytes following addition of recombinant PLA2G2A enzyme (Figure 4-6). Taken together, these data indicate that PLA2G2A stimulates the metabolic rate through up-regulation of UCP1mediated adipose tissue thermogenesis and not through skeletal muscle.

The 'browning' or beiging of WAT, which is morphologically similar to BAT may contribute to the protection against obesity as well (199-201). While we did not observe any change in citrate synthase activity in the WAT between C57BL/6 and IIA+ mice (Figure 4-2A, B), there was a significant induction in oxygen consumption, basal and maximal metabolic rate, and mitochondrial proton leak in the IIA+ white adipocytes (Figure 4-4F through J). Likewise, protein levels of complex 5 of the mitochondrial respiratory chain (ATP5A) were induced as well (Figure 4-3B), and we have previously reported that IIA+ adipocytes from WAT have elevated thermogenic protein expression (51). These data suggest that PLA2G2A improves white adipose tissue thermogenesis, which likely contributes to the improved metabolic phenotype of the IIA + animals.

Metabolically active BAT utilizes high amounts of readily available energy, especially glucose and fatty acids, to impact the metabolic rate. Cold exposure or $\beta$ adrenergic stimulation improve glucose and fatty acid uptake in BAT via differentially regulated substrate transport genes in rodents and humans (202-204). RNA microarray analysis of IIA+ and C57BL/6 BAT revealed a clear induction in substrate transport genes, particularly of the Slc family, in the IIA+ mice. As stated earlier, fatty acids are required for UCP1-mediated uncoupling in BAT (125). The mRNA levels of Slc27al which is a LCFA transporter and Slc27a2 which is the initiating step in fatty acid translocation into the mitochondria were induced over 3-fold in the IIA + BAT (Figure 4-7A). Members of the Slc25 sub-family, Slc25a34 and Slc25a51, which are mitochondrial ion transporters were also elevated in the IIA+ BAT (181). There was an 
induction of the mRNA of the Slc22 family members, which are involved in the transport of various ions and endogenous substrates such as L-carnitine and prostaglandins (205). Previous reports have shown L-carnitine transport in BAT is elevated in response to cold (204). With regard to prostaglandin transport, PLA2G2A releases fatty acids following cleavage of lysophospholipids, and one potential fatty acid candidate is arachidonic acid. Release of arachidonic acid is a key step in prostaglandin synthesis $(109,206)$. Interestingly, previous studies have shown that activation of prostaglandin E receptor 4 (EP4) via binding of prostaglandin E2 (PGE2) is antiinflammatory in mice, and array data from the IIA+ BAT showed a 1.7-fold induction in EP4 mRNA expression (data not shown). Altogether, these data indicate that substrate transport expression is elevated in the BAT of IIA + mice to fuel increased energy demands. Additionally, PLA2G2A increases release of fatty acids that may promote PGE2 synthesis and activation of EP4 in BAT, fostering an anti-inflammatory state.

The mRNA levels of pro-inflammatory chemokines were also reduced in the IIA+ BAT (Figure 4-7B). It is known that macrophages and immune cells are present in BAT and play a role in adipose tissue thermogenesis $(207,208)$. Pro-inflammatory cytokines such as TNF $\alpha$, CCL2 2 and CCL5 are up-regulated in the BAT of obese mice and result in suppression of UCP1 expression and mitochondrial respiration (209). In vitro studies indicate that addition of TNF $\alpha$ to brown adipocytes causes insulin resistance via phosphorylation and inhibition of insulin receptor substrate 2 (IRS2) (210). Importantly, many of the genes encoding chemokines that were down-regulated in the IIA+ BAT (CXCL13, CXCL9, CCL21c, CCL11, etc.) (Figure 4-7B) are positively regulated by TNF $\alpha$ and likely contribute to a pro-inflammatory state (211). This suggests that PLA2G2A promotes an anti-inflammatory state in BAT, possibly due to enhanced EP4 expression. The anti-inflammatory state favors activation of BAT, thus increasing glucose and fatty acid utilization by the tissue in order to improve insulin sensitivity, lipolysis, and ultimately protection from obesity and insulin resistance. 


\section{CHAPTER 5. CONCLUSION}

\section{Summary and Future Directions}

In conclusion, we discovered that expression of human PLA2G2A in mice protects them from diet-induced obesity. IIA + mice displayed significantly increased energy expenditure and oxygen consumption, which was primarily due to enhanced BAT thermogenesis and beiging of WAT. Adipose tissue thermogenesis was confirmed in isolated adipocytes, as there was marked induction in thermogenic protein expression and mitochondrial proton leakage in both brown and white adipocytes isolated from IIA+ animals. Similarly, the IIA+ mice had elevated energy expenditure even in thermoneutral conditions. The BAT from IIA+ mice had increased expression of numerous genes involved in substrate transport, including glucose and fatty acids, suggesting that there is increased substrate utilization by the adipose in order to fuel adipose tissue thermogenesis. This increased substrate transport in BAT may be one mechanism by which the IIA+ mice showed elevated glucose tolerance and insulin sensitivity as well, as the utilization of glucose by BAT may reduce total blood glucose levels. Finally, an interesting relationship exists between PLA2G2A expression and thyroid hormone status. Although PLA2G2A increases basal metabolic rate, it was unable to counteract reductions in metabolism commonly associated with hypothyroidism. Conversely, PLA2G2A and elevated thyroid hormone levels displayed a synergistic relationship and dramatically increasing insulin sensitivity and glucose tolerance in mice, at least in part due to enhanced GLUT4 expression in BAT. Altogether, these data suggest that PLA2G2A has a beneficial effect on adipose tissue thermogenesis and whole-body metabolism and can counteract obesity and symptoms of Type 2 diabetes including insulin resistance and glucose intolerance.

\section{Knockout of Pla2g2a in Mice}

The C57BL/6 mice used throughout these experiments do not express endogenous Pla2g2a due to a frameshift mutation in exon 3 of the Pla2g2a gene (16). Because of this, the majority of the metabolic experiments were done on mice that overexpress the human PLA2G2A. One important issue that needs to be addressed is whether knock-out of the endogenous Pla2g2a gene will reduce metabolic rate or increase susceptibility to weight gain. Since the C57BL/6 mice are a natural Pla2g2a knockout, one strategy to assess the role of endogenous murine Pla2g2a in metabolic regulation would be to introduce the correct Pla2g2a gene by backcrossing BALB/c mice, which express endogenous Pla2g2a, with C57BL/6 animals to introduce the Pla2g2a gene on a C57BL/6 background. This animal model will provide meaningful information on whether the endogenous murine Pla2g2a has similar effects as human PLA2G2A on adipose tissue thermogenesis and insulin signaling. The other approach would be to repair the murine Pla2g2a gene with CRISPR/Cas9 in the C57BL/6 mice. 


\section{Impact of $\mathbf{S P L A}_{2}$ Knockdown in Brown and White Adipocytes}

A second important question would be how other $\mathrm{sPLA}_{2} \mathrm{~s}$ will compensate when Pla2g2a, Pla2g5, or Pla2g2e are knocked down in brown adipocytes. One approach would be to generate brown and white adipocytes from wild-type and Pla2g2a KO mice and analyze the compensatory expression of the $\mathrm{sPLA}_{2} \mathrm{~s}$, along with the metabolic effects on oxygen consumption and energy expenditure.

\section{Tissue-specific Effect of PLA2G2A Expression in the Liver and BAT}

Human PLA2G2A expression in mice results in dramatic improvements in insulin signaling and glucose tolerance along with increased expression of many proteins involved in insulin signaling (Figure 2-3, 3-4, and 3-8). Interestingly, PLA2G2A is widely expressed, but it has particularly high abundance in the liver and adipose depots of IIA + mice (Figure 2-5). Another significant question is whether the metabolic effects of PLA2G2A are mediated by its secretion from the liver or BAT. We have shown that the expression of PLA2G2A in brown adipocytes was sufficient to stimulate oxygen consumption and mitochondrial uncoupling (Figure 4-4). To test this, future studies should assess the impact of tissue-specific expression of PLA2G2A in the liver and/or BAT. Liver-specific expression will determine if hepatic PLA2G2A secretion activates BAT thermogenesis and whole-body metabolism. With regard to BAT-specific expression, introducing PLA2G2A specifically into BAT will determine if the increase in BAT mitochondrial uncoupling and thermogenesis is an autocrine effect, and show whether or not PLA2G2A in BAT alone is sufficient to alter insulin sensitivity and weight gain as was observed in Figure 2-1 and Figure 3-2.

\section{Identification of Lysophospholipids and FFAs in IIA+ BAT}

The final key issue which needs to be addressed is which lipid products of PLA2G2A are mediating the metabolic effects. The enzymatic function of PLA2G2A is to release lysophospholipids and fatty acids, many of which, such as EPA, DHA or AA have profound metabolic roles. AA in particular can be further modified to prostaglandins, leukotrienes and HETEs. The lysophospholipids can generate LPA and PAF. The identification of metabolically active lipids released by PLA2G2A activity in BAT may provide mechanistic insight on why the IIA+ mice have such high basal oxygen consumption and metabolic rate. Future studies could use lipidomics to identify which lipids are elevated in the BAT of IIA + mice. If there are any marked changes in lipid profiles between the BAT of C57BL/6 and IIA+ mice, these lipids can be added to isolated brown adipocytes and their oxygen consumption and expression of thermogenic proteins can be measured to determine if direct addition of specific lipids mediates adipose tissue thermogenesis. 


\section{LIST OF REFERENCES}

1. Makoto Murakami, Y. T., Christophe Giard, Kei Yamamoto, Gerard Lambeau. (2010) Emerging roles of secreted phospholipase A2 enzymes: Lessons from transgenic and knockout mice. Biochimie 92, 561-582

2. Murakami, M., Taketomi, Y., Miki, Y., Sato, H., Hirabayashi, T., and Yamamoto, K. (2011) Recent progress in phospholipase A2 research: From cells to animals to humans. Progress in Lipid Research 50, 152-192

3. Murakami, M., Taketomi, Y., Sato, H., and Yamamoto, K. (2011) Secreted phospholipase A2 revisited. The Journal of Biochemistry 150, 233-255

4. Murakami, M., Sato, H., Miki, Y., Yamamoto, K., and Taketomi, Y. (2015) A new era of secreted phospholipase A(2). Journal of lipid research 56, 1248-1261

5. Murakami, M., Miki, Y., Sato, H., Murase, R., Taketomi, Y., and Yamamoto, K. (2018) Group IID, IIE, IIF and III secreted phospholipase A2s. Biochimica et Biophysica Acta (BBA) - Molecular and Cell Biology of Lipids

6. Pruzanski, W., and Vadas, P. (1988) Secretory synovial fluid phospholipase A2 and its role in the pathogenesis of inflammation in arthritis. The Journal of rheumatology 15, 1601-1603

7. van Meer, G., Voelker, D. R., and Feigenson, G. W. (2008) Membrane lipids: where they are and how they behave. Nature reviews. Molecular cell biology $\mathbf{9}$, 112-124

8. Grass, D. S., Felkner, R. H., Chiang, M. Y., Wallace, R. E., Nevalainen, T. J., Bennett, C. F., and Swanson, M. E. (1996) Expression of human group II PLA2 in transgenic mice results in epidermal hyperplasia in the absence of inflammatory infiltrate. The Journal of clinical investigation 97, 2233-2241

9. Menschikowski, M., Platzbecker, U., Hagelgans, A., Vogel, M., Thiede, C., Schonefeldt, C., Lehnert, R., Eisenhofer, G., and Siegert, G. (2012) Aberrant methylation of the M-type phospholipase $\mathrm{A}(2)$ receptor gene in leukemic cells. BMC Cancer 12, 576

10. Sakata, T., Nakamura, E., Tsuruta, Y., Tamaki, M., Teraoka, H., Tojo, H., Ono, T., and Okamoto, M. (1989) Presence of pancreatic-type phospholipase A2 mRNA in rat gastric mucosa and lung. Biochimica et biophysica acta 1007, 124126

11. Mallat, Z., Benessiano, J., Simon, T., Ederhy, S., Sebella-Arguelles, C., Cohen, A., Huart, V., Wareham, N. J., Luben, R., Khaw, K. T., Tedgui, A., and Boekholdt, S. M. (2007) Circulating secretory phospholipase A2 activity and risk of incident coronary events in healthy men and women: the EPIC-Norfolk study. Arterioscler Thromb Vasc Biol 27, 1177-1183

12. Rosenson, R. S. (2010) Phospholipase A2 inhibition and atherosclerotic vascular disease: prospects for targeting secretory and lipoprotein-associated phospholipase A2 enzymes. Current opinion in lipidology 21, 473-480

13. Boilard, E., Lai, Y., Larabee, K., Balestrieri, B., Ghomashchi, F., Fujioka, D., Gobezie, R., Coblyn, J. S., Weinblatt, M. E., Massarotti, E. M., Thornhill, T. S., Divangahi, M., Remold, H., Lambeau, G., Gelb, M. H., Arm, J. P., and Lee, D. M. (2010) A novel anti-inflammatory role for secretory phospholipase 
$\mathrm{A}<\mathrm{sub}>2</$ sub $>$ in immune complex-mediated arthritis. EMBO Molecular Medicine 2, 172-187

14. Kugiyama, K., Ota, Y., Takazoe, K., Moriyama, Y., Kawano, H., Miyao, Y., Sakamoto, T., Soejima, H., Ogawa, H., Doi, H., Sugiyama, S., and Yasue, H. (1999) Circulating levels of secretory type II phospholipase A(2) predict coronary events in patients with coronary artery disease. Circulation 100, 1280-1284

15. Ivandic, B., Castellani, L. W., Wang, X. P., Qiao, J. H., Mehrabian, M., Navab, M., Fogelman, A. M., Grass, D. S., Swanson, M. E., de Beer, M. C., de Beer, F., and Lusis, A. J. (1999) Role of group II secretory phospholipase A2 in atherosclerosis: 1. Increased atherogenesis and altered lipoproteins in transgenic mice expressing group IIa phospholipase A2. Arterioscler Thromb Vasc Biol 19, 1284-1290

16. Kennedy, B. P., Payette, P., Mudgett, J., Vadas, P., Pruzanski, W., Kwan, M., Tang, C., Rancourt, D. E., and Cromlish, W. A. (1995) A Natural Disruption of the Secretory Group II Phospholipase A2 Gene in Inbred Mouse Strains. Journal of Biological Chemistry 270, 22378-22385

17. Eckey, R., Menschikowski, M., Lattke, P., and Jaross, W. (2004) Increased hepatic cholesterol accumulation in transgenic mice overexpressing human secretory phospholipase A2 group IIA. Inflammation 28, 59-65

18. Ghesquiere, S. A., Gijbels, M. J., Anthonsen, M., van Gorp, P. J., van der Made, I., Johansen, B., Hofker, M. H., and de Winther, M. P. (2005) Macrophagespecific overexpression of group IIa sPLA2 increases atherosclerosis and enhances collagen deposition. Journal of lipid research 46, 201-210

19. Webb, N. R., Bostrom, M. A., Szilvassy, S. J., van der Westhuyzen, D. R., Daugherty, A., and de Beer, F. C. (2003) Macrophage-expressed group IIA secretory phospholipase A2 increases atherosclerotic lesion formation in LDL receptor-deficient mice. Arterioscler Thromb Vasc Biol 23, 263-268

20. Fraser, H., Hislop, C., Christie, R. M., Rick, H. L., Reidy, C. A., Chouinard, M. L., Eacho, P. I., Gould, K. E., and Trias, J. (2009) Varespladib (A-002), a secretory phospholipase A2 inhibitor, reduces atherosclerosis and aneurysm formation in ApoE-/- mice. Journal of cardiovascular pharmacology 53, 60-65

21. Nicholls, S. J., Kastelein, J. J., Schwartz, G. G., Bash, D., Rosenson, R. S., Cavender, M. A., Brennan, D. M., Koenig, W., Jukema, J. W., Nambi, V., Wright, R. S., Menon, V., Lincoff, A. M., and Nissen, S. E. (2014) Varespladib and cardiovascular events in patients with an acute coronary syndrome: the VISTA-16 randomized clinical trial. Jama 311, 252-262

22. Talmud, P. J., and Holmes, M. V. (2015) Deciphering the Causal Role of sPLA2s and Lp-PLA2 in Coronary Heart Disease. Arterioscler Thromb Vasc Biol 35, 2281-2289

23. Shuvalova, Y. A., Khasanova, Z. B., Kaminnaya, V. I., Samoilova, E. V., Korotaeva, A. A., Rubanovich, A. V., and Kaminnyi, A. I. (2015) The association of PLA2G2A single nucleotide polymorphisms with type IIa secretory phospholipase A2 level but not its activity in patients with stable coronary heart disease. Gene 564, 29-34

24. Wootton, P. T., Drenos, F., Cooper, J. A., Thompson, S. R., Stephens, J. W., Hurt-Camejo, E., Wiklund, O., Humphries, S. E., and Talmud, P. J. (2006) 
Tagging-SNP haplotype analysis of the secretory PLA2IIa gene PLA2G2A shows strong association with serum levels of sPLA2IIa: results from the UDACS study. Human molecular genetics 15, 355-361

25. Qu, X. D., and Lehrer, R. I. (1998) Secretory phospholipase A2 is the principal bactericide for staphylococci and other gram-positive bacteria in human tears. Infection and immunity 66, 2791-2797

26. Harwig, S. S., Tan, L., Qu, X. D., Cho, Y., Eisenhauer, P. B., and Lehrer, R. I. (1995) Bactericidal properties of murine intestinal phospholipase A2. The Journal of clinical investigation 95, 603-610

27. Weinrauch, Y., Elsbach, P., Madsen, L. M., Foreman, A., and Weiss, J. (1996) The potent anti-Staphylococcus aureus activity of a sterile rabbit inflammatory fluid is due to a $14-\mathrm{kD}$ phospholipase A2. The Journal of clinical investigation 97, 250-257

28. Mansbach, C. M., 2nd, Pieroni, G., and Verger, R. (1982) Intestinal phospholipase, a novel enzyme. The Journal of clinical investigation 69, 368-376

29. Verger, R., Ferrato, F., Mansbach, C. M., and Pieroni, G. (1982) Novel intestinal phospholipase A2: purification and some molecular characteristics. Biochemistry 21, 6883-6889

30. Augert, A., Payre, C., de Launoit, Y., Gil, J., Lambeau, G., and Bernard, D. (2009) The M-type receptor PLA2R regulates senescence through the p53 pathway. EMBO reports 10, 271-277

31. Ganesan, K., Ivanova, T., Wu, Y., Rajasegaran, V., Wu, J., Lee, M. H., Yu, K., Rha, S. Y., Chung, H. C., Ylstra, B., Meijer, G., Lian, K. O., Grabsch, H., and Tan, P. (2008) Inhibition of gastric cancer invasion and metastasis by PLA2G2A, a novel beta-catenin/TCF target gene. Cancer research 68, 4277-4286

32. Leung, S. Y., Chen, X., Chu, K. M., Yuen, S. T., Mathy, J., Ji, J., Chan, A. S., Li, R., Law, S., Troyanskaya, O. G., Tu, I. P., Wong, J., So, S., Botstein, D., and Brown, P. O. (2002) Phospholipase A2 group IIA expression in gastric adenocarcinoma is associated with prolonged survival and less frequent metastasis. Proceedings of the National Academy of Sciences of the United States of America 99, 16203-16208

33. Hui, D. Y. (2018) Group 1B phospholipase A2 in metabolic and inflammatory disease modulation. Biochimica et Biophysica Acta (BBA) - Molecular and Cell Biology of Lipids

34. Hollie, N. I., and Hui, D. Y. (2011) Group 1B phospholipase A2 deficiency protects against diet-induced hyperlipidemia in mice. Journal of lipid research $\mathbf{5 2}$, 2005-2011

35. Hollie, N. I., Konaniah, E. S., Goodin, C., and Hui, D. Y. (2014) Group 1B Phospholipase A2 Inactivation Suppresses Atherosclerosis and Metabolic Diseases in LDL Receptor-deficient Mice. Atherosclerosis 234, 377-380

36. Hui, D. Y., Cope, M. J., Labonte, E. D., Chang, H. T., Shao, J., Goka, E., Abousalham, A., Charmot, D., and Buysse, J. (2009) The phospholipase A(2) inhibitor methyl indoxam suppresses diet-induced obesity and glucose intolerance in mice. British journal of pharmacology 157, 1263-1269

37. Cash, J. G., Kuhel, D. G., Goodin, C., and Hui, D. Y. (2011) Pancreatic acinar cell-specific over-expression of group 1B phospholipase A(2) exacerbates diet- 
induced obesity and insulin resistance in mice. International journal of obesity (2005) 35, 877-881

38. Labonté, E. D., Kirby, R. J., Schildmeyer, N. M., Cannon, A. M., Huggins, K. W., and Hui, D. Y. (2006) Group 1B Phospholipase A(2)-Mediated Lysophospholipid Absorption Directly Contributes to Postprandial Hyperglycemia. Diabetes 55, 935-941

39. Richmond, B. L., Boileau, A. C., Zheng, S., Huggins, K. W., Granholm, N. A., Tso, P., and Hui, D. Y. (2001) Compensatory phospholipid digestion is required for cholesterol absorption in pancreatic phospholipase A(2)-deficient mice. Gastroenterology 120, 1193-1202

40. Labonté, E. D., Pfluger, P. T., Cash, J. G., Kuhel, D. G., Roja, J. C., Magness, D. P., Jandacek, R. J., Tschöp, M. H., and Hui, D. Y. (2010) Postprandial lysophospholipid suppresses hepatic fatty acid oxidation: the molecular link between group 1B phospholipase $\mathrm{A}(2)$ and diet-induced obesity. The FASEB Journal 24, 2516-2524

41. Cash, J. G., Konaniah, E., Hegde, N., Kuhel, D. G., Watanabe, M., RomickRosendale, L., and Hui, D. Y. (2018) Therapeutic reduction of lysophospholipids in the digestive tract recapitulates the metabolic benefits of bariatric surgery and promotes diabetes remission. Mol Metab

42. Pruzanski, W., and Vadas, P. (1989) Phospholipase A2 and inflammation. Ann Rheum Dis 48, 962-963

43. ANTONIO, V., BROUILLET, A., JANVIER, B., MONNE, C., BEREZIAT, G., ANDREANI, M., and RAYMONDJEAN, M. (2002) Transcriptional regulation of the rat type IIA phospholipase A2 gene by cAMP and interleukin-1 $\beta$ in vascular smooth muscle cells: interplay of the CCAAT/enhancer binding protein (C/EBP), nuclear factor- $\mathrm{\kappa B}$ and Ets transcription factors. Biochemical Journal 368, 415-424

44. Crowl, R. M., Stoller, T. J., Conroy, R. R., and Stoner, C. R. (1991) Induction of phospholipase A2 gene expression in human hepatoma cells by mediators of the acute phase response. Journal of Biological Chemistry 266, 2647-2651

45. Massaad, C., Paradon, M., Jacques, C., Salvat, C., Bereziat, G., Berenbaum, F., and Olivier, J.-L. (2000) Induction of Secreted Type IIA Phospholipase A2 Gene Transcription by Interleukin-1 $\beta$ : ROLE OF C/EBP FACTORS. Journal of Biological Chemistry 275, 22686-22694

46. Sharma, P., Levesque, T., Boilard, E., and Park, E. A. (2014) Thyroid hormone status regulates the expression of secretory phospholipases. Biochemical and Biophysical Research Communications 444, 56-62

47. Yamamoto, K., Isogai, Y., Sato, H., Taketomi, Y., and Murakami, M. (2011) Secreted phospholipase A2, lipoprotein hydrolysis, and atherosclerosis: integration with lipidomics. Analytical and bioanalytical chemistry 400, 18291842

48. Sun, C. Q., Zhong, C. Y., Sun, W. W., Xiao, H., Zhu, P., Lin, Y. Z., Zhang, C. L., Gao, H., and Song, Z. Y. (2016) Elevated Type II Secretory Phospholipase A2 Increases the Risk of Early Atherosclerosis in Patients with Newly Diagnosed Metabolic Syndrome. Sci Rep 6, 34929

49. Monroy-Munoz, I. E., Angeles-Martinez, J., Posadas-Sanchez, R., VillarrealMolina, T., Alvarez-Leon, E., Flores-Dominguez, C., Cardoso-Saldana, G., 
Medina-Urrutia, A., Juarez-Rojas, J. G., Posadas-Romero, C., and Alarcon, G. V. (2017) PLA2G2A polymorphisms are associated with metabolic syndrome and type 2 diabetes mellitus. Results from the genetics of atherosclerotic disease Mexican study. Immunobiology 222, 967-972

50. Iyer, A., Lim, J., Poudyal, H., Reid, R. C., Suen, J. Y., Webster, J., Prins, J. B., Whitehead, J. P., Fairlie, D. P., and Brown, L. (2012) An Inhibitor of Phospholipase A(2) Group IIA Modulates Adipocyte Signaling and Protects Against Diet-Induced Metabolic Syndrome in Rats. Diabetes 61, 2320-2329

51. Kuefner, M. S., Pham, K., Redd, J. R., Stephenson, E. J., Harvey, I., Deng, X., Bridges, D., Boilard, E., Elam, M. B., and Park, E. A. (2017) Secretory phospholipase A2 group IIA modulates insulin sensitivity and metabolism. Journal of lipid research 58, 1822-1833

52. Kuefner, M. S., Deng, X., Stephenson, E. J., Pham, K., and Park, E. A. (2018) Secretory phospholipase A2 group IIA enhances the metabolic rate and increases glucose utilization in response to thyroid hormone. FASEB journal : official publication of the Federation of American Societies for Experimental Biology, fj201800711R

53. Miki, Y., Yamamoto, K., Taketomi, Y., Sato, H., Shimo, K., Kobayashi, T., Ishikawa, Y., Ishii, T., Nakanishi, H., Ikeda, K., Taguchi, R., Kabashima, K., Arita, M., Arai, H., Lambeau, G., Bollinger, J. M., Hara, S., Gelb, M. H., and Murakami, M. (2013) Lymphoid tissue phospholipase A(2) group IID resolves contact hypersensitivity by driving antiinflammatory lipid mediators. The Journal of Experimental Medicine 210, 1217-1234

54. Ishizaki, J., Suzuki, N., Higashino, K., Yokota, Y., Ono, T., Kawamoto, K., Fujii, N., Arita, H., and Hanasaki, K. (1999) Cloning and characterization of novel mouse and human secretory phospholipase A(2)s. The Journal of biological chemistry 274, 24973-24979

55. Shakhov, A. N., Rubtsov, A. V., Lyakhov, I. G., Tumanov, A. V., and Nedospasov, S. A. (2000) SPLASH (PLA2IID), a novel member of phospholipase A2 family, is associated with lymphotoxin deficiency. Genes and immunity 1, 191-199

56. Valentin, E., Koduri, R. S., Scimeca, J. C., Carle, G., Gelb, M. H., Lazdunski, M., and Lambeau, G. (1999) Cloning and recombinant expression of a novel mousesecreted phospholipase A2. The Journal of biological chemistry 274, 1915219160

57. Lalia, A. Z., and Lanza, I. R. (2016) Insulin-Sensitizing Effects of Omega-3 Fatty Acids: Lost in Translation? Nutrients 8, 329

58. Thota, R. N., Acharya, S. H., and Garg, M. L. (2019) Curcumin and/or omega-3 polyunsaturated fatty acids supplementation reduces insulin resistance and blood lipids in individuals with high risk of type 2 diabetes: a randomised controlled trial. Lipids in health and disease 18, 31

59. Kalupahana, N. S., Claycombe, K., Newman, S. J., Stewart, T., Siriwardhana, N., Matthan, N., Lichtenstein, A. H., and Moustaid-Moussa, N. (2010)

Eicosapentaenoic acid prevents and reverses insulin resistance in high-fat dietinduced obese mice via modulation of adipose tissue inflammation. The Journal of nutrition 140, 1915-1922 
60. Lanza, I. R., Blachnio-Zabielska, A., Johnson, M. L., Schimke, J. M., Jakaitis, D. R., Lebrasseur, N. K., Jensen, M. D., Sreekumaran Nair, K., and Zabielski, P. (2013) Influence of fish oil on skeletal muscle mitochondrial energetics and lipid metabolites during high-fat diet. American journal of physiology. Endocrinology and metabolism 304, E1391-1403

61. Lionetti, L., Mollica, M. P., Sica, R., Donizzetti, I., Gifuni, G., Pignalosa, A., Cavaliere, G., and Putti, R. (2014) Differential effects of high-fish oil and highlard diets on cells and cytokines involved in the inflammatory process in rat insulin-sensitive tissues. International journal of molecular sciences 15, 30403063

62. Liu, X., Xue, Y., Liu, C., Lou, Q., Wang, J., Yanagita, T., Xue, C., and Wang, Y. (2013) Eicosapentaenoic acid-enriched phospholipid ameliorates insulin resistance and lipid metabolism in diet-induced-obese mice. Lipids in health and disease 12, 109

63. de Castro, G. S., Deminice, R., Simoes-Ambrosio, L. M., Calder, P. C., Jordao, A. A., and Vannucchi, H. (2015) Dietary docosahexaenoic acid and eicosapentaenoic acid influence liver triacylglycerol and insulin resistance in rats fed a highfructose diet. Marine drugs 13, 1864-1881

64. Guo, X.-f., Li, X., Shi, M., and Li, D. (2017) n-3 Polyunsaturated Fatty Acids and Metabolic Syndrome Risk: A Meta-Analysis. Nutrients 9, 703

65. Claria, J., Dalli, J., Yacoubian, S., Gao, F., and Serhan, C. N. (2012) Resolvin D1 and resolvin D2 govern local inflammatory tone in obese fat. Journal of immunology (Baltimore, Md. : 1950) 189, 2597-2605

66. Hellmann, J., Tang, Y., Kosuri, M., Bhatnagar, A., and Spite, M. (2011) Resolvin D1 decreases adipose tissue macrophage accumulation and improves insulin sensitivity in obese-diabetic mice. The FASEB Journal 25, 2399-2407

67. Kowala, M. C., and Schoefl, G. I. (1986) The popliteal lymph node of the mouse: internal architecture, vascular distribution and lymphatic supply. Journal of anatomy 148, 25-46

68. Levene, A. (1982) A Colour Atlas of Thymus and Lymph Node Histopathology with Ultrastructure. Journal of the Royal Society of Medicine 75, 381-381

69. Sato, H., Taketomi, Y., Ushida, A., Isogai, Y., Kojima, T., Hirabayashi, T., Miki, Y., Yamamoto, K., Nishito, Y., Kobayashi, T., Ikeda, K., Taguchi, R., Hara, S., Ida, S., Miyamoto, Y., Watanabe, M., Baba, H., Miyata, K., Oike, Y., Gelb, Michael H., and Murakami, M. (2014) The Adipocyte-Inducible Secreted Phospholipases PLA2G5 and PLA2G2E Play Distinct Roles in Obesity. Cell metabolism 20, 119-132

70. Suzuki, N., Ishizaki, J., Yokota, Y., Higashino, K.-i., Ono, T., Ikeda, M., Fujii, N., Kawamoto, K., and Hanasaki, K. (2000) Structures, Enzymatic Properties, and Expression of Novel Human and Mouse Secretory Phospholipase A2s. Journal of Biological Chemistry 275, 5785-5793

71. Yamamoto, K., Miki, Y., Sato, H., Nishito, Y., Gelb, M. H., Taketomi, Y., and Murakami, M. (2016) Expression and Function of Group IIE Phospholipase A(2) in Mouse Skin. The Journal of biological chemistry 291, 15602-15613 
72. Yamamoto, T., and Ryan, R. O. (2007) Anionic phospholipids inhibit apolipoprotein E--low-density lipoprotein receptor interactions. Biochemical and biophysical research communications 354, 820-824

73. Zhi, H., Qu, L., Wu, F., Chen, L., and Tao, J. (2015) Group IIE secretory phospholipase A2 regulates lipolysis in adipocytes. Obesity (Silver Spring, Md.) 23, 760-768

74. Sato, H., Kato, R., Isogai, Y., Saka, G., Ohtsuki, M., Taketomi, Y., Yamamoto, K., Tsutsumi, K., Yamada, J., Masuda, S., Ishikawa, Y., Ishii, T., Kobayashi, T., Ikeda, K., Taguchi, R., Hatakeyama, S., Hara, S., Kudo, I., Itabe, H., and Murakami, M. (2008) Analyses of group III secreted phospholipase A2 transgenic mice reveal potential participation of this enzyme in plasma lipoprotein modification, macrophage foam cell formation, and atherosclerosis. The Journal of biological chemistry 283, 33483-33497

75. Hill, A. A., Bolus, W. R., and Hasty, A. H. (2014) A Decade of Progress in Adipose Tissue Macrophage Biology. Immunological reviews 262, 134-152

76. Odegaard, J. I., and Chawla, A. (2013) The immune system as a sensor of the metabolic state. Immunity 38, 644-654

77. Zhang, Q., Hao, H., Xie, Z., Cheng, Y., Yin, Y., Xie, M., Huang, H., Gao, J., Liu, H., Tong, C., Zang, L., Mu, Y., and Han, W. (2017) M2 macrophages infusion ameliorates obesity and insulin resistance by remodeling inflammatory/macrophages' homeostasis in obese mice. Molecular and Cellular Endocrinology 443, 63-71

78. Shridas, P., Noffsinger, V. P., Trumbauer, A. C., and Webb, N. R. (2017) The dual role of group $\mathrm{V}$ secretory phospholipase A2 in pancreatic beta-cells. Endocrine 58, 47-58

79. Ramanadham, S., Bohrer, A., Mueller, M., Jett, P., Gross, R. W., and Turk, J. (1993) Mass spectrometric identification and quantitation of arachidonatecontaining phospholipids in pancreatic islets: prominence of plasmenylethanolamine molecular species. Biochemistry 32, 5339-5351

80. Ramanadham, S., Hsu, F. F., Zhang, S., Jin, C., Bohrer, A., Song, H., Bao, S., Ma, Z., and Turk, J. (2004) Apoptosis of insulin-secreting cells induced by endoplasmic reticulum stress is amplified by overexpression of group VIA calcium-independent phospholipase A2 (iPLA2 beta) and suppressed by inhibition of iPLA2 beta. Biochemistry 43, 918-930

81. Robertson, R. P., Tsai, P., Little, S. A., Zhang, H. J., and Walseth, T. F. (1987) Receptor-mediated adenylate cyclase-coupled mechanism for PGE2 inhibition of insulin secretion in HIT cells. Diabetes 36, 1047-1053

82. Smyth, E. M., Grosser, T., Wang, M., Yu, Y., and FitzGerald, G. A. (2009) Prostanoids in health and disease. Journal of lipid research 50 Suppl, S423-428

83. Yajima, H., Komatsu, M., Schermerhorn, T., Aizawa, T., Kaneko, T., Nagai, M., Sharp, G. W., and Hashizume, K. (1999) cAMP enhances insulin secretion by an action on the ATP-sensitive $\mathrm{K}+$ channel-independent pathway of glucose signaling in rat pancreatic islets. Diabetes 48, 1006-1012

84. Li, X., Shridas, P., Forrest, K., Bailey, W., and Webb, N. R. (2010) Group X secretory phospholipase $\mathrm{A}(2)$ negatively regulates adipogenesis in murine models. The FASEB Journal 24, 4313-4324 
85. Shridas, P., Bailey, W. M., Boyanovsky, B. B., Oslund, R. C., Gelb, M. H., and Webb, N. R. (2010) Group X Secretory Phospholipase A2 Regulates the Expression of Steroidogenic Acute Regulatory Protein (StAR) in Mouse Adrenal Glands. Journal of Biological Chemistry 285, 20031-20039

86. Cupillard, L., Koumanov, K., Mattei, M. G., Lazdunski, M., and Lambeau, G. (1997) Cloning, chromosomal mapping, and expression of a novel human secretory phospholipase A2. The Journal of biological chemistry 272, 1574515752

87. Matthias, A., Ohlson, K. B. E., Fredriksson, J. M., Jacobsson, A., Nedergaard, J., and Cannon, B. (2000) Thermogenic Responses in Brown Fat Cells Are Fully UCP1-dependent: UCP2 OR UCP3 DO NOT SUBSTITUTE FOR UCP1 IN ADRENERGICALLY OR FATTY ACID-INDUCED THERMOGENESIS. Journal of Biological Chemistry 275, 25073-25081

88. Morioka, Y., Saiga, A., Yokota, Y., Suzuki, N., Ikeda, M., Ono, T., Nakano, K., Fujii, N., Ishizaki, J., Arita, H., and Hanasaki, K. (2000) Mouse Group X

Secretory Phospholipase A2 Induces a Potent Release of Arachidonic Acid from Spleen Cells and Acts as a Ligand for the Phospholipase A2 Receptor. Archives of Biochemistry and Biophysics 381, 31-42

89. Sato, H., Isogai, Y., Masuda, S., Taketomi, Y., Miki, Y., Kamei, D., Hara, S., Kobayashi, T., Ishikawa, Y., Ishii, T., Ikeda, K., Taguchi, R., Ishimoto, Y., Suzuki, N., Yokota, Y., Hanasaki, K., Suzuki-Yamamoto, T., Yamamoto, K., and Murakami, M. (2011) Physiological Roles of Group X-secreted Phospholipase A2 in Reproduction, Gastrointestinal Phospholipid Digestion, and Neuronal Function. Journal of Biological Chemistry 286, 11632-11648

90. Chu, K., Miyazaki, M., Man, W. C., and Ntambi, J. M. (2006) Stearoyl-coenzyme A desaturase 1 deficiency protects against hypertriglyceridemia and increases plasma high-density lipoprotein cholesterol induced by liver X receptor activation. Molecular and cellular biology 26, 6786-6798

91. Joseph, S. B., Laffitte, B. A., Patel, P. H., Watson, M. A., Matsukuma, K. E., Walczak, R., Collins, J. L., Osborne, T. F., and Tontonoz, P. (2002) Direct and indirect mechanisms for regulation of fatty acid synthase gene expression by liver $\mathrm{X}$ receptors. The Journal of biological chemistry 277, 11019-11025

92. Repa, J. J., Liang, G., Ou, J., Bashmakov, Y., Lobaccaro, J. M., Shimomura, I., Shan, B., Brown, M. S., Goldstein, J. L., and Mangelsdorf, D. J. (2000)

Regulation of mouse sterol regulatory element-binding protein-1c gene (SREBP1c) by oxysterol receptors, LXRalpha and LXRbeta. Genes \& development 14, 2819-2830

93. Asensio, C., Muzzin, P., and Rohner-Jeanrenaud, F. (2004) Role of glucocorticoids in the physiopathology of excessive fat deposition and insulin resistance. International journal of obesity and related metabolic disorders : journal of the International Association for the Study of Obesity 28 Suppl 4, S4552

94. Pivonello, R., De Leo, M., Vitale, P., Cozzolino, A., Simeoli, C., De Martino, M. C., Lombardi, G., and Colao, A. (2010) Pathophysiology of diabetes mellitus in Cushing's syndrome. Neuroendocrinology 92 Suppl 1, 77-81 
95. Stojanovska, L., Rosella, G., and Proietto, J. (1990) Evolution of dexamethasoneinduced insulin resistance in rats. The American journal of physiology 258, E748756

96. Zakrzewska, K. E., Cusin, I., Stricker-Krongrad, A., Boss, O., Ricquier, D., Jeanrenaud, B., and Rohner-Jeanrenaud, F. (1999) Induction of obesity and hyperleptinemia by central glucocorticoid infusion in the rat. Diabetes 48,365 370

97. Shridas, P., Zahoor, L., Forrest, K. J., Layne, J. D., and Webb, N. R. (2014) Group X secretory phospholipase A2 regulates insulin secretion through a cyclooxygenase-2-dependent mechanism. The Journal of biological chemistry 289, 27410-27417

98. Rouault, M., Bollinger, J. G., Lazdunski, M., Gelb, M. H., and Lambeau, G. (2003) Novel mammalian group XII secreted phospholipase A2 lacking enzymatic activity. Biochemistry 42, 11494-11503

99. Sladek, F. M., Zhong, W. M., Lai, E., and Darnell, J. E., Jr. (1990) Liver-enriched transcription factor HNF-4 is a novel member of the steroid hormone receptor superfamily. Genes \& development 4, 2353-2365

100. Guan, M., Qu, L., Tan, W., Chen, L., and Wong, C.-W. (2011) Hepatocyte nuclear factor-4 alpha regulates liver triglyceride metabolism in part through secreted phospholipase A2 GXIIB. Hepatology 53, 458-466

101. Aljakna, A., Choi, S., Savage, H., Hageman Blair, R., Gu, T., Svenson, K. L., Churchill, G. A., Hibbs, M., and Korstanje, R. (2012) Pla2g12b and Hpn Are Genes Identified by Mouse ENU Mutagenesis That Affect HDL Cholesterol. PloS one 7, e43139

102. Sheena, V., Hertz, R., Nousbeck, J., Berman, I., Magenheim, J., and Bar-Tana, J. (2005) Transcriptional regulation of human microsomal triglyceride transfer protein by hepatocyte nuclear factor-4 $\alpha$. Journal of lipid research 46, 328-341

103. Yin, L., Ma, H., Ge, X., Edwards, P. A., and Zhang, Y. (2011) Hepatic HNF4 $\alpha$ Is Essential for Maintaining Triglyceride and Cholesterol Homeostasis. Arteriosclerosis, thrombosis, and vascular biology 31, 328-336

104. Hayhurst, G. P., Lee, Y.-H., Lambert, G., Ward, J. M., and Gonzalez, F. J. (2001) Hepatocyte Nuclear Factor 4 $\alpha$ (Nuclear Receptor 2A1) Is Essential for Maintenance of Hepatic Gene Expression and Lipid Homeostasis. Molecular and Cellular Biology 21, 1393-1403

105. Liu, Q., Yang, M., Fu, X., Liu, R., Sun, C., Pan, H., Wong, C.-W., and Guan, M. (2016) Activation of farnesoid $X$ receptor promotes triglycerides lowering by suppressing phospholipase A2 G12B expression. Molecular and Cellular Endocrinology 436, 93-101

106. Ahn, S.-H., Shah, Y. M., Inoue, J., Morimura, K., Kim, I., Yim, S., Lambert, G., Kurotani, R., Nagashima, K., Gonzalez, F. J., and Inoue, Y. (2008) Hepatocyte nuclear factor $4 \alpha$ in the intestinal epithelial cells protects against inflammatory bowel disease. Inflammatory Bowel Diseases 14, 908-920

107. Fang, S., Suh, J. M., Reilly, S. M., Yu, E., Osborn, O., Lackey, D., Yoshihara, E., Perino, A., Jacinto, S., Lukasheva, Y., Atkins, A. R., Khvat, A., Schnabl, B., Yu, R. T., Brenner, D. A., Coulter, S., Liddle, C., Schoonjans, K., Olefsky, J. M., Saltiel, A. R., Downes, M., and Evans, R. M. (2015) Intestinal FXR agonism 
promotes adipose tissue browning and reduces obesity and insulin resistance. Nature Medicine 21, 159

108. Trabelsi, M. S., Daoudi, M., Prawitt, J., Ducastel, S., Touche, V., Sayin, S. I., Perino, A., Brighton, C. A., Sebti, Y., Kluza, J., Briand, O., Dehondt, H., Vallez, E., Dorchies, E., Baud, G., Spinelli, V., Hennuyer, N., Caron, S., Bantubungi, K., Caiazzo, R., Reimann, F., Marchetti, P., Lefebvre, P., Backhed, F., Gribble, F. M., Schoonjans, K., Pattou, F., Tailleux, A., Staels, B., and Lestavel, S. (2015)

Farnesoid X receptor inhibits glucagon-like peptide-1 production by enteroendocrine L cells. Nature communications 6, 7629

109. Lambeau, G., and Gelb, M. H. (2008) Biochemistry and physiology of mammalian secreted phospholipases A2. Annual review of biochemistry 77, 495520

110. Dennis, E. A., Cao, J., Hsu, Y. H., Magrioti, V., and Kokotos, G. (2011) Phospholipase A2 enzymes: physical structure, biological function, disease implication, chemical inhibition, and therapeutic intervention. Chemical reviews 111, 6130-6185

111. Kudo, I., and Murakami, M. (2002) Phospholipase A2 enzymes. Prostaglandins \& other lipid mediators 68-69, 3-58

112. Lu, B., Bridges, D., Yang, Y., Fisher, K., Cheng, A., Chang, L., Meng, Z. X., Lin, J. D., Downes, M., Yu, R. T., Liddle, C., Evans, R. M., and Saltiel, A. R. (2014) Metabolic crosstalk: molecular links between glycogen and lipid metabolism in obesity. Diabetes 63, 2935-2948

113. Stephenson, E. J., Ragauskas, A., Jaligama, S., Redd, J. R., Parvathareddy, J., Peloquin, M. J., Saravia, J., Han, J. C., Cormier, S. A., and Bridges, D. (2016) Exposure to environmentally persistent free radicals during gestation lowers energy expenditure and impairs skeletal muscle mitochondrial function in adult mice. American journal of physiology. Endocrinology and metabolism 310, E1003-1015

114. Sharma, P., Thakran, S., Deng, X., Elam, M. B., and Park, E. A. (2013) Nuclear Corepressors Mediate the Repression of Phospholipase A2 Group IIa Gene Transcription by Thyroid Hormone. Journal of Biological Chemistry 288, 1632116333

115. Attia, R. R., Sharma, P., Janssen, R. C., Friedman, J. E., Deng, X., Lee, J. S., Elam, M. B., Cook, G. A., and Park, E. A. (2011) Regulation of pyruvate dehydrogenase kinase 4 (PDK4) by CCAAT/enhancer-binding protein beta (C/EBPbeta). The Journal of biological chemistry 286, 23799-23807

116. Folch, J., Lees, M., and Sloane Stanley, G. H. (1957) A simple method for the isolation and purification of total lipides from animal tissues. The Journal of biological chemistry 226, 497-509

117. Murakami, M., Taketomi, Y., Girard, C., Yamamoto, K., and Lambeau, G. (2010) Emerging roles of secreted phospholipase A2 enzymes: Lessons from transgenic and knockout mice. Biochimie 92, 561-582

118. Breitling, L. P., Koenig, W., Fischer, M., Mallat, Z., Hengstenberg, C., Rothenbacher, D., and Brenner, H. (2011) Type II secretory phospholipase A2 and prognosis in patients with stable coronary heart disease: mendelian randomization study. PloS one 6, e22318 
119. Kajimura, S., Seale, P., Kubota, K., Lunsford, E., Frangioni, J. V., Gygi, S. P., and Spiegelman, B. M. (2009) Initiation of myoblast to brown fat switch by a PRDM16-C/EBP-beta transcriptional complex. Nature 460, 1154-1158

120. Puigserver, P., Wu, Z., Park, C. W., Graves, R., Wright, M., and Spiegelman, B. M. (1998) A cold-inducible coactivator of nuclear receptors linked to adaptive thermogenesis. Cell 92, 829-839

121. Seale, P., Kajimura, S., and Spiegelman, B. M. (2009) Transcriptional control of brown adipocyte development and physiological function--of mice and men. Genes \& development 23, 788-797

122. Echtay, K. S., Esteves, T. C., Pakay, J. L., Jekabsons, M. B., Lambert, A. J., Portero-Otin, M., Pamplona, R., Vidal-Puig, A. J., Wang, S., Roebuck, S. J., and Brand, M. D. (2003) A signalling role for 4-hydroxy-2-nonenal in regulation of mitochondrial uncoupling. The EMBO journal 22, 4103-4110

123. Reilly, M. P., Lawson, J. A., and FitzGerald, G. A. (1998) Eicosanoids and isoeicosanoids: indices of cellular function and oxidant stress. The Journal of nutrition 128, 434S-438S

124. Bertholet, A. M., Kazak, L., Chouchani, E. T., Bogaczynska, M. G., Paranjpe, I., Wainwright, G. L., Betourne, A., Kajimura, S., Spiegelman, B. M., and Kirichok, Y. (2017) Mitochondrial Patch Clamp of Beige Adipocytes Reveals UCP1Positive and UCP1-Negative Cells Both Exhibiting Futile Creatine Cycling. Cell metabolism 25, 811-822.e814

125. Fedorenko, A., Lishko, P. V., and Kirichok, Y. (2012) Mechanism of fatty-aciddependent UCP1 uncoupling in brown fat mitochondria. Cell 151, 400-413

126. Biddinger, S. B., Hernandez-Ono, A., Rask-Madsen, C., Haas, J. T., Aleman, J. O., Suzuki, R., Scapa, E. F., Agarwal, C., Carey, M. C., Stephanopoulos, G., Cohen, D. E., King, G. L., Ginsberg, H. N., and Kahn, C. R. (2008) Hepatic insulin resistance is sufficient to produce dyslipidemia and susceptibility to atherosclerosis. Cell metabolism 7, 125-134

127. Samuel, V. T., and Shulman, G. I. (2012) Mechanisms for insulin resistance: common threads and missing links. Cell 148, 852-871

128. Huggins, K. W., Boileau, A. C., and Hui, D. Y. (2002) Protection against dietinduced obesity and obesity- related insulin resistance in Group 1B PLA2deficient mice. American journal of physiology. Endocrinology and metabolism 283, E994-e1001

129. Hollie, N. I., Cash, J. G., Matlib, M. A., Wortman, M., Basford, J. E., Abplanalp, W., and Hui, D. Y. (2014) Micromolar changes in lysophosphatidylcholine concentration cause minor effects on mitochondrial permeability but major alterations in function. Biochimica et biophysica acta 1841, 888-895

130. Cypess, A. M., and Kahn, C. R. (2010) Brown fat as a therapy for obesity and diabetes. Current opinion in endocrinology, diabetes, and obesity 17, 143-149

131. Spiegelman, B. M., and Flier, J. S. (2001) Obesity and the regulation of energy balance. Cell 104, 531-543

132. Bartelt, A., Bruns, O. T., Reimer, R., Hohenberg, H., Ittrich, H., Peldschus, K., Kaul, M. G., Tromsdorf, U. I., Weller, H., Waurisch, C., Eychmuller, A., Gordts, P. L., Rinninger, F., Bruegelmann, K., Freund, B., Nielsen, P., Merkel, M., and 
Heeren, J. (2011) Brown adipose tissue activity controls triglyceride clearance. Nat Med 17, 200-205

133. Stanford, K. I., Middelbeek, R. J., Townsend, K. L., An, D., Nygaard, E. B., Hitchcox, K. M., Markan, K. R., Nakano, K., Hirshman, M. F., Tseng, Y. H., and Goodyear, L. J. (2013) Brown adipose tissue regulates glucose homeostasis and insulin sensitivity. The Journal of clinical investigation 123, 215-223

134. Stine, R. R., Shapira, S. N., Lim, H. W., Ishibashi, J., Harms, M., Won, K. J., and Seale, P. (2016) EBF2 promotes the recruitment of beige adipocytes in white adipose tissue. Mol Metab 5, 57-65

135. Chakraborty, D., Bhattacharya, A., and Mittal, B. R. (2015) Patterns of brown fat uptake of 18F-fluorodeoxyglucose in positron emission tomography/computed tomography scan. Indian journal of nuclear medicine : IJNM : the official journal of the Society of Nuclear Medicine, India 30, 320-322

136. Brent, G. A. (2012) Mechanisms of thyroid hormone action. The Journal of clinical investigation 122, 3035-3043

137. Oetting, A., and Yen, P. M. (2007) New insights into thyroid hormone action. Best practice \& research. Clinical endocrinology \& metabolism 21, 193-208

138. Mullur, R., Liu, Y.-Y,. \& Brent, G. A. . (2014) Thyroid Hormone Regulation of Metabolism. Physiological Reviews 94, 355-382

139. Weiner, J., Kranz, M., Klöting, N., Kunath, A., Steinhoff, K., Rijntjes, E., Köhrle, J., Zeisig, V., Hankir, M., Gebhardt, C., Deuther-Conrad, W., Heiker, J. T., Kralisch, S., Stumvoll, M., Blüher, M., Sabri, O., Hesse, S., Brust, P., Tönjes, A., and Krause, K. (2016) Thyroid hormone status defines brown adipose tissue activity and browning of white adipose tissues in mice. Scientific Reports $\mathbf{6}$, 38124

140. Martínez-Sánchez, N., Moreno-Navarrete, J. M., Contreras, C., Rial-Pensado, E., Fernø, J., Nogueiras, R., Diéguez, C., Fernández-Real, J.-M., and López, M. (2016) Thyroid hormones induce browning of white fat. The Journal of Endocrinology 232, 351-362

141. López, M., Alvarez, C. V., Nogueiras, R., and Diéguez, C. (2013) Energy balance regulation by thyroid hormones at central level. Trends in Molecular Medicine 19, 418-427

142. Warner, A., and Mittag, J. (2012) Thyroid hormone and the central control of homeostasis. Journal of molecular endocrinology 49, R29-35

143. Sato, H., Taketomi, Y., and Murakami, M. (2016) Metabolic regulation by secreted phospholipase A2. Inflamm Regen 36, 7

144. Bianco, A. C., Anderson, G., Forrest, D., Galton, V. A., Gereben, B., Kim, B. W., Kopp, P. A., Liao, X. H., Obregon, M. J., Peeters, R. P., Refetoff, S., Sharlin, D. S., Simonides, W. S., Weiss, R. E., and Williams, G. R. (2014) American Thyroid Association Guide to investigating thyroid hormone economy and action in rodent and cell models. Thyroid : official journal of the American Thyroid Association 24, 88-168

145. Peronnet, F., and Massicotte, D. (1991) Table of nonprotein respiratory quotient: an update. Canadian journal of sport sciences $=$ Journal canadien des sciences du sport 16, 23-29 
146. Liisberg Aune, U., Ruiz, L., Kajimura, S. . (2013) Isolation and Differentation of Stromal Vascular Cells to Beige/Brite Cells. J. Vis. Exp. 73, e50191

147. Reed, B. C., and Lane, M. D. (1980) Insulin receptor synthesis and turnover in differentiating 3T3-L1 preadipocytes. Proceedings of the National Academy of Sciences of the United States of America 77, 285-289

148. Harms, M. J., Ishibashi, J., Wang, W., Lim, H.-W., Goyama, S., Sato, T., Kurokawa, M., Won, K.-J., and Seale, P. (2014) Prdm16 is required for the maintenance of brown adipocyte identity and function in adult mice. Cell metabolism 19, 593-604

149. Noriko Suzuki, J. I., Yasunori Yokota, Ken-ichi Higashino, Takashi Ono, Minoru Ikeda, Noriko Fujii, Keiko Kawamoto and Kohji Hanasaki. (2000) Structures, Enzymatic Properties, and Expression of Novel Human and Mouse Secretory Phospholipase A2s. The Journal of biological chemistry 275, 5785-5793

150. Brent, G. A. (2012) Hypothyroidism and thyroiditis. In Williams Textbook of Endocrinology (Melmed SP, L. P., Kronenberg HM, ed), Philadelphia, PA

151. McAninch, E. A., and Bianco, A. C. (2014) Thyroid hormone signaling in energy homeostasis and energy metabolism. Annals of the New York Academy of Sciences 1311, 77-87

152. Andrew M. Shore, A. K., Paul Kemp, John R. Speakman, Neil S. Graham, Michael A. Lomax. (2013) Cold-Induced Changes in Gene Expression in Brown Adipose Tissue, White Adipose Tissue and Liver. PloS one 8, e68933

153. Cannon, B., and Nedergaard, J. (2004) Brown Adipose Tissue: Function and Physiological Significance. Physiological Reviews 84, 277-359

154. van der Lans, A. A., Hoeks, J., Brans, B., Vijgen, G. H., Visser, M. G., Vosselman, M. J., Hansen, J., Jorgensen, J. A., Wu, J., Mottaghy, F. M., Schrauwen, P., and van Marken Lichtenbelt, W. D. (2013) Cold acclimation recruits human brown fat and increases nonshivering thermogenesis. The Journal of clinical investigation 123, 3395-3403

155. Hope, P. J., Pyle, D., Daniels, C. B., Chapman, I., Horowitz, M., Morley, J. E., Trayhurn, P., Kumaratilake, J., and Wittert, G. (1997) Identification of brown fat and mechanisms for energy balance in the marsupial, Sminthopsis crassicaudata. The American journal of physiology 273, R161-167

156. James, D. E., Strube, M., and Muecdler, M. (1989) Molecular cloning and characterization of an insulin-regulatable glucose transporter. Nature 338, 83-87

157. Shimizu, Y., and Shimazu, T. (2002) Thyroid Hormone Augments GLUT4 Expression and Insulin-Sensitive Glucose Transport System in Differentiating Rat Brown Adipocytes in Culture. Journal of Veterinary Medical Science 64, 677-681

158. Lahesmaa, M., Orava, J., Schalin-Jantti, C., Soinio, M., Hannukainen, J. C., Noponen, T., Kirjavainen, A., Iida, H., Kudomi, N., Enerback, S., Virtanen, K. A., and Nuutila, P. (2014) Hyperthyroidism increases brown fat metabolism in humans. The Journal of clinical endocrinology and metabolism 99, E28-35

159. Wang, W., and Seale, P. (2016) Control of brown and beige fat development. Nature Reviews Molecular Cell Biology 17, 691-702

160. Rajakumari, S., Wu, J., Ishibashi, J., Hee-Woong, L., Giang, A.-H., Won, K.-J., Reed, R. R., and Seale, P. (2013) EBF2 determines and maintains brown adipocyte identity. Cell metabolism 17, 562-574 
161. Coe, N. R., Smith, A. J., Frohnert, B. I., Watkins, P. A., and Bernlohr, D. A. (1999) The Fatty Acid Transport Protein (FATP1) Is a Very Long Chain AcylCoA Synthetase. Journal of Biological Chemistry 274, 36300-36304

162. Wu, Q., Kazantzis, M., Doege, H., Ortegon, A. M., Tsang, B., Falcon, A., and Stahl, A. (2006) Fatty Acid Transport Protein 1 Is Required for Nonshivering Thermogenesis in Brown Adipose Tissue. Diabetes 55, 3229-3237

163. Thyagarajan, B., and Foster, M. T. (2017) Beiging of white adipose tissue as a therapeutic strategy for weight loss in humans. Hormone molecular biology and clinical investigation 31, 1868-1883

164. Seale, P., Conroe, H. M., Estall, J., Kajimura, S., Frontini, A., Ishibashi, J., Cohen, P., Cinti, S., and Spiegelman, B. M. (2011) Prdm16 determines the thermogenic program of subcutaneous white adipose tissue in mice. The Journal of clinical investigation 121, 96-105

165. Fleury, C., Neverova, M., Collins, S., Raimbault, S., Champigny, O., LeviMeyrueis, C., Bouillaud, F., Seldin, M. F., Surwit, R. S., Ricquier, D., and Warden, C. H. (1997) Uncoupling protein-2: a novel gene linked to obesity and hyperinsulinemia. Nat Genet 15, 269-272

166. Lanni, A., De Felice, M., Lombardi, A., Moreno, M., Fleury, C., Ricquier, D., and Goglia, F. (1997) Induction of UCP2 mRNA by thyroid hormones in rat heart. FEBS letters 418, 171-174

167. Chavin, K. D., Yang, S., Lin, H. Z., Chatham, J., Chacko, V. P., Hoek, J. B., Walajtys-Rode, E., Rashid, A., Chen, C. H., Huang, C. C., Wu, T. C., Lane, M. D., and Diehl, A. M. (1999) Obesity induces expression of uncoupling protein-2 in hepatocytes and promotes liver ATP depletion. The Journal of biological chemistry 274, 5692-5700

168. Purushotham, A., Schug, T. T., Xu, Q., Surapureddi, S., Guo, X., and Li, X. (2009) Hepatocyte-specific Deletion of SIRT1 Alters Fatty Acid Metabolism and Results in Hepatic Steatosis and Inflammation. Cell metabolism 9, 327-338

169. Saito, M., Okamatsu-Ogura, Y., Matsushita, M., Watanabe, K., Yoneshiro, T., Nio-Kobayashi, J., Iwanaga, T., Miyagawa, M., Kameya, T., Nakada, K., Kawai, Y., and Tsujisaki, M. (2009) High Incidence of Metabolically Active Brown Adipose Tissue in Healthy Adult Humans. Effects of Cold Exposure and Adiposity 58, 1526-1531

170. Kim, S. H., and Plutzky, J. (2016) Brown Fat and Browning for the Treatment of Obesity and Related Metabolic Disorders. Diabetes \& metabolism journal 40, 12 21

171. Poher, A.-L., Altirriba, J., Veyrat-Durebex, C., and Rohner-Jeanrenaud, F. (2015) Brown adipose tissue activity as a target for the treatment of obesity/insulin resistance. Frontiers in Physiology 6

172. Nicholls, D. G., and Locke, R. M. (1984) Thermogenic mechanisms in brown fat. Physiol Rev 64, 1-64

173. Ricquier, D. (2017) UCP1, the mitochondrial uncoupling protein of brown adipocyte: A personal contribution and a historical perspective. Biochimie 134, 38

174. García-Alonso, V., López-Vicario, C., Titos, E., Morán-Salvador, E., GonzálezPériz, A., Rius, B., Párrizas, M., Werz, O., Arroyo, V., and Clària, J. (2013) 
Coordinate Functional Regulation between Microsomal Prostaglandin E Synthase-1 (mPGES-1) and Peroxisome Proliferator-activated Receptor $\gamma$ (PPAR $\gamma$ ) in the Conversion of White-to-brown Adipocytes. Journal of Biological Chemistry 288, 28230-28242

175. Gnad, T., Scheibler, S., von Kügelgen, I., Scheele, C., Kilić, A., Glöde, A., Hoffmann, L. S., Reverte-Salisa, L., Horn, P., Mutlu, S., El-Tayeb, A., Kranz, M., Deuther-Conrad, W., Brust, P., Lidell, M. E., Betz, M. J., Enerbäck, S., Schrader, J., Yegutkin, G. G., Müller, C. E., and Pfeifer, A. (2014) Adenosine activates brown adipose tissue and recruits beige adipocytes via A2A receptors. Nature 516, 395

176. Lynes, M. D., Leiria, L. O., Lundh, M., Bartelt, A., Shamsi, F., Huang, T. L., Takahashi, H., Hirshman, M. F., Schlein, C., Lee, A., Baer, L. A., May, F. J., Gao, F., Narain, N. R., Chen, E. Y., Kiebish, M. A., Cypess, A. M., Blüher, M., Goodyear, L. J., Hotamisligil, G. S., Stanford, K. I., and Tseng, Y.-H. (2017) The cold-induced lipokine 12,13-diHOME promotes fatty acid transport into brown adipose tissue. Nature Medicine 23, 631

177. Larsen, S., Nielsen, J., Hansen, C. N., Nielsen, L. B., Wibrand, F., Stride, N., Schroder, H. D., Boushel, R., Helge, J. W., Dela, F., and Hey-Mogensen, M. (2012) Biomarkers of mitochondrial content in skeletal muscle of healthy young human subjects. The Journal of physiology 590, 3349-3360

178. Daval, M., Foufelle, F., and Ferre, P. (2006) Functions of AMP-activated protein kinase in adipose tissue. The Journal of physiology 574, 55-62

179. Wu, L., Zhang, L., Li, B., Jiang, H., Duan, Y., Xie, Z., Shuai, L., Li, J., and Li, J. (2018) AMP-Activated Protein Kinase (AMPK) Regulates Energy Metabolism through Modulating Thermogenesis in Adipose Tissue. Front Physiol 9, 122

180. Anderson, C. M., and Stahl, A. (2013) SLC27 fatty acid transport proteins. Molecular aspects of medicine 34, 516-528

181. Palmieri, F. (2013) The mitochondrial transporter family SLC25: identification, properties and physiopathology. Mol Aspects Med 34, 465-484

182. Cypess, A. M., and Kahn, C. R. (2010) The role and importance of brown adipose tissue in energy homeostasis. Current opinion in pediatrics 22, 478-484

183. Ricquier, D., Mory, G., and Hemon, P. (1979) Changes induced by cold adaptation in the brown adipose tissue from several species of rodents, with special reference to the mitochondrial components. Canadian journal of biochemistry 57, 1262-1266

184. Ueta, C. B., Fernandes, G. W., Capelo, L. P., Fonseca, T. L., Maculan, F. D. A., Gouveia, C. H. A., Brum, P. C., Christoffolete, M. A., Aoki, M. S., Lancellotti, C. L., Kim, B., Bianco, A. C., and Ribeiro, M. O. (2012) $\beta(1)$ Adrenergic receptor is key to cold- and diet-induced thermogenesis in mice. The Journal of endocrinology 214, 359-365

185. Cui, X., Nguyen, N. L., Zarebidaki, E., Cao, Q., Li, F., Zha, L., Bartness, T., Shi, H., and Xue, B. (2016) Thermoneutrality decreases thermogenic program and promotes adiposity in high-fat diet-fed mice. Physiological reports $\mathbf{4}$

186. Hibi, M., Oishi, S., Matsushita, M., Yoneshiro, T., Yamaguchi, T., Usui, C., Yasunaga, K., Katsuragi, Y., Kubota, K., Tanaka, S., and Saito, M. (2016) Brown adipose tissue is involved in diet-induced thermogenesis and whole-body fat 
utilization in healthy humans. International journal of obesity (2005) 40, 16551661

187. Gonzalez-Hurtado, E., Lee, J., Choi, J., and Wolfgang, M. J. (2017) Fatty acid oxidation is required for active and quiescent brown adipose tissue maintenance and thermogenic programing. Molecular metabolism 7, 45-56

188. Lee, J., Ellis, J. M., and Wolfgang, M. J. (2015) Adipose fatty acid oxidation is required for thermogenesis and potentiates oxidative stress-induced inflammation. Cell reports 10, 266-279

189. Ellis, J. M., Li, L. O., Wu, P. C., Koves, T. R., Ilkayeva, O., Stevens, R. D., Watkins, S. M., Muoio, D. M., and Coleman, R. A. (2010) Adipose acyl-CoA synthetase-1 directs fatty acids toward beta-oxidation and is required for cold thermogenesis. Cell metabolism 12, 53-64

190. Janssen, I., Heymsfield, S. B., Wang, Z. M., and Ross, R. (2000) Skeletal muscle mass and distribution in 468 men and women aged 18-88 yr. Journal of applied physiology (Bethesda, Md. : 1985) 89, 81-88

191. Zurlo, F., Larson, K., Bogardus, C., and Ravussin, E. (1990) Skeletal muscle metabolism is a major determinant of resting energy expenditure. The Journal of clinical investigation 86, 1423-1427

192. Zurlo, F., Nemeth, P. M., Choksi, R. M., Sesodia, S., and Ravussin, E. (1994) Whole-body energy metabolism and skeletal muscle biochemical characteristics. Metabolism: clinical and experimental 43, 481-486

193. Pant, M., Bal, N. C., and Periasamy, M. (2016) Sarcolipin: A Key Thermogenic and Metabolic Regulator in Skeletal Muscle. Trends in endocrinology and metabolism: TEM 27, 881-892

194. Periasamy, M., Maurya, S. K., Sahoo, S. K., Singh, S., Sahoo, S. K., Reis, F. C. G., and Bal, N. C. (2017) Role of SERCA Pump in Muscle Thermogenesis and Metabolism. Comprehensive Physiology 7, 879-890

195. Mineo, P. M., Cassell, E. A., Roberts, M. E., and Schaeffer, P. J. (2012) Chronic cold acclimation increases thermogenic capacity, non-shivering thermogenesis and muscle citrate synthase activity in both wild-type and brown adipose tissue deficient mice. Comparative biochemistry and physiology. Part A, Molecular \& integrative physiology 161, 395-400

196. Vigelso, A., Andersen, N. B., and Dela, F. (2014) The relationship between skeletal muscle mitochondrial citrate synthase activity and whole body oxygen uptake adaptations in response to exercise training. International journal of physiology, pathophysiology and pharmacology 6, 84-101

197. Calderon-Dominguez, M., Alcalá, M., Sebastián, D., Zorzano, A., Viana, M., Serra, D., and Herrero, L. (2017) Bioenergetics: Brown Adipose Tissue Bioenergetics: A New Methodological Approach (Adv. Sci. 4/2017). Advanced Science 4, n/a-n/a

198. Heaton, G. M., Wagenvoord, R. J., Kemp, A., Jr., and Nicholls, D. G. (1978) Brown-adipose-tissue mitochondria: photoaffinity labelling of the regulatory site of energy dissipation. European journal of biochemistry 82, 515-521

199. Giralt, M., and Villarroya, F. (2013) White, Brown, Beige/Brite: Different Adipose Cells for Different Functions? Endocrinology 154, 2992-3000 
200. Guerra, C., Koza, R. A., Yamashita, H., Walsh, K., and Kozak, L. P. (1998) Emergence of brown adipocytes in white fat in mice is under genetic control. Effects on body weight and adiposity. The Journal of clinical investigation 102, 412-420

201. Xue, B., Rim, J. S., Hogan, J. C., Coulter, A. A., Koza, R. A., and Kozak, L. P. (2007) Genetic variability affects the development of brown adipocytes in white fat but not in interscapular brown fat. Journal of lipid research 48, 41-51

202. Held, N. M., Kuipers, E. N., van Weeghel, M., van Klinken, J. B., Denis, S. W., Lombès, M., Wanders, R. J., Vaz, F. M., Rensen, P. C. N., Verhoeven, A. J., Boon, M. R., and Houtkooper, R. H. (2018) Pyruvate dehydrogenase complex plays a central role in brown adipocyte energy expenditure and fuel utilization during short-term beta-adrenergic activation. Scientific Reports 8, 9562

203. Weir, G., Ramage, L. E., Akyol, M., Rhodes, J. K., Kyle, C. J., Fletcher, A. M., Craven, T. H., Wakelin, S. J., Drake, A. J., Gregoriades, M. L., Ashton, C., Weir, N., van Beek, E. J. R., Karpe, F., Walker, B. R., and Stimson, R. H. (2018) Substantial Metabolic Activity of Human Brown Adipose Tissue during Warm Conditions and Cold-Induced Lipolysis of Local Triglycerides. Cell metabolism 27, 1348-1355.e1344

204. Yu, X. X., Lewin, D. A., Forrest, W., and Adams, S. H. (2002) Cold elicits the simultaneous induction of fatty acid synthesis and beta-oxidation in murine brown adipose tissue: prediction from differential gene expression and confirmation in vivo. FASEB journal : official publication of the Federation of American Societies for Experimental Biology 16, 155-168

205. Koepsell, H. (2013) The SLC22 family with transporters of organic cations, anions and zwitterions. Mol Aspects Med 34, 413-435

206. Exeter, H. J., Folkersen, L., Palmen, J., Franco-Cereceda, A., Cooper, J. A., Kalea, A. Z., Hooft, F. V., Eriksson, P., Humphries, S. E., and Talmud, P. J. (2012) Functional analysis of two PLA2G2A variants associated with secretory phospholipase A2-IIA levels. PloS one 7, e41139

207. Fitzgibbons, T. P., Kogan, S., Aouadi, M., Hendricks, G. M., Straubhaar, J., and Czech, M. P. (2011) Similarity of mouse perivascular and brown adipose tissues and their resistance to diet-induced inflammation. American journal of physiology. Heart and circulatory physiology 301, H1425-1437

208. Herrero, L., Shapiro, H., Nayer, A., Lee, J., and Shoelson, S. E. (2010) Inflammation and adipose tissue macrophages in lipodystrophic mice. Proceedings of the National Academy of Sciences of the United States of America 107, 240-245

209. Bae, J., Ricciardi, C. J., Esposito, D., Komarnytsky, S., Hu, P., Curry, B. J., Brown, P. L., Gao, Z., Biggerstaff, J. P., Chen, J., and Zhao, L. (2014) Activation of pattern recognition receptors in brown adipocytes induces inflammation and suppresses uncoupling protein 1 expression and mitochondrial respiration. American journal of physiology. Cell physiology 306, C918-930

210. Lorenzo, M., Fernandez-Veledo, S., Vila-Bedmar, R., Garcia-Guerra, L., De Alvaro, C., and Nieto-Vazquez, I. (2008) Insulin resistance induced by tumor necrosis factor-alpha in myocytes and brown adipocytes. Journal of animal science 86, E94-104 
211. Tourniaire, F., Romier-Crouzet, B., Lee, J. H., Marcotorchino, J., Gouranton, E., Salles, J., Malezet, C., Astier, J., Darmon, P., Blouin, E., Walrand, S., Ye, J., and Landrier, J.-F. (2013) Chemokine Expression in Inflamed Adipose Tissue Is Mainly Mediated by NF-кB. PloS one 8, e66515-e66515 


\section{VITA}

Michael Kuefner was born in Chicago, Illinois in 1993. In 2015, he graduated from Rhodes College in Memphis, Tennessee with a bachelor's in Biochemistry and Molecular Biology. In 2015 he joined the Biomedical Sciences program in the College of Graduate Health Sciences (CGHS) of University of Tennessee Health Science Center (UTHSC), working primarily in the areas of obesity and metabolism. He is expected to receive his Ph.D degree in 2019. 